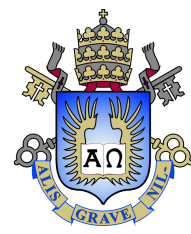

Jackes Martins da Silva

\title{
lgualdade de Jarzynski e troca de informação em sistemas não Markovianos
}

Tese de Doutorado

Tese apresentada como requisito parcial para obtenção do grau de Doutor em Física pelo Programa de Pós-graduação em Física, do Departamento de Física da PUC-Rio.

Orientador: Prof. Welles Antônio Martinez Morgado 

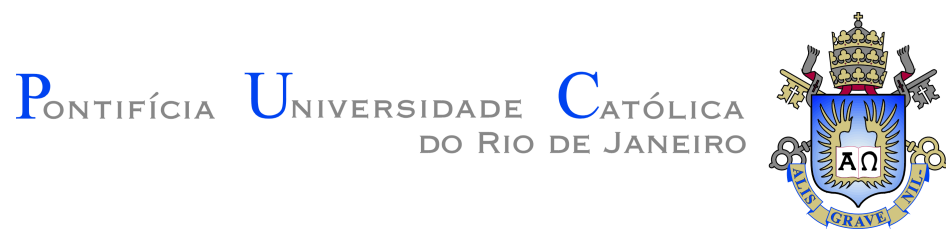

\section{Jackes Martins da Silva}

\section{Igualdade de Jarzynski e troca de informação em sistemas não Markovianos}

Tese apresentada como requisito parcial para obtenção do grau de Doutor em Física pelo Programa de Pós-graduação em Física, do Departamento de Física da PUC-Rio. Aprovada pela Comissão Examinadora abaixo:

Prof. Welles Antônio Martinez Morgado Orientador

Departamento de Física - PUC-Rio

Prof. Luca Roberto Augusto Moriconi

UFRJ

Prof. Rodrigo Barbosa Capaz

UFRJ

Prof. Jefferson Ferraz Damasceno Felix Araújo

Departamento de Física - PUC-Rio

Prof. Thiago Barbosa dos Santos Guerreiro

Departamento de Física - PUC-Rio

Prof. Wei Chen

Departamento de Física - PUC-Rio

Profa. Celia Beatriz Anteneodo de Porto Departamento de Física - PUC-Rio 
Todos os direitos reservados. É proibida a reprodução total ou parcial do trabalho sem autorização da universidade, do autor e do orientador.

\section{Jackes Martins da Silva}

Graduou-se em Física pela Universidade Estadual de Maringá (UEM). Obteve o grau de Mestre pelo Departamento de Física da Pontifícia Universidade Católica do Rio de Janeiro (PUCRio).

Ficha Catalográfica

Martins da Silva, Jackes

Igualdade de Jarzynski e troca de informação em sistemas não Markovianos / Jackes Martins da Silva; orientador: Welles Antônio Martinez Morgado. - Rio de janeiro: PUC-Rio, Departamento de Física, 2020.

v., 99 f: il. color. ; $30 \mathrm{~cm}$

Tese (doutorado) - Pontifícia Universidade Católica do Rio de Janeiro, Departamento de Física.

Inclui bibliografia

1. Física - Teses. 2. Entropia;. 3. Igualdade de Jarzynski;. 4. Não-Markoviano;. 5. Modelo estocástico. I. Morgado, Welles Antônio Martinez. II. Pontifícia Universidade Católica do Rio de Janeiro. Departamento de Física. III. Título. 


\section{Agradecimentos}

Agradeço, primeiramente, ao meu orientador, Welles Morgado, pelos ensinamentos, pela dedicação e paciência no período que se estendeu a produção desta tese.

Agradeço a minha família pelo apoio durante estes anos. Em especial, a minha mãe, Lenir Oliveira, aos meus irmãos, Ederson Martins e Eliane Martins, e a minha tia, Vanda Oliveira, pelo carinho e auxílio.

Agradeço a todos os professores do Departamento de Física da PUCRio pelos ensinamentos, em especial, aos professores Marco Cremona e Hiroshi Nunokawa que sempre me atenderam gentilmente.

Agradeço aos funcionários da PUC-Rio por toda ajuda e diligência, em especial, à Giza, à Márcia e ao Julinho, sempre solícitos quando precisei de auxílio.

Agradeço a todos meus amigos e colegas. Em especial, agradeço à Milena Belançon, pelo auxílio nas correções ortográficas, ao Fernando Calsavara, pela ajuda com as traduções e ao Rodrigo Cunha, pelo companheirismo, apoio e afeto, nos bons e maus momentos, que foram essenciais para me manter persistente neste projeto. Também não poderia deixar de citar nominalmente aqueles que sempre me apoiaram neste período: Pablo Portes, Nelson Gaia, Domitila Becker, Jefferson Neves, Jefferson Ferraz, Mateus Fernandes, Aline Oliveira, Leonora Calsavara, Murilo Martins, Luiz Garcia, Rafael Mufato, Allan Gonçalves, Leandro Antunes, Flávia Estrada, Débora Ferreira, Matheus Brandão e Rodrigo Thomas.

Agradeço às agências de fomento, CAPES e CNPq, pelo auxílio financeiro concedido, sem os quais este trabalho não poderia ser realizado.

Por fim, agradeço a todos aqueles que, de uma forma ou de outra, me ajudaram e, por ventura, eu não citei anteriormente.

O presente trabalho foi realizado com apoio da Coordenação de Aperfeiçoamento de Pessoal de Nível Superior - Brasil (CAPES) - Código de Financiamento 001. 


\section{Resumo}

Martins da Silva, Jackes; Morgado, Welles Antônio Martinez. Igualdade de Jarzynski e troca de informação em sistemas não Markovianos. Rio de Janeiro, 2020. 99p. Tese de Doutorado - Departamento de Física, Pontifícia Universidade Católica do Rio de Janeiro.

A Igualdade de Jarzynski (IJ) é um tipo especial de Teorema de Flutuação, de trabalho, que caracteriza sistemas termodinâmicos microscópicos fora do equilíbrio. A IJ pode ser usada como uma calibração de experimentos e simulações, o que nos permite estudar comportamentos não triviais da dinâmica desses sistemas. Um desses comportamentos é a troca de entropia e informação que o sistema realiza junto a um banho térmico de contato. Neste ensejo, modelamos via uma dinâmica não-Markoviana, i.e., uma dinâmica com memória, que leva a fluxos reversos de informação do reservatório para o sistema.

\section{Palavras-chave}

Entropia; Igualdade de Jarzynski; Não-Markoviano; Modelo estocástico 


\section{Abstract}

Martins da Silva, Jackes; Morgado, Welles Antônio Martinez (Advisor). Jarzynski equality and information exchange in nonMarkovian systems. Rio de Janeiro, 2020. 99p. Tese de doutorado

- Departamento de Física, Pontifícia Universidade Católica do Rio de Janeiro.

The Jarzynski Equality (JE) is a special kind of Fluctuation Theorem, of work, which characterizes non-equilibrium small thermodynamics systems. The JE can be used as gauge of experiments and simulations allowing us to study the non-trivial behaviours of these systems' dynamics. One of these behaviours is the entropy and information flow the system makes in contact with a thermal bath. In this framework, we modelled through a non-Markovian dynamic, i.e., with a memory effect, leading to reverse flows of information from the reservoir to the system.

\section{Keywords}

Entropy; Jarzynski Equality; Non-Markovian; Stochastic model; 


\section{Sumário}

1 Introdução 12

2 Igualdade de Jarzinsky $\quad 15$

2.1 Termodinâmica de sistemas microscópicos $\quad 15$

2.1.1 Termodinâmica macroscópica: energia livre de Helmholtz e trabalho 16

2.1.2 Termodinâmica microscópica: energia livre de Helmholtz e trabalho 17

2.2 Informação associada a flutuações fora do equilíbrio 22

2.3 Evolução estocástica e simetria microscópica 26

$\begin{array}{lll}2.4 & \text { Entropia e trabalho dissipado } & 31\end{array}$

2.5 Equivalência de ensembles 33

2.6 Características do modelo 34

3 Modelo Fourier-Laplace $\quad 36$

3.1 Definição do modelo para o cálculo da distribuição de probabilidade estacionária $\quad 36$

3.1.1 Considerações sobre o ruído colorido $\eta(t) \quad 38$

$\begin{array}{ll}3.1 .2 & \text { Irreversibilidade }(z \rightarrow 0)\end{array}$

3.1.3 Integrais relevantes 41

3.1.3.1 Contribuição de $B(s) B(s) \quad 41$

3.1.3.2 Contribuição de $D(s) D(s) \quad 42$

3.1.3.3 Contribuição de $B(s) D(s) \quad 42$

3.1.4 Distribuição de probabilidade para a posição e velocidade no equilíbrio termodinâmico 43

3.1.5 Resumo das estapas do método 43

3.2 Função geradora de probabilidade para o trabalho de um sistema microscópico fora do equilíbrio 44

3.2.1 Função geradora de probabilidade para o trabalho externo 45

$\begin{array}{ll}3.2 .2 & \text { Igualdade de Jarzynski }\end{array}$

4 Modelo não markoviano linear e não linear $\quad 50$

4.1 O modelo não linear $\quad 50$

4.1.1 Etapas do processo termodinâmico $\quad 53$

4.1.2 Cumulantes do trabalho $W \quad 54$

4.2 Caso linear $\left(k_{3}=0\right) \quad 55$

4.2.1 Primeiro cumulante $\left\langle\overline{I_{\theta}}\right\rangle \quad 58$

4.2.2 Segundo cumulante $\left\langle\overline{I_{\theta}^{2}}\right\rangle \quad 58$

4.2.3 Igualdade de Jarzynski: caso linear $\quad 59$

$\begin{array}{ll}\text { 4.2.4 Produção de entropia } & 60\end{array}$

$\begin{array}{ll}\text { 4.2.5 Oscilações da entropia } & 66\end{array}$

4.3 Análise numérica para o caso não linear $\left(k_{3} \neq 0\right)$

4.3.1 Análise do calor $\quad 71$

4.3.2 Análise do trabalho 73

$\begin{array}{ll}\text { 4.3.3 Análise da entropia } & 75\end{array}$

$\begin{array}{ll}4.4 \text { Estados de "almoço grátis" } & 76\end{array}$ 
Referências bibliográficas

$\begin{array}{lr}\text { A } & 89\end{array}$

A.1 Considerações sobre a hamiltoniana de interação $\quad 89$

B $\quad \mathbf{9 0}$

B.1 Derivação da expressão de $\tilde{x}(s)$ e $\tilde{v}(s) \quad 90$

B.2 A distribuição de equilíbrio para a posição $x \quad 90$

B.3 Cálculo da contribuição de $B(s) B(s) \quad 91$

B.4 Modelo não linear (correção até a ordem $\left.\mathcal{O}\left(k_{3}^{1}\right)\right) \quad 92$

B.5 Expressão dos termos $A_{i}$ do segundo cumulante 93

$\begin{array}{ll}C & 95\end{array}$

C.1 Representação de $x^{3}(t)$ na forma integral $\quad 95$

C.2 Transformada de Laplace para cada termo da Equação de Langevin 95

C.3 Transformada de Laplace do segundo cumulante do ruído Gaussiano 96

C.4 Comportamento dos $\kappa_{i}$ 


\section{Lista de figuras}

Figura 2.1 Os círculos enumerados representam as três partículas do sistema. As coordenada $z_{1}, z_{2}$ e $z_{3}$ são as distâncias de cada partícula até a parede fixa (retângulo na vertical), explicitada para $z_{1}$. O círculo preto é o parâmetro do trabalho. A posição $\lambda$ é manipulada externamente seguindo um protocolo. As molas representam as interações par-a-par entre as partículas. Os graus de liberdade do reservatório térmico não estão ilustrados.

Figura 2.2 Ilustração das trocas de energia entre o sistema de interesse e o sistema externo, e entre o sistema de interesse e o reservatório térmico.

Figura 2.3 Movimento do volume no espaço de fase. A densidade dos sistemas do ensemble na vizinhança de um ponto do espaço de fase não muda com o tempo.

Figura 2.4 Distribuição do trabalho $\rho(W)$ para um sistema microscópico sujeito a um processo irreversível. A região na qual $W<\Delta F$ é a chamada proibitiva

Figura 2.5 Uma analogia para a produção de trabalho: esticar e contrair um elástico de borracha. Se esticássemos a borracha, a temperatura e a tensão seriam maiores do que no processo reverso, a contração. É um exemplo de ciclo de histerese no qual $W>0$.

Figura 2.6 Trajetória da contração (azul) e expansão (laranja) de uma sequência de RNA.

Figura $2.7 \boldsymbol{x}^{*}$ são os estados microscópicos obtidos ao invertermos todos os momentos de $\boldsymbol{x}$.

Figura 2.8 Distribuição para os valores do trabalho das trajetórias $\lambda_{F}$ e $\lambda_{R}$. O processo é reversível no ponto em que as curvas se interceptam, $W=\Delta F$.

Figura 3.1 Caminho da integração para as variáveis $p$ e $q$.

Figura 4.1 Evolução da entropia de uma partícula Browniana com as condições iniciais $x_{0}=v_{0}=0$. A taxa de variação da entropia se aproxima de valores negativos, ainda que fosse esperado um crescimento monotônico da entropia neste caso.

Figura 4.2 Comparação dos resultados numéricos (pontos) com as predições teóricas (linhas) para os valores esperados $\mu$ (a) e para as variâncias $\sigma$ (b) definidos na última seção. Para as integrações numéricas, foram escolhidos $k_{1}=k_{2}=2, \gamma=0,5 \mathrm{e}$ $m=\tau=T=\lambda=L_{0}=1$, com $x_{0}=v_{0}=0$, para os valores dos parâmetros. 
Figura 4.3 A evolução da entropia de uma partícula browniana, com condições iniciais fixadas, a diferentes temperaturas iniciais $T_{0}$. O gráfico inserido mostra a taxa de variação da entropia. Apesar da oscilação de $g(t)$ e $f(t)$, a entropia permanece sendo uma função monotônica (a). Em (b), apresentamos a evolução da entropia para diferentes valores da constante de amortecimento $\gamma$, enquanto que o gráfico inserido mostra a taxa de variação da entropia. Para valores suficientementes grandes de $\gamma$, o sistema apresenta fluxo reverso de informação, fazendo com que a entropia não seja mais uma função monotônica.

Figura 4.4 O espectro da entropia da Figura (4.3) (b) apresenta um pico acentuado em $\omega=0$, o que sugere o quase constante comportamento de $S(t)$.

Figura 4.5 Comportamento da derivada temporal da entropia. Os parâmetros utilizados são $\tau=0,0.01,0.1,0.2,0.5,1.0, \gamma=5 \mathrm{e}$ $m=1$. Observe que para $\tau=0$, temos $d S / d t>0$, portanto, não há fluxo reverso de informação..

Figura 4.6 Calor injetado (linha azul contínua) e calor dissipado (linha tracejada vermelha) durante o protocolo $L(t)$.

Figura 4.7 Calor total absorvido $\left(J_{i n j}(t)+J_{\text {diss }}(t)\right)$ pelo sistema durante o protocolo $L(t)$.

Figura 4.8 A distribuição de probabilidade para o calor injetado $J_{i n j} .73$

Figura 4.9 As flutuações do calor injetado obedecem ao respectivo teorema de flutuação.

Figura 4.10 Distribuição de probabilidade para o trabalho realizado por um sistema externo, de acordo com o protocolo $L(t)$.

Figura 4.11 Relação de flutuação obtida para o trabalho realizado por um sistema externo, de acordo com o protocolo $L(t)$.

Figura 4.12 Evolução da entropia instantânea $S(t)$ para o modelo não linear. A produção da entropia fica caracterizada pela inclinação da curva.

Figura 4.13 Análise espectral do comportamento de $S(t)$ para o modelo não linear.

Figura 4.14 O processo de expansão está ilustrado em (a), (b) e (c) e o processo de compressão, em (d), (e) e (f). Os últimos descrevem a etapa em que surgem os estados de "almoço grátis".

Figura C.1 Comportamento da parte real das raízes $\kappa$ (a) e parte imaginária das raízes $\kappa\left(\kappa_{I}\right)$ (b) como uma função de $\nu$ para diferentes valores de $\Gamma$ (na escala de $\tau$ ). Note que a parte real é sempre negativa e que $\kappa_{I}$ cresce para os valores de $\nu$ suficientemente grandes.

Figura C.2 Destacamos os valores dos parâmetros do sistema para os quais as oscilações podem ser observadas. Para um valor suficientemente grande de $\tau$, o discriminante sempre será negativo, daí o sistema apresentará oscilações. 
Figura C.3 Destacamos o comportamento da função de Green $g(t)$ e a função auxiliar $f(t)$ para os pontos A e B da Figura C.2. Quando o discriminante é positivo (linhas azuis), as funções sempre decaem. Quando os discriminante é negativo (linhas vermelhas), as funções apresentam um comportamento oscilatório. 


\section{Introdução}

Os Teoremas de Flutuação surgem na Mecânica Estatística como uma tentativa de responder à seguinte questão predominante [1]: como as equações de movimento microscópicas reversíveis podem conduzir a um comportamento macroscópico irreversível?

As considerações a respeito das relações de flutuações microscópicas com quantidades macroscópicas remontam o interesse da mecânica estatística de evidenciar a hipótese atomística da matéria. Os esforços de Einstein (1906) e Smoluchowski (1906) para a descrição teórica do movimento browniano [2, 3], somado aos resultados experimentais de Perrin [4], resultaram em uma evidência conclusiva da relação entre o coeficiente de difusão (uma quantidade macroscópica) com o número de Avogadro (relacionado à dinâmica microscópica) [5]. Além disso, ao descrever o movimento browniano, Einstein obteve a relação entre o coeficiente de difusão (propriedade de um sistema não perturbado) e a mobilidade de uma partícula browniana (medida da reação do sistema a uma pequena perturbação), o que constitui o primeiro exemplo de relação de flutuação-dissipação [5].

Em uma perspectiva mais moderna, as relações obtidas, como a relação para o trabalho devido a flutuações fora do equilíbrio, derivada por Bochkov \& Kuzovlev (1977) [6]; a probabilidade da violação da segunda lei da termodinâmica para sistemas fora do equilíbrio, proposta por Evans, Cohen \& Morriss (1993) [7]; a relação entre a energia livre e as flutuações do trabalho microscópico fora do equilíbrio, por Jarzinsky (1997) [8]; entre outras derivações, consolidaram a apreciação dos agora chamados Teoremas de Flutuação.

Os Teoremas de Flutuação são mais do que uma extrapolação microscópica da Termodinâmica macroscópica. Estas relações revelam novas características da segunda lei para pequenas escalas [9], relacionam flutuações fora do equilíbrio com quantidades no equilíbrio e quantificam a probabilidade de se violar a segunda lei da termodinâmica em sistemas de escala microscópica [1].

O interesse em aplicar os conceitos da Termodinâmica a sistemas microscópicos aumentou com a crescente intersecção de áreas de pesquisa da Biologia, Química, e Física. A saber, o avanço no conhecimento dos processos biomoleculares, em especial, os detalhes dos processos químico-mecânicos das proteínas 
[10].

Por outro lado, a relação da Teoria da Informação com os resultados teóricos, provenientes dos Teoremas de Flutuação, tem ficado cada vez mais estreita. Podemos considerar as ferramentas da Mecânica Estatística como uma forma de inferência estatística, à parte da teoria física associada a esta área de pesquisa; de modo que a física contribui para a descrição correta dos estados possíveis do sistema e suas características (modelo), e a Teoria da Informação toma o papel das inferências estatísticas decorrentes [11].

O modelo utilizado para descrever o sistema é um aspecto que não pode ser negligenciado. Apesar de que as leis da termodinâmica sejam independentes da abordagem, as equações que utilizamos para descrever o fenômeno físico encerram suposições específicas [9]. Neste sentido, um trabalho importante de J. Kurchan corrobora o fato de que os teoremas de flutuação também podem ser derivados pela abordagem das equações de Langevin [12].

Neste trabalho, vamos utilizar a equação de Langevin para descrever as flutuações estatísticas do nosso modelo. Diante disso, devemos especificar a tríade que compõe as suposições desta abordagem: sistema de interesse, sistema externo e banho térmico. O sumário a seguir, definido por Sekimoto, enfatiza os aspectos de cada componente desta tríade [13]:

- sistema de interesse (também chamado simplesmente de sistema neste trabalho): é o principal componente da tríade, do qual tomamos as informações dos seus estados representados por $x$ (posição) e $v$ (velocidade). A equação de Langevin descreve a evolução dos estados do sistema. A energia potencial e a energia cinética são consideradas parte dele;

- banho térmico: é um sistema em segundo plano, ao qual o sistema de interesse está conectado. O banho térmico é caracterizado por um único parâmetro, a temperatura $T$. Os princípios de conservação de energia, massa e momento não são aplicados a ele. O banho térmico retorna instantaneamente ao estado de equilíbrio, e não conserva memória da ação do sistema de interesse. A interação entre o sistema de interesse e o banho térmico é caracterizada pelo coeficiente de fricção e a temperatura. A força estocástica, ruído térmico, é proporcional a estes parâmetros;

- sistema externo: é um agente capaz de controlar macroscopicamente o sistema de interesse através de um parâmetro da posição contido na energia potencial. O termo "externo" significa que a evolução do parâmetro não é determinada pela equação de Langevin. O sistema externo não interfere na interação do sistema de interesse com o banho térmico. O sistema externo controla a troca de trabalho com o sistema de interesse. 
Com o intuito de facilitar a compreensão do texto, dividimos esta tese em três partes. No segundo capítulo, demonstramos uma forma de derivar um importante teorema de flutuação, a igualdade de Jarzinsky, e obtemos algumas importantes relações a partir desta última. No terceiro capítulo, a fim de coletar informações a partir das flutuações de estados fora do equilíbrio, introduzimos o modelo e as ferramentas estatísticas que nos serviram de base para o desenvolvimento do trabalho original desta tese. Por fim, no quarto capítulo apresentamos os resultados originais desta tese dentro deste contexto. 


\section{2}

\section{Igualdade de Jarzinsky}

O termo Teorema de Flutuação (TF) abrange uma gama de resultados que predizem teoricamente a produção de entropia [7, 14-16], flutuação do trabalho $[6,17,18]$, entre outras quantidades de sistemas conduzidos para fora do equilíbrio térmico [19]. Neste capítulo, vamos analisar um importante resultado conhecido como a Igualdade de Jarzinsky (IJ) [8]. Esta quantidade nos será útil para caracterizar os modelos propostos nos capítulos finais desta tese. A IJ é um tipo de TF para o trabalho realizado sobre o sistema. A IJ é, assim, um complemento interessante para os TFs relacionados à troca de calor e produção de entropia [14].

\section{1}

\section{Termodinâmica de sistemas microscópicos}

Devido à natureza estatística de larga escala, intrínseca à Termodinâmica, a primeira e a segunda lei da termodinâmica assumem um caráter proibitivo, no âmbito de simulações, para sistemas com muitos graus de liberdade. Por exemplo: um gás composto por $N \gg 10^{26}$ moléculas em equilíbrio térmico, confinado por paredes adiabáticas, sendo subitamente comprimido por um pistão móvel que, em seguida, é conduzido de volta à sua posição inicial, terá um acréscimo na sua energia interna, ou seja, $W>0$ ( $W$ é o trabalho realizado pelo movimento do pistão). A medida do trabalho $W$ é o valor esperado tomado sobre todas as partículas que compõem o gás. Portanto, é possível afirmar que certamente existem trajetórias no espaço de fase do gás para as quais $W<0$, isto é, a probabilidade dessas trajetórias não é nula. Contudo, quanto maior o número de partículas $N$, menor será a probabilidade de se observar trajetórias com esse comportamento.

Considerando agora um sistema com poucos graus de liberdade, realizando o mesmo procedimento em uma série de iterações hipotéticas (com o sistema em equilíbrio para cada nova iteração), é esperado que a média estatística do trabalho medido sobre todas as iterações satisfaça a inequação $\langle W\rangle>0$. Porém, é possível que sejam encontrados, em algumas raras observações, valores negativos para o trabalho realizado, ou seja, $W<0$.

Daí podemos concluir que as flutuações estatísticas, em torno da média 
das iterações, se tornam mais relevantes para sistemas com poucos graus de liberdade. Ainda, quando aplicamos as ferramentas da termodinâmica macroscópica para tais sistemas, com cada vez menos graus de liberdade, são reveladas novas características da primeira e da segunda da lei da termodinâmica [20].

\subsection{1}

\section{Termodinâmica macroscópica: energia livre de Helmholtz e trabalho}

Considerando um sistema puramente mecânico, o princípio da conservação da energia mecânica nos conduz à conclusão de que o trabalho realizado sobre o sistema é igual ao acréscimo na energia potencial do sistema. Já para um sistema termodinâmico, a troca de calor entre o sistema e a vizinhança torna a relação entre o trabalho realizado e variação de energia menos trivial.

A fim de expressarmos a relação trabalho/energia adequada, vamos supor um sistema de interesse em contato com um reservatório térmico à temperatura $T_{0}$, de modo que seja permitida a troca de calor $Q$ (o calor recebido pelo sistema e cedido pelo reservatório) entre o reservatório e o sistema. Ainda, o sistema e o reservatório só podem trocar calor entre si, e as paredes que confinam o sistema não são fixas. Logo, o sistema pode ser submetido a um trabalho volumétrico realizado por um sistema externo.

Após realizado trabalho sobre o sistema, é concedido um tempo suficientemente longo para que o sistema reequilibre termicamente com o reservatório. Isto implica que os pontos iniciais e finais (pontos de equilíbrio) do processo realizado sobre o sistema têm a mesma temperatura $T_{0}$. Porém, as temperaturas intermediárias não possuem necessariamente o mesmo valor.

O enunciado matemático da segunda lei da termodinâmica é expresso na forma $[21]$ :

$$
\Delta S+\Delta S_{0} \geq 0 .
$$

Na qual $\Delta S$ é a variação da entropia do sistema e $\Delta S_{0}$, do reservatório.

Utilizando o Teorema de Clausius [22], podemos concluir que $\Delta S_{0}=-\frac{Q}{T_{0}}$, posto que a temperatura $T_{0}$ do reservatório é constante. Daí a Eq. (2-1) pode ser reescrita tal como abaixo:

$$
Q-T_{0} \Delta S \leq 0
$$

A igualdade na inequação 2-2 acontece para processos de transferência de calor reversíveis. Portanto podemos escrever $Q^{r}=T_{0} \Delta S=Q+\delta q$. Na qual $Q^{r}$ é o calor para um processo reversível, Q é o calor para um processo irreversível e $\delta q$ é o calor dissipado [23]. A quantidade $\delta q$ é o trabalho dissipado transformado em calor. A definição de trabalho dissipado é descrita no fim desta seção. 
Agora podemos aplicar a formulação matemática da primeira lei da termodinâmica [21],

$$
\Delta U=Q+W
$$

ao sistema. O sinal positivo antes do termo do trabalho $W$ foi colocado propositalmente para salientar que o trabalho está sendo realizado sobre o sistema. Poderíamos também utilizar a forma usual da primeira lei da termodinâmica (com o sinal negativo) sem perda de generalização. Evidenciando o calor $Q$ na primeira lei e substituindo na Eq. (2-2), obtemos

$$
\Delta(U-T S)-W \leq 0
$$

Esta operação pode ser realizada porque a temperatura $T$ é a mesma no início e no fim do nosso processo. A quantidade $U-T S$ é a função matemática $F$ que representa a energia livre de Helmholtz. Então, podemos reescrever a inequação (2-4) na forma

$$
\Delta F \leq W
$$

A inequação (2-5) prediz que o trabalho realizado sobre o sistema não pode ser menor que a variação da energia livre de Helmholtz, entre o ponto inicial e o ponto final do processo. É chamado trabalho dissipado toda a quantidade que exceda a igualdade $W=\Delta F$ (processo reversível), ou seja [24],

$$
W_{\text {diss }}=W-\Delta F \text {. }
$$

É trivial que nem todo processo termodinâmico possua as características do exemplo dado anteriormente. Além disso, a inequação (2-5) não é a que contempla de forma mais genérica os processos descritos pela segunda lei da termodinâmica. Porém, neste trabalho focamos na classe de processos com características similares aos descritos nesta seção.

\subsection{2}

\section{Termodinâmica microscópica: energia livre de Helmholtz e trabalho}

Para descrevermos como obter informações de sistemas microscópicos, considerando as flutuações estatísticas que emergem desses sistemas, vamos utilizar um toy model para ilustrar o procedimento. Tanto o toy model utilizado, quanto as deduções decorrentes, são reproduções expandidas e inspiradas no review publicado por Jarzynski, e podem ser encontrado na íntegra em [20].

O sistema de interesse é formado por três partículas (representados pelos círculos em branco). As coordenadas $z_{i}(i=1,2$ e 3$)$ são as distâncias de cada partícula de uma parede fixa. Uma coodernada adicional é o parâmetro do trabalho realizado sobre o sistema, identificado por $\lambda$. O parâmetro $\lambda$ é 


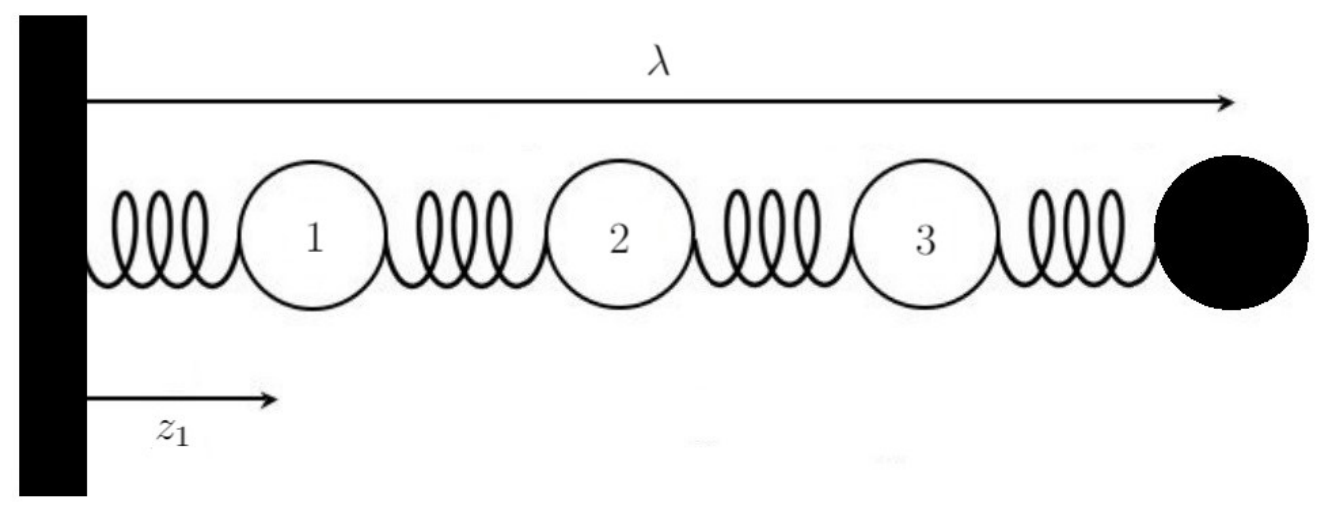

Figura 2.1: Os círculos enumerados representam as três partículas do sistema. As coordenada $z_{1}, z_{2}$ e $z_{3}$ são as distâncias de cada partícula até a parede fixa (retângulo na vertical), explicitada para $z_{1}$. O círculo preto é o parâmetro do trabalho. A posição $\lambda$ é manipulada externamente seguindo um protocolo. As molas representam as interações par-a-par entre as partículas. Os graus de liberdade do reservatório térmico não estão ilustrados.

controlado por um agente externo e descreve a posição, orientação ou força externa de um corpo que interage com o sistema. Na figura, o parâmetro do trabalho é representado pelo círculo preto a uma distância $\lambda$ da parede fixa. O parâmetro $\boldsymbol{x}$ é um estado microscópico do sistema, a saber, as posições e os momentos de cada partícula do sistema, representados por um ponto no espaço de fase. $\boldsymbol{y}$ é um estado microscópico de reservatório térmico. Sendo assim, a Hamiltoniana para o sistema e reservatório térmico combinados, considerando as variáveis clássicas dos graus de liberdade assumidas, toma a seguinte forma:

$$
\boldsymbol{H}(\boldsymbol{x}, \boldsymbol{y} ; \lambda)=\mathcal{H}(\boldsymbol{x} ; \lambda)+\mathcal{H}_{\text {res }}(\boldsymbol{y})+h_{\text {int }}(\boldsymbol{x}, \boldsymbol{y}),
$$

na qual $\mathcal{H}(\boldsymbol{x} ; \lambda)$ é a Hamiltoniana do sistema de interesse (incluindo a interação com o agente externo mediado pelo parâmetro do trabalho $\lambda), \mathcal{H}_{\text {res }}(\boldsymbol{y})$ é a Hamiltoniana do reservatório e $h_{\text {int }}(\boldsymbol{x}, \boldsymbol{y})$ é a Hamiltoniana de interação entre o sistema e o reservatório térmico. Para este modelo, $\boldsymbol{x}=\left(z_{1}, z_{2}, z_{3}, p_{1}, p_{2}, p_{3}\right)$ e

$$
\mathcal{H}(\boldsymbol{x} ; \lambda)=\sum_{k=0}^{3} u\left(z_{k+1}-z_{k}\right)+\sum_{i=1}^{3} \frac{p_{i}^{2}}{2 m},
$$

na qual $u(\bullet)$ é o potencial de interação par a par da associação em série, $z_{0} \equiv 0$ e $z_{4} \equiv \lambda$.

Supondo um sistema microscópico análago ao descrito anteriormente, vamos assumir que inicialmente o sistema e o reservatório estão em equilíbrio térmico à temperatura $T$, e o parâmetro do trabalho está posicionado no estado $\lambda=A$. O sistema e o reservatório estão inicialmente em um estado 
microscópico $\left(\boldsymbol{x}_{\mathbf{0}}, \boldsymbol{y}_{\mathbf{0}}\right)$, distribuídos pela forma canônica do espaço de fase [25]:

$$
p_{A}\left(\boldsymbol{x}_{\mathbf{0}}, \boldsymbol{y}_{\mathbf{0}}\right)=\frac{1}{Y_{A}} \exp \left[-\beta \boldsymbol{H}\left(\boldsymbol{x}_{\mathbf{0}}, \boldsymbol{y}_{\mathbf{0}} ; \lambda=A\right)\right] .
$$

$\mathrm{O}$ fator de normalização $Y_{A}$ provém da identidade

$$
Y_{\lambda}=\int d \boldsymbol{x} d \boldsymbol{y} \exp [-\beta \boldsymbol{H}(\boldsymbol{x}, \boldsymbol{y} ; \lambda)]
$$

na qual a integral é realizada sobre todo o espaço de fase e $\beta=1 / k_{B} T$ [26]. Quando o parâmetro do trabalho está fixado em $\lambda, Y_{\lambda}$ é a função de partição para o estado de equilíbrio do sistema e do reservatório combinados [27].

A energia livre de Helmholtz associada ao sistema é dada pela equação usual $F=-\beta^{-1} \ln Z_{\lambda}[28]$. O termo

$$
Z_{\lambda}=\int d \boldsymbol{x} \exp [-\beta \mathcal{H}(\boldsymbol{x} ; \lambda)]
$$

é a função de partição da Hamiltoniana do sistema de interesse quando o parâmetro do trabalho está posicionado em $\lambda$. Daí podemos obter a variação da energia como segue:

$$
\Delta F \equiv F_{B}-F_{A}=-\beta^{-1} \ln \frac{Z_{B}}{Z_{A}} .
$$

Partindo do estado de equilíbrio $\left(\boldsymbol{x}_{\mathbf{0}}, \boldsymbol{y}_{\mathbf{0}} ; A\right)$, o agente externo manipula o parâmetro do trabalho de acordo com o protocolo $\lambda(t)$. Enquanto o sistema e o reservatório evoluem microscopicamente no tempo, o parâmetro varia de $\lambda=A$, em $t=0$, até $\lambda=B$, em $t=\tau$. Durante este processo, o sistema e o reservatório realizam uma trajetória $\Gamma_{t}$ pelo espaço de fase total (sistema e reservatório combinados). O protocolo $\lambda(t)$ define como o agente externo atua sobre o sistema durante o processo. Já $\Gamma_{t}$, define como o sistema e o reservatório respondem, no âmbito dos estados microscópicos, ao estímulo.

Para uma trajetória $\Gamma_{t}$ sobre o espaço de fase, temos a seguinte variação da Hamiltoniana do sistema e reservatório:

$$
\begin{aligned}
\boldsymbol{H}(\boldsymbol{x}(\tau), \boldsymbol{y}(\tau) ; B)-\boldsymbol{H}\left(\boldsymbol{x}_{\mathbf{0}}, \boldsymbol{y}_{\mathbf{0}} ; A\right) & =\int_{0}^{\tau} d t \frac{d}{d t} \boldsymbol{H}(\boldsymbol{x}(t), \boldsymbol{y}(t) ; \lambda(t)) \\
& =\int_{0}^{\tau} d t \dot{\lambda} \frac{\partial}{\partial \lambda} \boldsymbol{H}(\boldsymbol{x}(t), \boldsymbol{y}(t) ; \lambda(t)) \\
& =\int_{0}^{\tau} d t \dot{\lambda} \frac{\partial}{\partial \lambda} \mathcal{H}(\boldsymbol{x}(t) ; \lambda(t)) .
\end{aligned}
$$

Na qual $\boldsymbol{x}(t)$ e $\boldsymbol{y}(t)$ são os pontos no espaço de fase no tempo $t$ para o sistema e o reservatório, respectivamente, contidos na trajetória $\Gamma_{t}$. A passagem da primeira para a segunda linha da Eq. 2-13 se deve a uma propriedade conhecida das equações de movimento de Hamilton, isto é, $\frac{d \boldsymbol{H}}{d t}=\frac{\partial \boldsymbol{H}}{\partial t}[29]$. A terceira linha provém da dependência das Hamiltonianas no lado direito da Eq. 4-13. 
Ao realizarmos o processo de $\lambda=A$, em $t=0$, até $\lambda=B$, em $t=\tau$, pela trajetória $\Gamma_{t}$, a variação da energia interna do sistema é dada pela seguinte quantidade:

$$
\begin{aligned}
d \mathcal{H}(\boldsymbol{x}(t) ; \lambda(t)) & =\dot{\boldsymbol{x}} \frac{\partial}{\partial \boldsymbol{x}} \mathcal{H}(\boldsymbol{x}(t) ; \lambda(t)) d t+\dot{\lambda} \frac{\partial}{\partial \lambda} \mathcal{H}(\boldsymbol{x}(t) ; \lambda(t)) d t(2-14) \\
\mathcal{H}(\boldsymbol{x}(\tau) ; B)-\mathcal{H}\left(\boldsymbol{x}_{\mathbf{0}} ; A\right) & =\int_{0}^{\tau} d t \dot{\boldsymbol{x}} \frac{\partial}{\partial \boldsymbol{x}} \mathcal{H}(\boldsymbol{x}(t) ; \lambda(t))+\int_{0}^{\tau} d t \dot{\lambda} \frac{\partial}{\partial \lambda} \mathcal{H}(\boldsymbol{x}(t) ; \lambda(t))
\end{aligned}
$$

Podemos interpretar o segundo termo do lado direito da Eq. 2-15 como o trabalho realizado pelo agente externo sobre o sistema [18]. Já o primeiro termo, podemos interpretar como o calor cedido ou absorvido do reservatório térmico pelo sistema. Pois, se realizássemos este procedimento em um modelo similar, desta vez sem o contato do sistema com o reservatório térmico, teríamos o mesmo termo para o trabalho sobre o sistema dado pela Eq. 2-15, e seu valor seria igual à variação da energia interna do sistema. Além disso, este termo corresponde à atuação de uma imensa quantidade de graus de liberdade entre o sistema e o reservatório, ou seja, uma forma desordenada de troca de energia: o calor. Portanto, o primeiro termo do lado direito da Eq. 2-15 é a soma de toda a variação da energia interna do sistema devido ao contato com o reservatório térmico [30, 31].

A descrição microscópica do trabalho realizado sobre o sistema e do calor absorvido ou cedido pelo sistema é definida somente em termos da trajetória de $\boldsymbol{x}(t)$ no espaço de fase. Portanto, a Eq. 2-15 pode ser vista como a primeira lei da termodinâmca (Eq. 2-3) para este sistema, na qual:

$$
\begin{aligned}
W & \equiv \int_{0}^{\tau} d t \dot{\lambda} \frac{\partial}{\partial \lambda} \mathcal{H}(\boldsymbol{x}(t) ; \lambda(t)) \\
Q & \equiv \int_{0}^{\tau} d t \dot{\boldsymbol{x}} \frac{\partial}{\partial \boldsymbol{x}} \mathcal{H}(\boldsymbol{x}(t) ; \lambda(t)) .
\end{aligned}
$$

Daí podemos concluir que para sabermos a quantidade de calor, absorvido ou cedido pelo sistema de interesse, e o trabalho realizado sobre ele, basta nos importarmos somente com os graus de liberdade do próprio sistema.

Ainda podemos extrair mais uma informação da Eq. 2-13 e Eq. 2-16, que segue:

$$
W=\boldsymbol{H}\left(\boldsymbol{x}_{\tau}, \boldsymbol{y}_{\tau} ; B\right)-\boldsymbol{H}\left(\boldsymbol{x}_{\mathbf{0}}, \boldsymbol{y}_{\mathbf{0}} ; A\right),
$$

isto é, a quantidade de trabalho realizado sobre o sistema, em termos da sua evolução microscópica, é igual à variação da Hamiltoniana do sistema e reservatório térmico combinados (condicionada à evolução da Hamiltoniana do 
sistema e reservatório combinados no mesmo período do trabalho realizado). As equivalências $\boldsymbol{x}_{\tau} \equiv \boldsymbol{x}(\tau)$ e $\boldsymbol{y}_{\tau} \equiv \boldsymbol{y}(\tau)$ serão utilizadas daqui em diante por conveniência meramente estética.

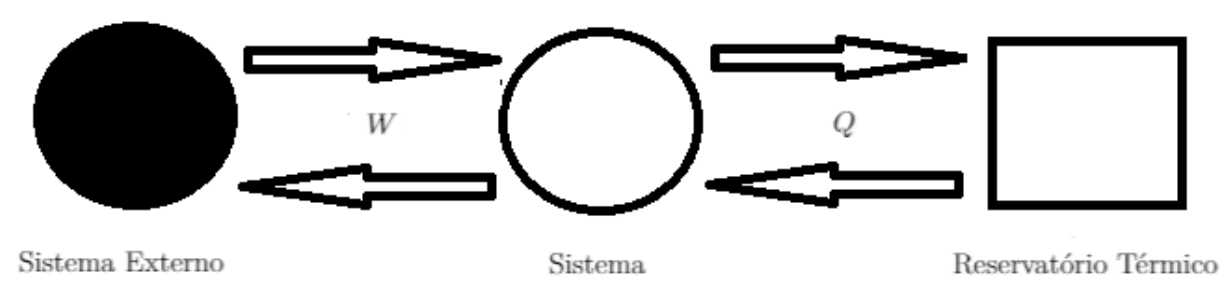

Figura 2.2: Ilustração das trocas de energia entre o sistema de interesse e o sistema externo, e entre o sistema de interesse e o reservatório térmico.

Pode-se argumentar que as últimas conclusões são válidas somente quando a Hamiltonana de interação entre o reservatório e o sistema, $\mathcal{H}_{\text {int }}(\boldsymbol{x}, \boldsymbol{y})$, é suficientemente fraca para ser negligenciada. De fato, a distribuição da Eq. 2-11 será modificada de acordo com a proporção [32]:

$$
p_{\lambda}^{\dagger}(\boldsymbol{x}) \propto \exp \left[-\beta \mathcal{H}^{\dagger}(\boldsymbol{x} ; \lambda)\right]
$$

na qual

$$
\mathcal{H}^{\dagger}(\boldsymbol{x} ; \lambda)=\mathcal{H}(\boldsymbol{x} ; \lambda)+\Psi((\boldsymbol{x} ; T) .
$$

A função $\Psi((\boldsymbol{x} ; T)$ é o custo energético requerido para inserir o sistema em um reservatório térmico. Por exemplo: a energia associada ao rearranjamento da água após inserir uma biomolécula em um meio aquoso [33]. Neste caso, o sistema e o reservatório térmico compartilham a energia do termo da Hamiltoniana de interação, e não é evidente se esta energia pertence ao sistema ou ao reservatório térmico. Apesar disso, essa troca na Hamiltoniana do sistema somente mudaria a definição do calor na Eq. (2-17), mas a definição do trabalho na Eq. (2-16) permaneceria a mesma, pois $\frac{\partial \mathcal{H}^{\dagger}}{\partial \lambda}=\frac{\partial \mathcal{H}}{\partial \lambda}$. Portanto, para as derivações posteriores, essa troca não tem efeitos.

O processo termodinâmico relatado anteriormente foi discutido no âmbito de uma única realização. Agora vamos estender esse procedimento para muitas realizações, até mesmo infinitas, de forma que possamos obter informações estatísticas destas iterações. Para cada repetição, o sistema e o reservatório térmico estão em equilíbrio e o mesmo processo é realizado, variando o 
parâmetro do trabalho de $\lambda=A$, em $t=0$, até $\lambda=B$, em $t=\tau$, seguindo o mesmo protocolo $\lambda(t)$. No entanto, a trajetória do sistema e do reservatório térmico pelo espaço de fase, $\Gamma_{t}$, será possivelmente diferente para cada nova iteração, uma vez que os estados microscópicos iniciais serão diferentes para cada realização devido à distribuição da Eq. (2-9).

Ao considerarmos as infinitas repetições, obtemos um ensemble estatístico das realizações do processo constituído por diferentes trajetórias $\Gamma_{t}^{1}, \Gamma_{t}^{2}$, $\Gamma_{t}^{3} \ldots$ E para cada uma destas trajetórias, calculamos o trabalho $W^{1}, W^{2}, W^{3} \ldots$ Assim, o valor esperado do trabalho realizado tomado sobre esse ensemble é dado por:

$$
\langle W\rangle \equiv \int d W \rho(W) W
$$

na qual $\rho(W)$ é a distribuição de probabilidade do trabalho realizado sobre o sistema. A quantidade $\langle W\rangle$ é igual a $W$ na inequação (2-5).

\section{2}

\section{Informação associada a flutuações fora do equilíbrio}

Com o conjunto dos valores $W^{n}$ para o trabalho definidos na última seção, podemos construir o valor médio da quantidade $e^{-\beta W}$. Esta quantidade é dada explicitamete por:

$$
\left\langle e^{-\beta W}\right\rangle=\lim _{N \rightarrow \infty} \frac{1}{N} \sum_{n=1}^{N} e^{-\beta W^{n}} .
$$

Na qual a temperatura $T$ em $\beta=1 / k_{B} T$ é a temperatura inicial do sistema e do reservatório térmico.

Para calcularmos a quantidade da Eq. (2-22), deixamos que o sistema evolua de $t=0$ até $t=\tau$ condicionado à Hamiltoniana $\mathcal{H}(\boldsymbol{x}(t) ; \lambda(t))$, com o sistema em $t=0$ em equilíbrio térmico à temperatura $T$. Neste período, o parâmetro do trabalho é deslocado de $\lambda(0)=A$ até $\lambda(\tau)=B$, conforme o protocolo $\lambda(t)$. Introduzimos a função $\tilde{W}\left(\boldsymbol{x}_{\mathbf{0}}, \boldsymbol{y}_{\mathbf{0}}\right)$, que representa o trabalho realizado sobre o sistema sobre uma trajetória $\Gamma_{t}$, partindo das condições iniciais $\left(\boldsymbol{x}_{\mathbf{0}}, \boldsymbol{y}_{\mathbf{0}}\right)$. São as condições iniciais que determinam exclusivamente a trajetória $\Gamma_{t}$ no espaço de fase. A cada trajetória o valor do trabalho pode ser calculado pela Eq. (2-16) ou pela Eq. (2-18). Daí podemos calcular o valor de Eq.(2-22) da seguinte forma: 


$$
\begin{aligned}
\left\langle e^{-\beta W}\right\rangle & =\int d \boldsymbol{x}_{\mathbf{0}} d \boldsymbol{y}_{\mathbf{0}} p\left(\boldsymbol{x}_{\mathbf{0}}, \boldsymbol{y}_{\mathbf{0}}\right) \exp \left[-\beta \tilde{W}\left(\boldsymbol{x}_{\mathbf{0}}, \boldsymbol{y}_{\mathbf{0}}\right)\right] \\
& \left.=\int d \boldsymbol{x}_{\mathbf{0}} d \boldsymbol{y}_{\mathbf{0}} \frac{1}{Z_{A}} \exp \left[-\beta\left\{\boldsymbol{H}\left(\boldsymbol{x}_{\mathbf{0}}, \boldsymbol{y}_{\mathbf{0}} ; \lambda=A\right)\right]+\beta \tilde{W}\left(\boldsymbol{x}_{\mathbf{0}}, \boldsymbol{y}_{\mathbf{0}}\right)\right\}\right] \\
& =\int d \boldsymbol{x}_{\mathbf{0}} d \boldsymbol{y}_{\mathbf{0}} \frac{1}{Z_{A}} \exp \left[-\beta \boldsymbol{H}^{\left(\boldsymbol{x}_{\mathbf{0}}, \boldsymbol{y}_{\mathbf{0}}\right)}\left(\boldsymbol{x}_{\boldsymbol{\tau}}, \boldsymbol{y}_{\boldsymbol{\tau}} ; \lambda=B\right)\right]
\end{aligned}
$$

Para a passagem da primeira para a segunda linha na Eq. (2-23), foi utilizada a definição de distribuição da Eq. (2-9). Já para a passagem da segunda para a terceira linha, o importante resultado obtido na Eq. (2-18) para o trabalho foi aplicado. A Hamiltonina $\boldsymbol{H}^{\left(x_{0}, y_{0}\right)}(\bullet)$ é determinística e o índice sobrescrito representa que o estado microscópico final no espaço de fase $\left(\boldsymbol{x}_{\boldsymbol{\tau}}, \boldsymbol{y}_{\tau}\right)$ foi alcançado partindo das condições iniciais dos estados microscópicos em $\left(\boldsymbol{x}_{\mathbf{0}}, \boldsymbol{y}_{\mathbf{0}}\right)$.

A fim de calcularmos a integral da Eq. (2-23), vamos fazer a seguinte mudança de variável:

$$
d \boldsymbol{x}_{\mathbf{0}} d \boldsymbol{y}_{\mathbf{0}}=\left|\frac{\partial\left(\boldsymbol{x}_{\mathbf{0}}, \boldsymbol{y}_{\mathbf{0}}\right)}{\partial\left(\boldsymbol{x}_{\tau}, \boldsymbol{y}_{\tau}\right)}\right| d \boldsymbol{x}_{\tau} d \boldsymbol{y}_{\tau}
$$

na qual $\left|\frac{\partial\left(\boldsymbol{x}_{0}, \boldsymbol{y}_{\mathbf{0}}\right)}{\partial\left(\boldsymbol{x}_{\tau}, \boldsymbol{y}_{\tau}\right)}\right|$ é o Jacobiano da transformação [34].

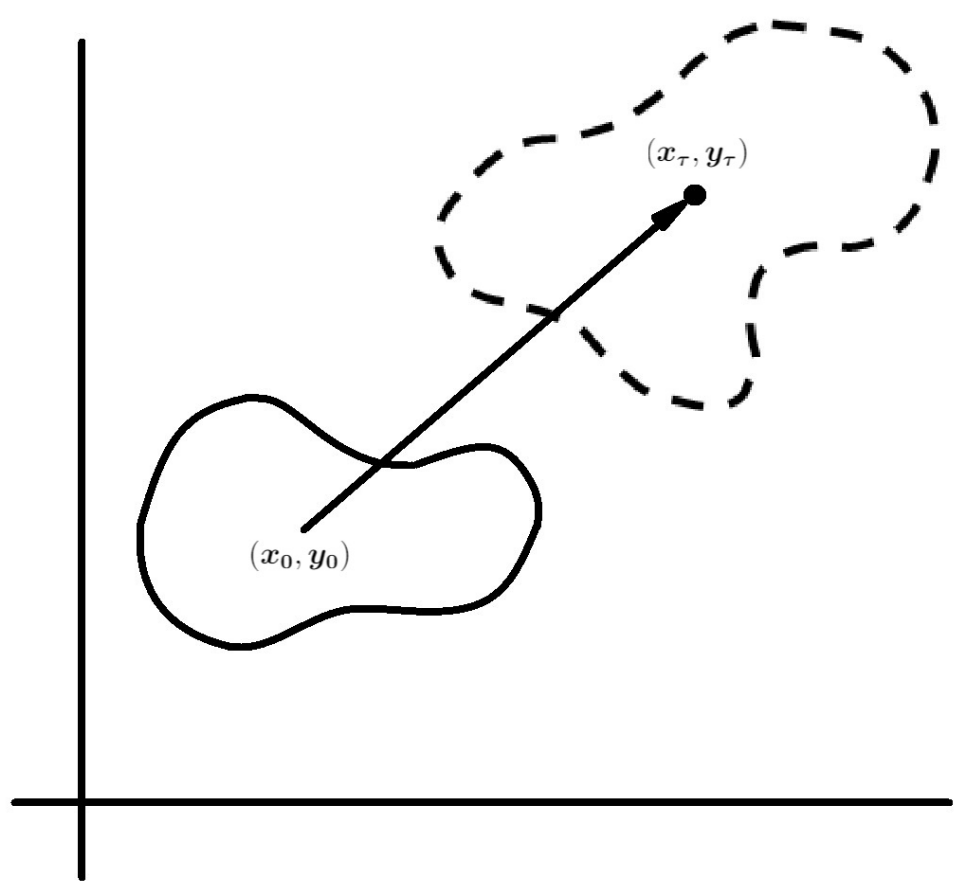

Figura 2.3: Movimento do volume no espaço de fase. A densidade dos sistemas do ensemble na vizinhança de um ponto do espaço de fase não muda com o tempo. 
Sabe-se na literatura que um volume infinitesimal do espaço de fase é um invariante frente uma transformação canônica. Portanto, esta quantidade não se altera ao longo da evolução temporal do sistema [35]. Esta conclusão é resultado do conhecido Teorema de Liouville [36]:

$$
\frac{\partial \rho}{\partial t}+\sum_{i=1}^{n}\left[\frac{\partial H}{\partial p_{i}} \frac{\partial \rho}{\partial q_{i}}-\frac{\partial H}{\partial q_{i}} \frac{\partial \rho}{\partial p_{i}}\right]=0,
$$

na qual $\rho$ é a distribuição de probabilidade para o espaço de fase e $H$ a Hamiltoniana do sistema de interesse. Então, o Jacobiano da Eq (2-24) deve ser igual a 1 .

Aplicando as últimas conclusões à Eq. (2-23) podemos escrever

$$
\begin{aligned}
\left\langle e^{-\beta W}\right\rangle & =\int d \boldsymbol{x}_{\tau} d \boldsymbol{y}_{\tau} \frac{1}{Y_{A}} \exp \left[-\beta \boldsymbol{H}^{\left(x_{0}, y_{0}\right)}\left(\boldsymbol{x}_{\tau}, \boldsymbol{y}_{\tau} ; \lambda=B\right)\right] \\
& =\frac{Y_{B}}{Y_{A}}
\end{aligned}
$$

na qual Eq. (A-3) foi utilizada para definirmos $Y_{A}$ e $Y_{B}$.

O último resultado já nos evidencia uma importante conclusão: embora a distribuição dos valores do trabalho $\rho(W)$ dependa geralmente do protocolo $\lambda(t)$, a quantidade $\left\langle e^{-\beta W}\right\rangle$ só depende dos parâmetros $\lambda=A, \lambda=B$ e $T$, ou seja, parâmetro inicial e final do trabalho, e a temperatura inicial do sistema, respectivamente.

As funções de partição $Y_{A}$ e $Y_{B}$ dependem da Hamiltoniana do sistema e do reservatório térmico combinados. Vamos agora substituí-las por uma expressão que dependa somente da Hamiltoniana do sistema. Apesar dessa troca ser válida somente quando a Hamiltoniana de interação $\mathcal{H}_{\text {int }}(\boldsymbol{x}, \boldsymbol{y})$ pode ser negligenciada, o resultado obtido não perde a sua generalidade. A demonstração desta afirmação, e mais detalhes sobre o potencial de interação, pode ser conferida no apêndice A.1. Portanto, podemos escrever a Eq. (2-26) na forma:

$$
\frac{Y_{B}}{Y_{A}}=\frac{\int d \boldsymbol{x} d \boldsymbol{y} \exp [-\beta \boldsymbol{H}(\boldsymbol{x}, \boldsymbol{y} ; A)]}{\int d \boldsymbol{x} d \boldsymbol{y} \exp [-\beta \boldsymbol{H}(\boldsymbol{x}, \boldsymbol{y} ; B)]} \approx \frac{\int d \boldsymbol{x} \exp [-\beta \mathcal{H}(\boldsymbol{x} ; A)]}{\int d \boldsymbol{x} \exp [-\beta \mathcal{H}(\boldsymbol{x} ; B)]}=\frac{Z_{B}}{Z_{A}}=e^{-\beta \Delta F} .
$$

O resultado do lado extremo direito da equação se deve à substituição possibilitada pela definição da energia livre de Helmholtz da Eq. (2-12). O termo referente à Hamiltoniana do reservatório $\mathcal{H}_{\text {res }}(\boldsymbol{y})$ é eliminado da razão $Z_{B} / Z_{A}$ porque não depende de $\lambda$.

Associando o termo do lado esquerdo da Eq. (2-23) e o resultado do lado extremo direito da Eq. (2-27), obtemos a seguinte igualdade: 


$$
\left\langle e^{-\beta W}\right\rangle=e^{-\beta \Delta F}
$$

Esta relação é um teorema de flutuação para o trabalho conhecido como a Igualdade de Jarzynski. Desde a sua dedução original [8], este resultado tem sido derivado de diversas maneiras, utilizando uma variedade de equações do movimento para modelar a dinâmica microscópica [37-41]. Também tem sido comprovada a sua validade experimentalmente [42-45].

A IJ descreve que se repetirmos um processo muitas vezes, podemos determinar a variação da energia livre pelas medidas obtidas de um sistema conduzido para fora do equilíbrio. O sinal de igualdade na inequação (2-5) é atribuído somente para processos reversíveis. A IJ nos possibilita também derivar uma igualdade para processos irreversíveis, a saber:

$$
-k_{B} T \ln \left\langle e^{-W / k_{B} T}\right\rangle=\Delta F .
$$

Entretanto, o valor de $\Delta F$ não pode ser encontrado com uma única medida, mas, sim, pelas flutuações estatísticas do trabalho. A ideia central da IJ, e de outros teoremas de flutuação, é que flutuações fora do equilíbrio contêm informações de estados de equilíbrio. Combinando a IJ com a desigualdade de Jensen, $\langle\exp x\rangle \geq \exp \langle x\rangle$, temos novamente a relação entre trabalho e energia livre de Helmholtz da Eq. (2-5).

Podemos agora obter um resultado muito importante utilizando a IJ [46]: a probabilidade de que a medida do valor do trabalho seja menor que a quantidade $\Delta F-\chi$, na qual $\chi$ é um valor positivo arbitrário com unidade de energia. A probabilidade a ser calculada é a área da cauda da distribuição de probabilidade $\rho(W)$ na Eq.(2-21). A Figura (2.2) ilustra esta distribuição.

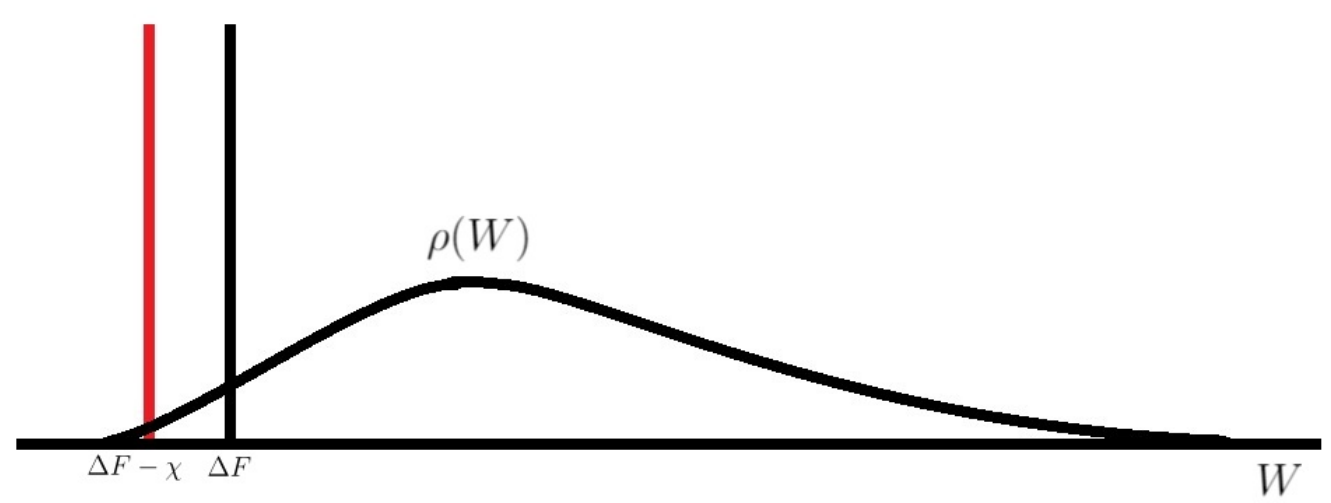

Figura 2.4: Distribuição do trabalho $\rho(W)$ para um sistema microscópico sujeito a um processo irreversível. A região na qual $W<\Delta F$ é a chamada proibitiva 
Para calcular a probabilidade de observar os pontos à esquerda de $W=\Delta F$, fazemos

$$
\begin{aligned}
P(W<\Delta F-\chi) & =\int_{-\infty}^{\Delta F-\chi} \rho(W) d W \\
& \leq \int_{-\infty}^{\Delta F-\chi} \rho(W) e^{\beta(\Delta F-\chi-W)} d W \\
& \leq e^{\beta(\Delta F-\chi)} \int_{-\infty}^{\infty} \rho(W) e^{-\beta W} d W \\
& \leq e^{\beta(\Delta F-\chi)} e^{\beta(\Delta F)} \\
& \leq e^{-\beta \chi} .
\end{aligned}
$$

A IJ foi utilizada na passagem da terceira para a quarta linha da Eq. (2-30). A cauda do lado esquerdo da distribuição $\rho(W)$ decai exponencialmente. Assim, os valores microscópicos para o trabalho $W<\Delta F$ podem ser observados.

Poderíamos afirmar aqui que haveria uma "violação" da segunda lei da termodinâmica, pois há probabilidade não nula de observar valores do trabalho para a região proibitiva. Porém, a validade da segunda lei é somente para medidas macroscópicas. Ainda assim, os valores do trabalho que estão a poucas unidades de $\beta^{-1}$ abaixo de $\Delta F$ são raras de observar, tornando a região $\chi \gg \beta$ efetivamente proibitiva, e indo ao encontro das experiências macroscópicas. A segunda lei é satisfeita sobre o valor esperado calculado e não é violada em uma escala macroscópica.

\section{3}

\section{Evolução estocástica e simetria microscópica}

Considerando os resultados analisados na última seção, vamos propor dois processos distintos para o mesmo sistema microscópico já descrito: o primeiro é o mesmo detalhado na última seção, no qual variamos o parâmetro do trabalho de $\lambda_{F}=A$, em $t=0$, até $\lambda_{F}=B$, em $t=\tau$, usando o protocolo $\lambda_{F}(t)$. Depois de ser concedido tempo suficiente para o sistema estar em equilíbrio térmico em $\lambda_{F}=B$, o segundo processo consiste em variar o parâmetro do trabalho de $\lambda_{R}=B$, em $t=0$, até $\lambda_{R}=A$, em $t=\tau$, ou seja, o processo reverso. Daí o subíndice em $\lambda_{F}$ e $\lambda_{R}$, do inglês "forward" e "reverse", respectivamente [18]. Contudo, desta vez, durante o intervalo $0 \leq t \leq \tau$, o sistema é termicamente isolado do reservatório. Tanto em $\lambda=A$ quanto em $\lambda=B$, o sistema é preparado em um estado de equilíbrio à temperatura inicial $T$ antes de se realizar cada processo. Durante o processo $\lambda=B$ até $\lambda=A$, é utilizado o protocolo de "tempo-reverso" definido por: 


$$
\lambda_{R}(t)=\lambda_{F}(\tau-t)
$$

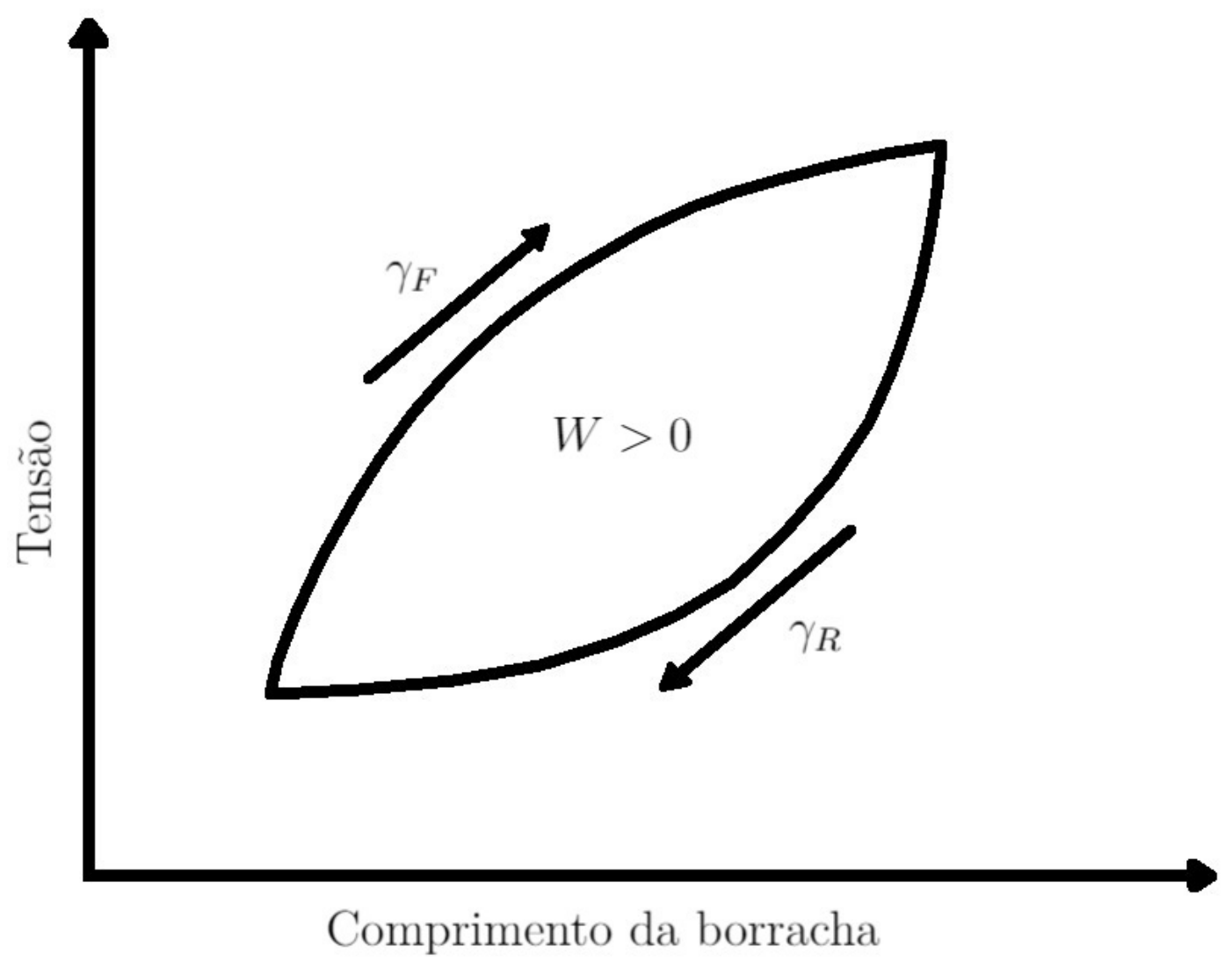

Figura 2.5: Uma analogia para a produção de trabalho: esticar e contrair um elástico de borracha. Se esticássemos a borracha, a temperatura e a tensão seriam maiores do que no processo reverso, a contração. É um exemplo de ciclo de histerese no qual $W>0$.

Os dois processos são realizados em sequência, sendo que o processo reverso é realizado por último, concedendo tempo suficiente para que o sistema entre em equilíbrio com o reservatório térmico no fim de cada processo. Temos neste caso o chamado ciclo de histerese: em um processo irreversível, o sistema evolui em uma sequência de estados durante o processo de protocolo $\lambda_{F}$, mas o processo reverso (protocolo $\lambda_{R}$ ) percorre uma trajetória de volta diferente. Portanto, o trabalho do ciclo completo será maior que zero, como ilustrado na Figura 2.5. Então, a inequação (2-5) para os dois processos combinados resulta no intervalo

$$
-\langle W\rangle_{R} \leq \Delta F \leq\langle W\rangle_{F}
$$

na qual $\langle W\rangle$ é o valor esperado do trabalho calculado sobre a distribuição dos valores do trabalho para cada processo. Assim, concluímos que para o ciclo 
completo:

$$
\langle W\rangle_{R}+\langle W\rangle_{F} \geq 0
$$

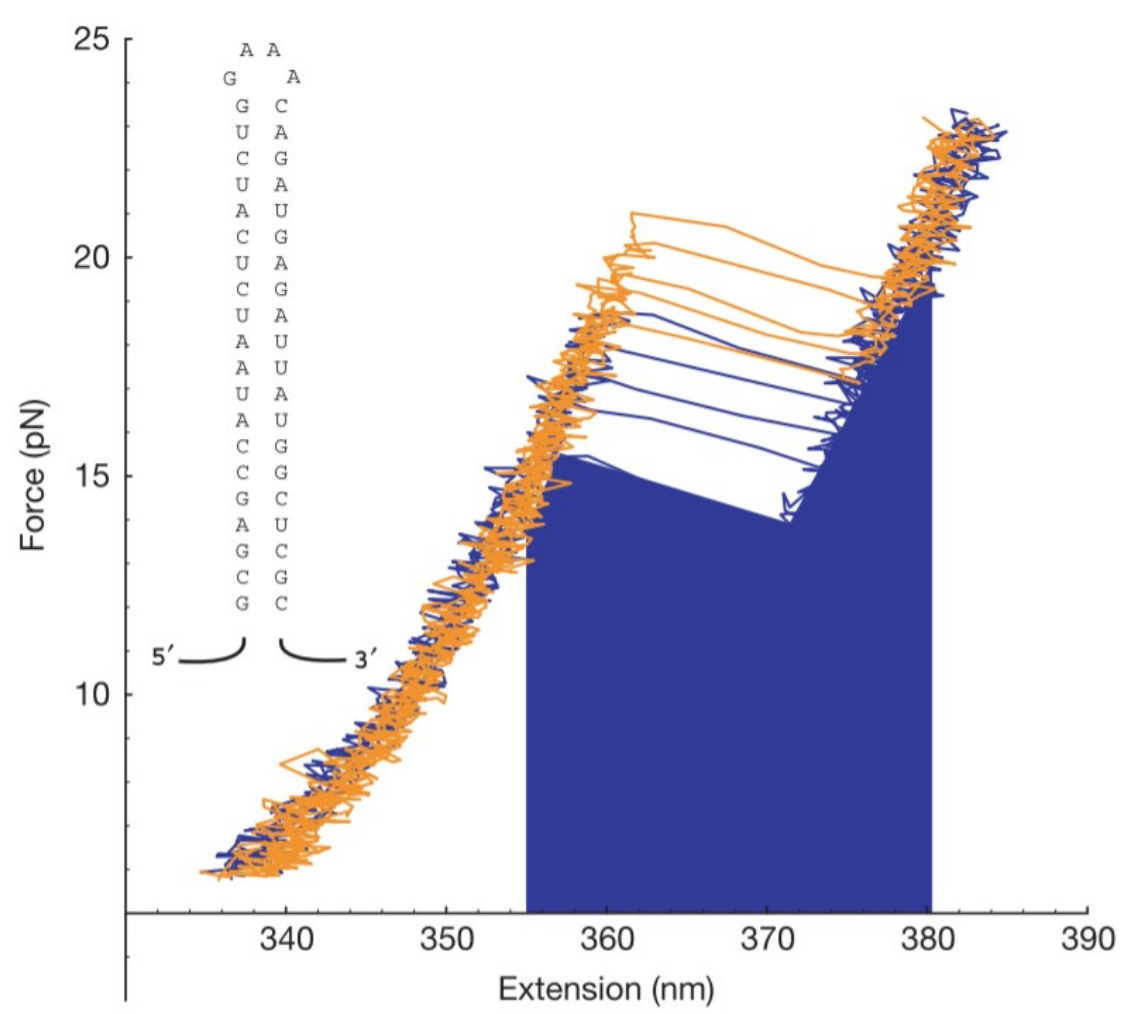

Figura 2.6: Trajetória da contração (azul) e expansão (laranja) de uma sequência de RNA.

Fonte: Collin, Delphine, et al. "Verification of the Crooks fluctuation theorem and recovery of RNA folding free energies." Nature 437.7056 (2005): 231-234.

Vamos considerar agora as trajetórias de cada processo, denotando por $\gamma_{R} \equiv\left\{\boldsymbol{x}_{R}(t) ; 0 \leq t \leq \tau\right\}$ a trajetória no sentido reverso. Assim, $\gamma_{F}$ é seu conjugado no qual

$$
\boldsymbol{x}_{R}(t)=\boldsymbol{x}_{F}^{*}(t-\tau) .
$$

A Figura (2.7) ilustra os pares conjugados das trajetórias $\gamma_{F}$ e $\gamma_{R}$. Uma analogia para esclarecer as trajetórias é: se pudessemos filmar a trajetória $\gamma_{F}$, $\gamma_{R}$ seria a trajetória que veríamos se víssemos o filme de trás para frente.

A probabilidade de realizarmos determinada trajetória será diferente para cada processo. Vamos definir como $P_{F}\left[\gamma_{F}\right]$ a probabilidade de observarmos a trajetória $\gamma_{F}$. Do mesmo modo, teremos a probabilidade $P_{R}\left[\gamma_{R}\right]$ para a trajetória reversa $\gamma_{R}$. O valor do trabalho realizado em cada trajetória será $W\left[\gamma_{F}\right] \equiv W_{F}$ e $W\left[\gamma_{R}\right] \equiv W_{R}$

Levando em consideração que o sistema está isolado do reservatório durante o intervalo $0 \leq t \leq \tau$, a Eq. 2-18 toma a seguinte forma: 


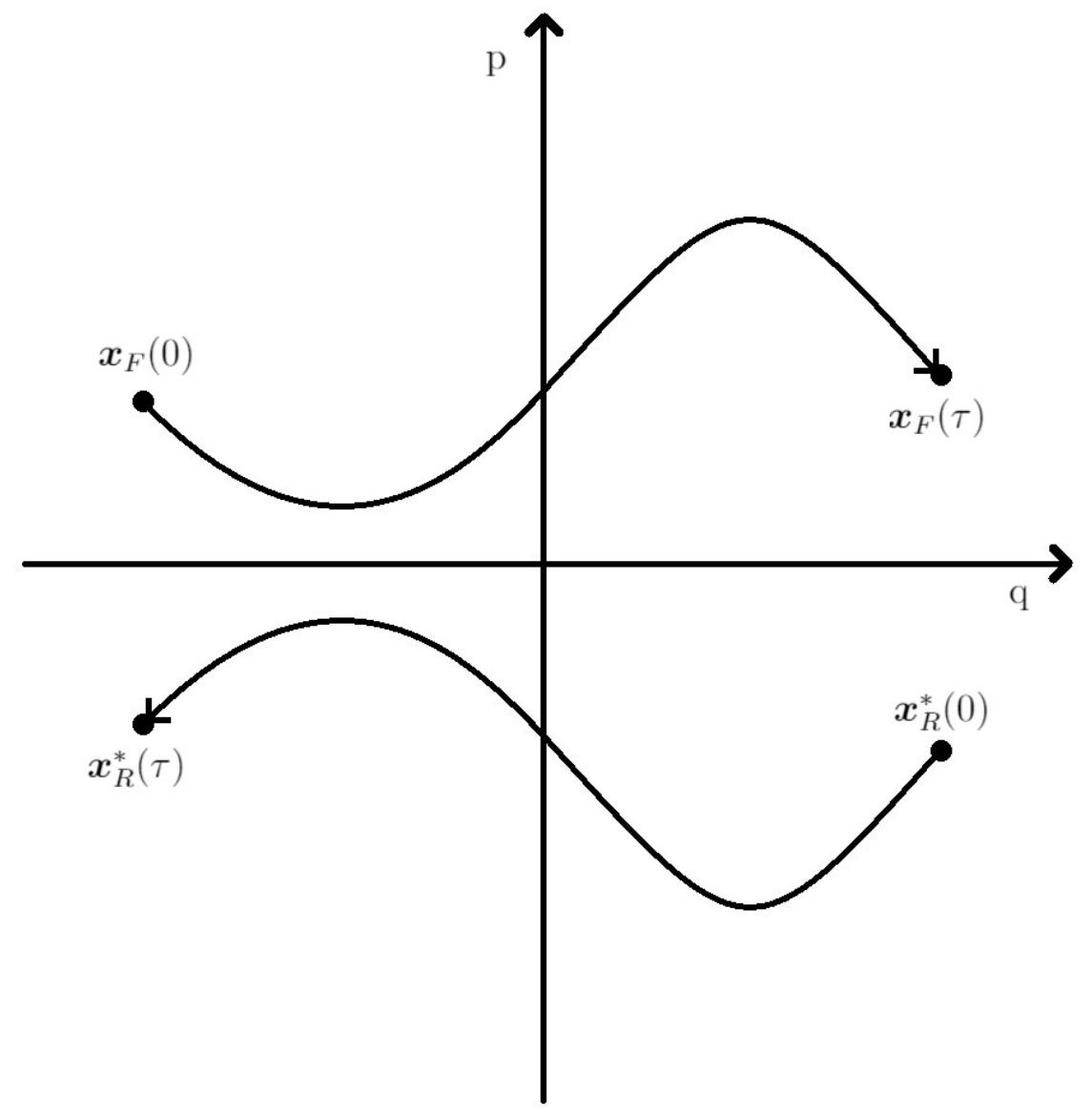

Figura 2.7: $\boldsymbol{x}^{*}$ são os estados microscópicos obtidos ao invertermos todos os momentos de $\boldsymbol{x}$.

$$
W=\mathcal{H}\left(\boldsymbol{x}_{\tau} ; B\right)-\mathcal{H}\left(\boldsymbol{x}_{\mathbf{0}} ; A\right) .
$$

A Hamiltoniana $\mathcal{H}(\boldsymbol{x} ; \lambda)$ para o sistema é determinística. Portanto, a probabilidade de realizarmos uma trajetória específica no espaço de fase depende somente da distibuição de probabilidade das condições iniciais em um estado de equilíbrio, dada pela Eq. (2-9). Porém, neste caso, devemos nos atentar que a função de partição é costruída somente com a Hamiltoniana do sistema, expressa pela Eq. (2-11). Daí podemos escrever a razão:

$$
\begin{aligned}
\frac{P_{F}\left[\gamma_{F}\right]}{P_{R}\left[\gamma_{R}\right]} & =\frac{Z_{B}}{Z_{A}} e^{\beta\left[H\left(\boldsymbol{x}_{R}(0) ; B\right)-\mathcal{H}\left(\boldsymbol{x}_{F}(0) ; A\right)\right]} \\
& =\frac{Z_{B}}{Z_{A}} e^{\beta\left[\mathcal{H}\left(\boldsymbol{x}_{F}(\tau) ; B\right)-\mathcal{H}\left(\boldsymbol{x}_{F}(0) A\right)\right]} \\
& =e^{\beta\left(W_{F}-\Delta F\right)}
\end{aligned}
$$


Na Eq. (2-36), a passagem da primeira para segunda linha se deve à igualdade $\mathcal{H}\left(\boldsymbol{x}_{R}(0) ; B\right)=\mathcal{H}\left(\boldsymbol{x}_{F}(\tau) ; B\right)$, oriunda da natureza determinística da Hamiltoniana $\mathcal{H}(\bullet)$. Já na passagem da segunda para a terceira linha, é utilizada a importante relação para o trabalho da Eq. (2-18) e a Eq. (2-27). A temperatura $T$ em $\beta=1 / k_{B} T$ é a temperatura inicial, que é a mesma no início de cada trajetória devido ao tempo concedido ao sistema para que entre em equilíbrio térmico com o reservatório.

A relação da Eq. (2-36) tem sido derivada na literatura para diversas configurações de sistemas $[47,38,48,49,1]$. Um resultado similar é o teorema de flutuação de Crooks [50]. Porém, enquanto a Eq. (2-36) é uma afirmação sobre as trajetórias, o teorema de flutuação de Crooks é uma afirmação sobre as distribuições dos valores do trabalho que satisfazem a equação:

$$
\frac{\rho_{F}(+W)}{\rho_{R}(-W)}=e^{\beta(W-\Delta F)}
$$

Os termos $\rho_{F}(W)$ e $\rho_{R}(W)$ na Eq. (2-37) são as distribuições para os valores do trabalho para a trajetória $\gamma_{F}$ e a trajetória reversa $\gamma_{R}$, respectivamente. Daí podemos concluir que o valor esperado do trabalho devido a distibuição $\rho_{F}(W)$ está localizado à direita do valor esperado do trabalho devido à distribuição $\rho_{R}(W)$, como ilustrado na Figura (2.8). Este teorema tem sido verificado por diversos experimentos na literatura [51, 52, 44, 43].

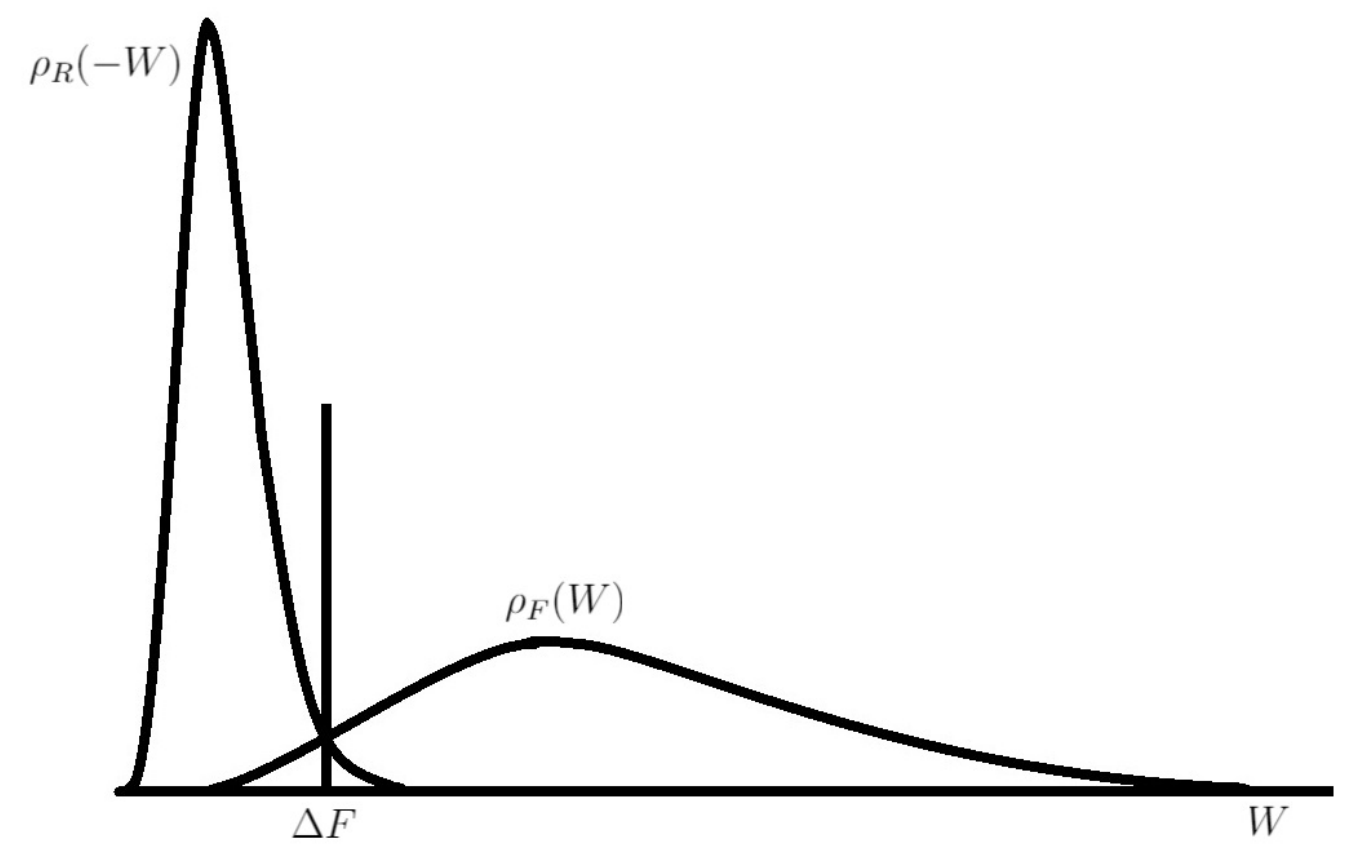

Figura 2.8: Distribuição para os valores do trabalho das trajetórias $\lambda_{F}$ e $\lambda_{R}$. O processo é reversível no ponto em que as curvas se interceptam, $W=\Delta F$.

É interessante notar que os valores esperados para o trabalho de cada 
distribuição $\left(\rho_{F}(W)\right.$ e $\left.\rho_{R}(-W)\right)$ corroboram a inequação (2-33). Porém, obtemos uma importante informação: eventualmente podemos observar um ciclo no qual o trabalho realizado sobre o sistema na trajetória $\gamma_{F}$ é menor que o realizado pelo sistema na trajetória reversa $\gamma_{R}$. Também podemos concluir que o processo é reversível no ponto $W=\Delta F$, no qual as curvas se interceptam. Ainda, se multiplicarmos ambos os lados da Eq. (2-37) pelo termo $\rho_{R}(-W) e^{-\beta W}$ e integrarmos sobre todo o espaço amostral, recuperamos a IJ expressa na Eq. (2-28) [46].

A Eq. 2-36 está estreitamente relacionada com o princípio de reversibilidade microscópica: qualquer sequência de eventos tem a mesma probabilidade do evento de tempo reverso [53]. Esta afirmação nos conduz à seguinte igualdade para processos reversíveis:

$$
\frac{P_{F}\left[\gamma_{F}\right]}{P_{R}\left[\gamma_{R}\right]}=1
$$

Portanto, para o caso de processos irreversíveis, a probabilidade da trajetória que observamos em um processo não é a mesma probabilidade do seu processo reverso, ou seja,

$$
P_{F}\left[\gamma_{F}\right] \neq P_{R}\left[\gamma_{R}\right]
$$

Daí a Eq. 2-36 pode ser vista como uma expansão do princípio de reversibilidade microscópica, já que reúne a Eq. 2-38 e a inequação (2-39) em uma única equação, estendendo a igualdade para sistemas conduzidos para fora do equilíbro pela variação de parâmetros externos.

\section{4}

\section{Entropia e trabalho dissipado}

Vamos utilizar uma definição de entropia que relaciona fortemente a Termodinâmica com a Teoria da Informação [54]:

$$
S=-k_{B} \int p(\boldsymbol{x}, \boldsymbol{v}, t) \ln p(\boldsymbol{x}, \boldsymbol{v}, t) d \boldsymbol{x} d \boldsymbol{v},
$$

na qual $p$ é a distribuição estatística do espaço de fase e a integral é realizada sobre todo espaço amostral de $p$. O termo

$$
I[p]=-\int p \ln p d \boldsymbol{x} d \boldsymbol{v}
$$

na Eq. (2-40) é conhecido como a entropia de informação associada a uma distribuição $p$. Se $p$ descrever um estado de equilíbrio termodinâmico, a entropia de informação coincide com a entropia termodinâmica multiplicada pela constante $k_{B}$. A Eq. (2-40) tem grande relevância no âmbito dos teoremas de flutuação porque relaciona uma informação microscópica $(I[p])$ com uma quantidade física mensurável $(S)$. 
Outra quantidade da Teoria da Informação que é relevante para este trabalho é a chamada entropia relativa, definida como [55]:

$$
D(p \| q)=\int p(\boldsymbol{x}, \boldsymbol{v}, t) \ln \left(\frac{p(\boldsymbol{x}, \boldsymbol{v}, t)}{q(\boldsymbol{x}, \boldsymbol{v}, t)}\right) d \boldsymbol{x} d \boldsymbol{v} \geq 0,
$$

na qual $p$ e $q$ são distribuições estatísticas e a integral é realizada sobre todo espaço amostral de $p . D(p \| q)$ é a medida da "distância" entre duas distribuições de probabilidade, $p$ e $q$. Quando $D(p \| q)=0$ as duas distribuições são iguais. Quanto maior o valor de $D(p \| q)$, menor a sobreposição das duas distribuições.

Para a Termodinâmica, a entropia relativa $D(p \| q)$ é útil para obter informações de processos irreversíveis, pois podemos mensurar o quanto um processo realizado em um sistema difere do seu processo reverso. Assim, as implicações da inequação (2-39) podem ser melhor detalhadas com mais uma informação, de modo que [56]:

$$
\begin{aligned}
D\left(P_{F}\left[\gamma_{F}\right] \| P_{R}\left[\gamma_{R}\right]\right) & =\int d \gamma_{F} P_{F}\left[\gamma_{F}\right] \ln \frac{P_{F}\left[\gamma_{F}\right]}{P_{R}\left[\gamma_{R}\right]} \\
& =\int d \gamma_{F} P_{F}\left[\gamma_{F}\right] \ln e^{\beta\left(W_{F}-\Delta F\right)} \\
& =\int d \gamma_{F} P_{F}\left[\gamma_{F}\right] \beta\left(W\left[\gamma_{F}\right]-\Delta F\right) \\
& =\beta\left(\langle W\rangle_{F}-\Delta F\right)=\beta\left\langle W_{\text {diss }}^{F}\right\rangle
\end{aligned}
$$

A passagem da primeira para a segunda linha na Eq. (2-43) é devido a relação da Eq. 2-36. O trabalho dissipado $\left\langle W_{\text {diss }}^{F}\right\rangle$ foi definido na Eq. (2-6). Neste caso, $\langle W\rangle_{F}$ é o valor esperado tomado pela distribuição dos valores do trabalho para a trajetória $\gamma_{F}$.

A Eq. (2-43) associa uma quantidade física no equilíbrio termodinâmico, $\left\langle W_{\text {diss }}^{F}\right\rangle$, a uma informação microscópica do sistema devido a sua trajetória. Podemos trocar as distribuições das trajetóris por um conjunto de parâmetros mais familiar: o conjunto de parâmetros do espaço de fase. Podemos definir a função $f_{F}(\boldsymbol{x}, t)$ como a densidade do espaço de fase que descreve a evolução do sistema durante a trajetória $\gamma_{F}$. Do mesmo modo, $f_{R}\left(\boldsymbol{x}^{*}, \tau-t\right)$ é a densidade do espaço de fase para o processo reverso $\left(\gamma_{R}\right)$, na qual $\boldsymbol{x}^{*}$ são os estados microscópicos obtidos ao invertermos todos os momentos de $\boldsymbol{x}$. Podemos expressar a função de densidade do espaço de fase na forma [57]:

$$
f(\boldsymbol{x}, t)=\left\langle\delta\left[\boldsymbol{x}-\boldsymbol{x}_{k}(t)\right]\right\rangle,
$$

na qual $\boldsymbol{x}_{1}(t), \boldsymbol{x}_{2}(t) \ldots \boldsymbol{x}_{k}(t)$ são as trajetórias descritas pela distribuição do espaço de fase. O valor esperado do lado direito da Eq. (2-44) é tomado sobre 
a distribuição de probabilidade das trajetórias $\boldsymbol{x}_{k}(t)$.

As densidades $f_{F}\left(\boldsymbol{x}, t_{1}\right)$ e $f_{R}\left(\boldsymbol{x}^{*}, \tau-t_{1}\right)$ são registros instantâneos no tempo $t=t_{1}$ dos estados no espaço de fase do sistema durante cada processo. Estes registros são tomados conforme os parâmetros para o trabalho, $\lambda_{R}$ e $\lambda_{F}$, alcançam o valor $\lambda_{F}\left(t_{1}\right)=\lambda_{R}\left(\tau-t_{1}\right)$. Neste momento os valores das densidades $f_{F}\left(\boldsymbol{x}, t_{1}\right)$ e $f_{R}\left(\boldsymbol{x}^{*}, \tau-t_{1}\right)$ são diferentes, isto é, os estados microscópicos são diferentes quando o parâmetro do trabalho assume o mesmo valor em ambas as trajetórias. A entropia relativa para as duas distribuições é [58]:

$$
D\left(f_{F} \| f_{R}\right) \leq \beta\left\langle W_{d i s s}^{F}\right\rangle .
$$

Combinando o último resultado com a Eq. (2-43), temos

$$
D\left(f_{F} \| f_{R}\right) \leq \frac{P_{F}\left[\gamma_{F}\right]}{P_{R}\left[\gamma_{R}\right]}=\beta\left\langle W_{d i s s}^{F}\right\rangle
$$

Daí podemos concluir que a irreversibilidade de um processo pode ser expressa por uma inequação que relaciona duas distribuições de probabilidade, tanto no espaço das trajetórias quanto no espaço de fase. A igualdade na Eq. (2-46) é válida quando o sistema é isolado termicamente do reservatório, enquanto o parâmetro do trabalho varia para cada processo.

Os últimos resultados são relacionado ao trabalho dissipativo macroscópico. Podemos ainda obter um resultado importante para o trabalho dissipativo microscópico utilizando a IJ. Ao substituirmos o termo $W=W_{\text {diss }}+\Delta F$ na Eq. (2-27) obtemos a igualdade:

$$
\left\langle e^{-\beta W_{\text {diss }}}\right\rangle=1
$$

A Eq. (2-47) contém uma importante informação: para qualquer protocolo do parâmetro do trabalho $\lambda(t)$, o valor esperado para a quantidade $e^{-\beta W_{\text {diss }}}$ é o mesmo, inclusive para processos que levem o sistema para muito longe dos estados de equilíbrio (tal como um gás sendo comprimido quase instantaneamente).

\section{5}

\section{Equivalência de ensembles}

Demonstrada uma das possíveis derivações da IJ, uma interessante elucubração que pode ser feita é: se tomássemos as condições iniciais de uma distribuição microcanônica teríamos o mesmo resultado para a IJ?

Tomando um resultado da Eq.(2-23), vamos relacionar a expressão com o ensemble microcanônico da seguinte forma [59]: 


$$
\begin{aligned}
\left\langle e^{-\beta W}\right\rangle^{c a n} & \left.=\int d \boldsymbol{x}_{\mathbf{0}} d \boldsymbol{y}_{\mathbf{0}} \frac{1}{Z_{A}} \exp \left[-\beta\left\{\boldsymbol{H}\left(\boldsymbol{x}_{\mathbf{0}}, \boldsymbol{y}_{\mathbf{0}} ; \lambda=A\right)\right]+\beta \tilde{W}\left(\boldsymbol{x}_{\mathbf{0}}, \boldsymbol{y}_{\mathbf{0}}\right)\right\}\right] \\
& =\int d \boldsymbol{x}_{\mathbf{0}} d \boldsymbol{y}_{\mathbf{0}} \frac{1}{Z_{A}} \int d E \delta\left(\boldsymbol{H}\left(\boldsymbol{x}_{\mathbf{0}}, \boldsymbol{y}_{\mathbf{0}} ; \lambda=A\right)-E\right) \times \\
& \left.\times \exp \left[-\beta\left\{\boldsymbol{H}\left(\boldsymbol{x}_{\mathbf{0}}, \boldsymbol{y}_{\mathbf{0}} ; \lambda=A\right)\right]+\beta \tilde{W}\left(\boldsymbol{x}_{\mathbf{0}}, \boldsymbol{y}_{\mathbf{0}}\right)\right\}\right] \\
& =\frac{1}{Z_{A}} \int d E \omega_{A}(E) e^{-\beta E}\left\langle e^{-\beta W}\right\rangle_{E}^{m i c} \\
& =\frac{1}{Z_{A}} \int d E \exp \left[-\beta E+S_{A}(E) / k_{\beta}+\ln \left\langle e^{-\beta W}\right\rangle_{E}^{m i c}\right]
\end{aligned}
$$

Na qual $\left\langle e^{-\beta W}\right\rangle^{c a n}$ é o valor esperado da quantidade $e^{-\beta W}$ tomado pela distribuição canônica, $\omega_{A}(E)$ é o número de estados microscópico no volume $\boldsymbol{H}\left(\boldsymbol{x}_{\mathbf{0}}, \boldsymbol{y}_{\mathbf{0}} ; \lambda=A\right), S_{A}(E)$ é a entropia da Hamiltoniana $\boldsymbol{H}$ à energia $E$ e parâmetro do trabalho em $\lambda=A$ e $\left\langle e^{-\beta W}\right\rangle_{E}^{m i c}$ é o valor esperado da quantidade $e^{-\beta W}$ tomado pela distribuição microcanônica com a energia fixa em $E$. A conhecida relação para a entropia [60],

$$
S=k_{\beta} \ln \Omega,
$$

foi utilizada para obter $S_{A}(E)$, na qual $\Omega$ é o volume dos estados microscópicos de todo o espaço de fase.

A integral sobre $E$ da Eq. (2-48) é dominado pelo valor de $E$ que maximiza o integrando e satisfaz a equação:

$$
-\beta+\frac{\partial\left(S_{A}(E) / k_{\beta}\right)}{\partial E}+\frac{\partial \ln \left\langle e^{-\beta W}\right\rangle_{E}^{m i c}}{\partial E}=0 .
$$

O trabalho $W$ é obtido pela manipulação do sistema de interesse, e possui muito menos graus de liberdade do que o reservatório térmico. Portanto, a escala de variação de energia do trabalho deve ser muito menor do que o do sistema e reservatório combinados $\left(S_{A}(E)\right)$. Daí podemos negligenciar a contribuição de $W$ para Eq. (2-50) e concluir que o valor de energia que maximiza a equação é o valor da energia do sistema de interesse e reservatório combinados no equilíbrio (à temperatura $T$ ).

Denotando como $\tilde{e}$ o valor de $E$ que satisfaz a Eq. (2-50), podemos escrever $Z_{A}=\exp \left[-\beta \tilde{e}+S_{A}(\tilde{e}) / k_{\beta}\right]$. Assim, desconsiderando as correções pouco significativas, obtemos a equivalência:

$$
\left\langle e^{-\beta W}\right\rangle^{c a n}=\left\langle e^{-\beta W}\right\rangle_{E}^{m i c} .
$$

\section{6}

\section{Características do modelo}

Neste capítulo descrevemos o modelo para o qual relacionamos quantidades microscópicas fora do equilíbrio com quantidades macroscópicas no 
equilíbrio. Ainda que facilmente notável, não explicitamos duas importantes premissas. A primeira é que não consideramos os efeitos quânticos. Logo, estes efeitos podem ser ignorados para o caso proposto. A segunda premissa é que o sistema e o reservatório são isolados de qualquer outro grau de liberdade externo.

A abordagem utlizada para modelar o sistema proposto foi por meio de uma Hamiltoniana. Porém, as relações obtidas para os sistemas microscópicos fora do equilíbrio não dependem da dinâmica pela qual o sistema é modelado. Estas relações tem sido derivadas por diferentes abordagens [61, 39, 62, 40].

Embora as relações encontradas não dependam da abordagem, quando analisamos sistemas fora do equilíbrio devemos especificar quais abordagens estamos utilizando. Isto se deve ao fato de que a escolha de cada abordagem encerra algumas suposições. Nos capítulos posteriores, vamos modelar o sistema através de uma equação de Langevin. Assim, o reservatório térmico será tratado de forma implícita nessa equação. 


\section{3 \\ Modelo Fourier-Laplace}

Para solucionarmos as equações que modelam os sistemas de interesse deste trabalho, vamos utilizar um método que toma a definição da transformada de Laplace como um elemento central para a simplificação das operações matemáticas necessárias. Neste capítulo, explicitamos este método através do cálculo da distribuição de probabilidade para a posição e a velocidade de uma partícula browniana no equilíbrio. Em seguida, vamos calcular a função geradora de probabilidade do trabalho fora do equilíbrio para uma partícula Browniana sob a ação de um potencial harmônico [63]. Também verificamos a IJ para o modelo. Os procedimentos realizados originalmente para os cálculos, e a definição dos dois sistemas, foram publicados por Soares-Pinto \& Morgado, e podem ser encontrados em [64, 65].

\section{1}

\section{Definição do modelo para o cálculo da distribuição de probabilidade estacionária}

O modelo que vamos utilizar para aplicar o método é o de uma partícula Browniana sujeita a um potencial harmônico e uma força de amortecimento proporcional à velocidade $v(t)$. Sobre a partícula também atua uma força estocástica representada na equação pelo ruído colorido Gaussiano $\eta(t)$ [66]. É assumido que a transformada de Laplace de $\eta(t)$ possa ser calculada [13]. Neste modelo, o reservatório térmico é representado implicitamente por $\eta(t)$. A equação que descreve a evolução temporal do sistema é

$$
m \dot{v}(t)=-\gamma v(t)-k x(t)+\eta(t) .
$$

Na qual $v(t)=\dot{x}(t), k$ é a constante elástica de uma mola e $\gamma$ é o coeficiente de fricção. Os termos $v(0)=v_{0}$ e $x(0)=x_{0}$ são as condições iniciais para o sistema no equilíbrio.

Utilizando a definição de $\dot{x}(t)$, podemos expressar $x(t)=x_{0}+\int_{0}^{t} v\left(t^{\prime}\right) d t^{\prime}$. Substituindo $x(t)$ na Eq. 3-1, obtemos

$$
m v(t)=m v_{0}-\gamma \int_{0}^{t} v\left(t^{\prime}\right) d t^{\prime}-k x_{0} t-k \int_{0}^{t} \int_{0}^{t^{\prime}} v\left(t^{\prime \prime}\right) d t^{\prime \prime} d t^{\prime}+\int_{0}^{t} \eta\left(t^{\prime}\right) d t^{\prime} .
$$


Tomando a transformada de Laplace de $x(t)=x_{0}+\int_{0}^{t} v\left(t^{\prime}\right) d t^{\prime}$ e da Eq. 3 -2, obtemos as expressões da transformada de $x(t)$ e $v(t)$, como segue:

$$
\tilde{x}(s)=A(s)+B(s) \tilde{\eta}(s)
$$

e

$$
\tilde{v}(s)=C(s)+D(s) \tilde{\eta}(s)
$$

Nas quais,

$$
\begin{aligned}
& A(s)=\frac{(s+\beta) x_{0}+v_{0}}{s(s+\beta)+w_{0}^{2}}, \\
& B(s)=\frac{m^{-1}}{s(s+\beta)+w_{0}^{2}}, \\
& C(s)=\frac{s v_{0}-w_{0}^{2} x_{0}}{s(s+\beta)+w_{0}^{2}}, \\
& D(s)=\frac{m^{-1} s}{s(s+\beta)+w_{0}^{2}},
\end{aligned}
$$

na qual $w_{0}=k / m$ e $\beta=\gamma / m$. Os cálculos explícitos podem ser verificados no apêndice B.1.

No âmbito dos teoremas ergódicos, vamos definir a distribuição de probabilidade estacionária para o espaço de fase do sistema, considerando o teorema ergódico de Birkhoff [67]. Para o modelo proposto, temos:

$$
p_{e q}(x, v)=\lim _{T \rightarrow \infty} \frac{1}{T} \int_{0}^{T} d t p(x, v, t)=\lim _{T \rightarrow \infty} \frac{1}{T} \int_{0}^{T} d t\langle\delta[x-x(t)] \delta[v-v(t)]\rangle,
$$

na qual a função $p(\bullet)$ é a densidade do espaço de fase definida na Eq. (2-44), $x(t)$ e $v(t)$ são soluções da Eq. (3-2) e o valor esperado $\langle\bullet\rangle$ é tomado sobre a distribuição de $\eta(t)$.

Façamos a substituição $z=1 / T$ na Eq. (3-9). Para sistemas ergódicos que satisfaçam a condição $g(t \rightarrow \infty)=\lim _{s \rightarrow 0} s \tilde{g}(s)=L$, na qual $\tilde{g}(s)$ é a transformada de Laplace de $g(t)$, o teorema do valor final nos permite escrever [68]:

$$
p_{e q}(x, v)=\lim _{z \rightarrow 0} z \int_{0}^{\infty} d t e^{-z t}\langle\delta[x-x(t)] \delta[v-v(t)]\rangle_{\eta} .
$$

Ainda temos uma terceira abordagem para obtermos a distribuição estacionária de $p(x, v, t)$, que segue:

$$
p_{e q}(x, v)=\lim _{t \rightarrow \infty} p(x, v, t) .
$$

Esta última definição toma em consideração a característica de sistemas em equilíbrio termodinâmico, ou seja, a descrição de estados estáticos destes sistemas [21].

Para demonstrarmos algumas técnicas utilizadas no restante deste tra- 
balho, nesta seção vamos utilizar a identidade da Eq. (3-10). Assim, realizando um procedimento similar ao demonstrado no apêndice B.2, podemos expressar a evolução temporal da distribuição de probabilidade para a posição e velocidade na forma:

$$
\begin{aligned}
p(x, v, t) & =\langle\delta[x-x(t)] \delta[v-v(t)]\rangle_{\eta} \\
& =\lim _{\epsilon \rightarrow 0} \sum_{m, n=0}^{\infty} \frac{1}{m !} \frac{1}{n !} \int_{-\infty}^{\infty} \int_{-\infty}^{\infty} \frac{d Q}{2 \pi} \frac{d P}{2 \pi}(-i Q)^{m}(-i P)^{n} e^{i Q x+i P v} \times \\
& \times \int_{-\infty}^{\infty} \int_{-\infty}^{\infty} \prod_{i=1}^{m} \frac{d q_{i}}{2 \pi} \prod_{j=1}^{n} \frac{d p_{j}}{2 \pi} e^{\sum_{f=1}^{m}\left(i q_{f}+\epsilon\right) t+\sum_{h=1}^{n}\left(i p_{h}+\epsilon\right) t} \times \\
& \times\left\langle\prod_{i=1}^{m} \tilde{x}\left(i q_{i}+\epsilon\right) \prod_{j=1}^{n} \tilde{v}\left(i p_{j}+\epsilon\right)\right\rangle_{\eta} .
\end{aligned}
$$

Substituindo o resultado da Eq. (3-12) na identidade definida na Eq. (310), e realizando a integral sobre o tempo $t$, obtemos a distribuição estacionária do espaço de fase:

$$
\begin{aligned}
p(x, v) & =\lim _{z, \epsilon \rightarrow 0} \sum_{l, m=0}^{\infty} \frac{1}{m !} \frac{1}{n !} \int_{-\infty}^{\infty} \int_{-\infty}^{\infty} \frac{d Q}{2 \pi} \frac{d P}{2 \pi}(-i Q)^{m}(-i P)^{n} e^{i Q x+i P v} \times \\
& \times \int_{-\infty}^{\infty} \int_{-\infty}^{\infty} \prod_{i=1}^{m} \frac{d q_{i}}{2 \pi} \prod_{j=1}^{n} \frac{d p_{j}}{2 \pi} \frac{z}{z-\left[\sum_{f=1}^{m}\left(i q_{f}+\epsilon\right)+\sum_{h=1}^{n}\left(i p_{h}+\epsilon\right)\right]} \times \\
& \times\left\langle\prod_{i=1}^{m} \tilde{x}\left(i q_{i}+\epsilon\right) \prod_{j=1}^{n} \tilde{v}\left(i p_{j}+\epsilon\right)\right\rangle_{\eta} .
\end{aligned}
$$

O último resultado pode fazer parecer que o cálculo da distribução de probabilidade se tornou mais complicado, porém, as próximas considerações irão demonstrar que as integrais da Eq. (3-13) serão reduzidas a somente algumas contribuições não nulas.

\subsection{1}

\section{Considerações sobre o ruído colorido $\eta(t)$}

Para o nosso modelo, o processo estocástico conduzido pelo ruído colorido Gaussiano $\eta(t)$ é definido a seguir [66]:

$$
\begin{aligned}
\langle\eta(t)\rangle & =0 \\
\left\langle\eta(t) \eta\left(t^{\prime}\right)\right\rangle & =\frac{D}{2 \tau} e^{\frac{-\left|t-t^{\prime}\right|}{\tau}} .
\end{aligned}
$$


Na qual $D$ e $\tau$ são constantes com unidade de tempo. Quando $\tau \rightarrow 0$, o ruído $\eta(t)$ é reduzido a um ruído branco.

Nós assumimos uma forma simples de dissipação, na qual o teorema de flutuação-dissipação não se aplica [69]. Se o sistema está fora do equilíbrio, esta consideração é válida e, portanto, o núcleo de memória de dissipação e as propriedades do ruído podem ser consideradas independentes [70].

Devido à natureza Gaussiana do ruído $\eta(t)$, o segundo cumulante é o único cumulante não nulo [66]. Daí, pelo teorema de Wick, podemos escrever [71]:

$$
\left\langle\prod_{i=1}^{l} \eta\left(t_{i}\right)\right\rangle=\sum^{2 a 2}\left\langle\eta\left(t_{i_{1}}\right) \eta\left(t_{i_{2}}\right)\right\rangle \ldots\left\langle\eta\left(t_{i_{l-1}}\right) \eta\left(t_{i_{l}}\right)\right\rangle,
$$

na qual $\sum^{2 a 2}$ representa a soma sobre todas as possíveis combinações par a par. Para $l=2 p$, teremos $\frac{(2 p) !}{2^{p} p !}$ combinações dos pares de funções de $\eta$.

A transformada de Laplace do segundo cumulante é

$$
\begin{aligned}
\left\langle\tilde{\eta}\left(i q_{i}+\epsilon\right) \tilde{\eta}\left(i q_{j}+\epsilon\right)\right\rangle & =\frac{D \tau}{2\left(\left(i q_{i}+\epsilon\right) \tau+1\right)\left(\left(i q_{j}+\epsilon\right) \tau+1\right)}+ \\
& +\frac{D}{\left(\left(i q_{i}+i q_{j}+2 \epsilon\right)\right)\left(\left(i q_{i}+\epsilon\right) \tau+1\right)\left(\left(i q_{j}+\epsilon\right) \tau+1\right)}
\end{aligned}
$$

Os cálculos realizados para obtenção da transformada de Laplace do ruído podem ser conferidos no apêncice C.3. A Eq. (3-17) é reduzida ao ruído branco quando $\tau \rightarrow 0$.

\subsection{2}

Irreversibilidade $(z \rightarrow 0)$

O denominador em comum nos termos $A(s), B(s), C(s)$ e $D(s)$ das Eqs. (3-3) e (3-4) é a relação de dispersão do oscilador harmônico amortecido. Os polos são:

$$
\kappa_{ \pm}=-\frac{\beta}{2} \pm i \frac{1}{2} \sqrt{4 w_{0}^{2}-\beta^{2}}
$$

na qual é assumido que $4 w_{0}^{2}-\beta^{2}>0$. É possível observar que, diferentemente de $B(s)$ e $D(s)$, os termos $A(s)$ e $C(s)$ possuem informações relacionadas às condições iniciais. Por outro lado, $B(s)$ e $D(s)$ estão relacionadas com a parte estocástica da dinâmica do modelo. Logo, o sistema conservará a memória das condições inicias na medida que os termos $A(s)$ e $C(s)$ contribuam para o resultado final. 
A distribuição de equilíbrio (estacionária) da Eq. (3-13) corresponde a uma soma de infinitos produtos de funções polinomiais com os polos dados pela Eq. (3-18). Vamos denominar o único termo propagador que contém o efeito do tempo de $G(z)$, expresso a seguir:

$$
G(z)=\frac{z}{z-\left[\sum_{f=1}^{m}\left(i q_{f}+\epsilon\right)+\sum_{h=1}^{n}\left(i p_{h}+\epsilon\right)\right]} .
$$

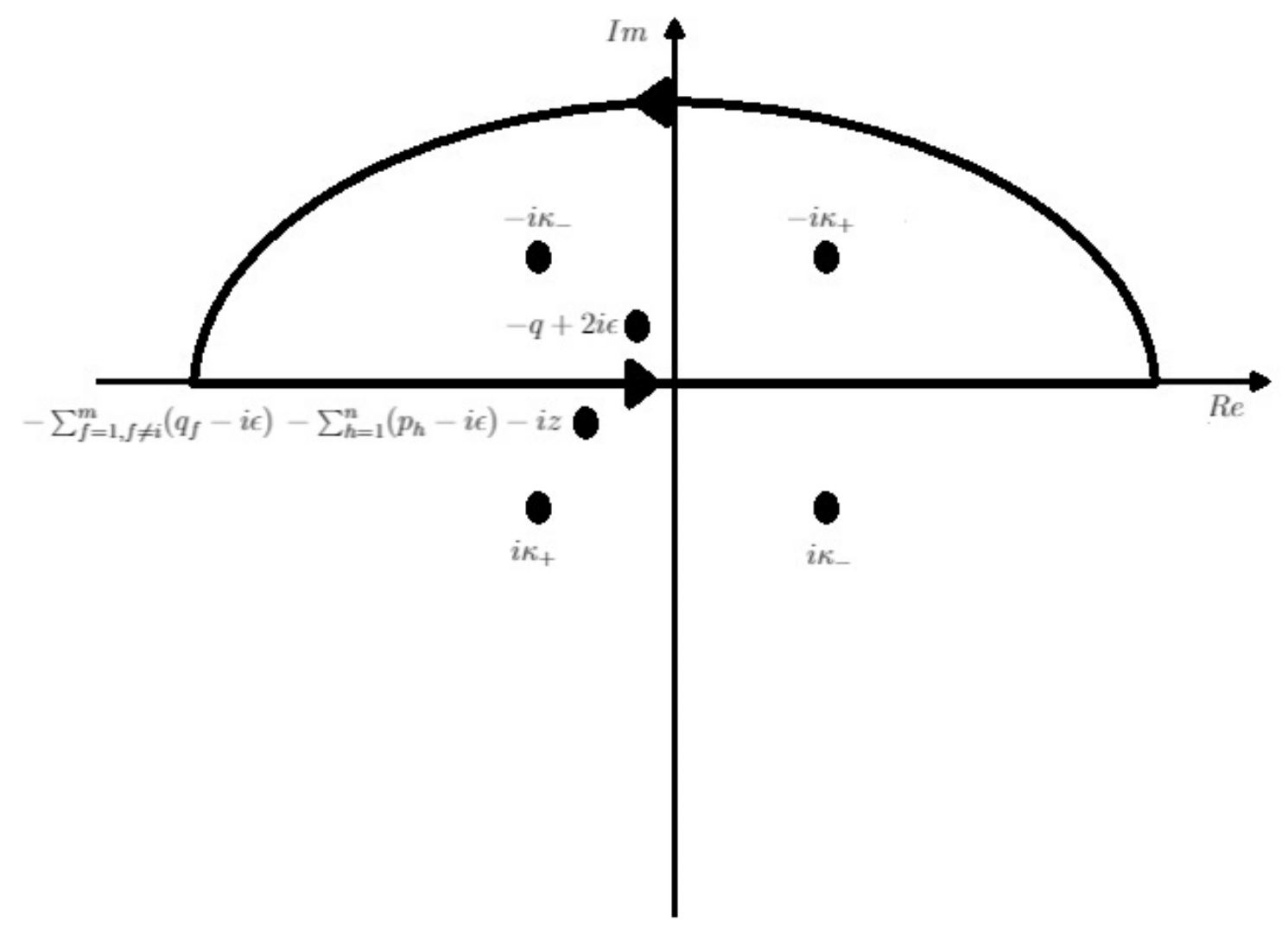

Figura 3.1: Caminho da integração para as variáveis $p$ e $q$.

Para facilitar os cálculos, vamos agora analisar as propriedades do propagador $\mathrm{G}(\mathrm{z})$. Ao calcularmos as integrais das variáveis $q_{i}$ e $p_{j}$, escolhendo o caminho superior da Figura 3.1, os polos associados ao propagador $\left(q_{i}=\right.$ $-\sum_{f=1, f \neq i}^{m}\left(q_{f}-i \epsilon\right)-\sum_{h=1}^{n}\left(p_{h}-i \epsilon\right)-i z$ e $p_{j}=-\sum_{f=1}^{m}\left(q_{f}-i \epsilon\right)-\sum_{h=1, h \neq j}^{n}\left(p_{h}-\right.$ $i \epsilon)-i z)$ ficam foram do contorno de integração. Portanto, estes polos não contribuem para os cálculos dos resíduos. Contudo, ao calcularmos os resíduos não nulos dos polos dentro do contorno (Eq. (3-18)), os valores associados a estes polos são adicionados ao denominador de $\mathrm{G}(\mathrm{z})$. Daí surge a relevância de analisarmos o limite $z \rightarrow 0$ de $G(z)$.

Quando o denominador do propagador $G(z)$ é diferente de zero, temos $\lim _{z \rightarrow 0} G(z)=0$. Todavia, quando a soma das variáveis $q_{i}$ e $p_{j}$ é eliminada 
do denominador, o único valor resultante do limite será $\lim _{z \rightarrow 0} G(z)=1$ e as integrais contribuirão para o cálculo.

Os termos $A(s)$ e $C(s)$ não possuem relação com o ruído $\eta(t)$. Logo, os resíduos associados a eles serão calculados ao redor dos polos da relação de dispersão expressados na Eq. (3-18). Ao procedermos deste modo, os valores dos polos $q_{i}$ e $p_{j}$ serão adicionados ao denominador de $G(z)$. Ao final das integrações sobre os polos de $A(s)$ e $C(s)$, um valor não nulo será obtido da soma $i\left(q_{1}+q_{2}+\ldots+q_{m}+p_{1}+p_{2}+\ldots+p_{n}\right)+(m+n) \epsilon$. Então, quando aplicarmos o limite $z \rightarrow 0$, este procedimento resultará em nenhuma contribuição para o cálculo.

Assim, podemos concluir que este é o modo no qual a irreversibilidade se manifesta matematicamente na direção do equilíbrio termodinâmico: a memória das condições iniciais é perdida ao realizarmos as transformadas de Laplace inversas.

\subsection{3}

\section{Integrais relevantes}

Levando em consideração a análise das duas últimas seções, podemos concluir que as únicas integrais que contribuem para o resultado final são as que possuem os termos $B(s) B(s), D(s) D(s)$ e $B(s) D(s)$. Vamos expressar somente os resultados após os cálculos dos resíduos. Uma demonstração de como os cálculos são realizados pode ser conferida no apêndice (B.3).

\subsubsection{1}

\section{Contribuição de $B(s) B(s)$}

Substituindo $\tilde{x}(s)$ e $\tilde{v}(s)$ na Eq. (3-13), a contribuição típica para a distribuição de equilíbrio dada pela posição é calculada integrando sobre o contorno da Figura 3.1 e é expressa na forma:

$$
\begin{aligned}
& \int_{-\infty}^{\infty} \int_{-\infty}^{\infty} \frac{d q_{i}}{2 \pi} \frac{d q_{j}}{2 \pi} \frac{z}{z-i\left(q_{i}+q_{j}+\square\right)} B\left(i q_{i}+\epsilon\right) B\left(i q_{j}+\epsilon\right)\left\langle\eta\left(i q_{i}+\epsilon\right) \eta\left(i q_{j}+\epsilon\right)\right\rangle= \\
= & \frac{z}{z-i \boxminus 2\left(m+k \tau^{2}\right)^{2}-\tau^{2} \gamma^{2}}\left(\frac{m^{2}}{k \gamma}-\frac{\tau^{2} \gamma}{k}+\frac{\tau^{2} m}{\gamma}+\tau^{3}\right) . \\
& \text { O símbolo } \square \text { respresenta todos os termos que não estão sob integração. }
\end{aligned}
$$
Ao final do cálculo de todos os resíduos, ele será nulo. De acordo com a Eq. (3-16), a contribuição do termo $B(s) B(s)$ é a soma das combinações 2 a 2 que podemos formar. Assim, para $m=2 \theta$, temos: 


$$
\frac{(2 \theta) !}{2^{\theta} \theta !}\left[\frac{D}{2\left(m+k \tau^{2}\right)^{2}-\tau^{2} \gamma^{2}}\left(\frac{m^{2}}{k \gamma}-\frac{\tau^{2} \gamma}{k}+\frac{\tau^{2} m}{\gamma}+\tau^{3}\right)\right]^{\theta}
$$

\subsubsection{2}

\section{Contribuição de $D(s) D(s)$}

De modo similar ao termo $B(s) B(s)$, podemos expressar a contribuição de $D(s) D(s)$ na forma:

$$
\begin{aligned}
& \int_{-\infty}^{\infty} \int_{-\infty}^{\infty} \frac{d p_{i}}{2 \pi} \frac{d p_{j}}{2 \pi} \frac{z}{z-i\left(p_{i}+p_{j}+\square\right)} D\left(i p_{i}+\epsilon\right) D\left(i p_{j}+\epsilon\right)\left\langle\eta\left(i p_{i}+\epsilon\right) \eta\left(i p_{j}+\epsilon\right)\right\rangle= \\
= & \frac{z}{z-i \sqsubseteq} \frac{-D}{2\left(m+k \tau^{2}\right)^{2}-\tau^{2} \gamma^{2}}\left(-\frac{m}{\gamma}-\frac{\tau^{2} k}{\gamma}+\tau\right) .
\end{aligned}
$$

Assim, quando $z \rightarrow 0$, a contribuição das médias da velocidade para a distribuição de probabilidade é dada por:

$$
\frac{(2 \alpha) !}{2^{\alpha} \alpha !}\left[\frac{-D}{2\left(m+k \tau^{2}\right)^{2}-\tau^{2} \gamma^{2}}\left(-\frac{m}{\gamma}-\frac{\tau^{2} k}{\gamma}+\tau\right)\right]^{\alpha},
$$

para $n=2 \alpha$.

\subsubsection{3}

\section{Contribuição de $B(s) D(s)$}

Por último, a contribuição cruzada da posição e velocidade pode ser expressa na seguinte forma:

$$
\begin{aligned}
& \int_{-\infty}^{\infty} \int_{-\infty}^{\infty} \frac{d q_{i}}{2 \pi} \frac{d p_{j}}{2 \pi} \frac{z}{z-i\left(q_{i}+p_{j}+\square\right)} B\left(i q_{i}+\epsilon\right) D\left(i p_{j}+\epsilon\right)\left\langle\eta\left(i q_{i}+\epsilon\right) \eta\left(i p_{j}+\epsilon\right)\right\rangle= \\
= & \frac{z}{z-i \cdot} \int_{-\infty}^{\infty} \frac{d p_{j}}{2 \pi} \frac{m^{-2} D p_{j}}{\left(p_{j}+i \kappa_{+}\right)\left(p_{j}+i \kappa_{-}\right)\left(p_{j}-i \kappa_{+}\right)\left(p_{j}-i \kappa_{-}\right)}=0 .
\end{aligned}
$$

A última integral é nula por causa do polinônimo de potência ímpar no numerador. Podemos interpretar esta nulidade pela falta de relação entre a energia cinética e a energia potencial do sistema no equilíbrio. Um exemplo do contrário seria uma partícula sob a ação de um potencial magnético, pois neste caso existe uma relação entre o momento da partícula e o potencial no qual está submetida. Fora do equilíbrio, este termo da distribuição não é nulo. Uma amostra de como se dá esta relação pode ser conferida de forma exata em [72]. Como o termo cruzado não contribui para a distribuição, podemos expressar esta última como o produto do termo da posição e da velocidade: 


$$
p_{e q}(x, v)=p_{e q}(x) p_{e q}(v)
$$

\subsection{4}

\section{Distribuição de probabilidade para a posição e velocidade no equilíbrio termodinâmico}

Coletando os resultados obtidos das integrais relevantes para a composição da distribuição de probabilidade, podemos construir a seguinte expressão para a Eq. 3-13:

$$
\begin{aligned}
p_{e q}(x, v) & =\sum_{\theta=0}^{\infty} \sum_{\alpha=0}^{\infty} \int_{-\infty}^{\infty} \int_{-\infty}^{\infty} \frac{d Q}{2 \pi} \frac{d P}{2 \pi} e^{i Q x+i P v} \times \\
& \times \frac{(-i Q)^{2 \theta}}{2 \theta !} \frac{(2 \theta) !}{2^{\theta} \theta !}\left[\frac{D}{2\left(m+k \tau^{2}\right)^{2}-\tau^{2} \gamma^{2}}\left(\frac{m^{2}}{k \gamma}-\frac{\tau^{2} \gamma}{k}+\frac{\tau^{2} m}{\gamma}+\tau^{3}\right)\right]^{\theta} \times \\
& \times \frac{(-i P)^{2 \alpha}}{2 \alpha !} \frac{(2 \alpha) !}{2^{\alpha} \alpha !}\left[\frac{m}{2\left(m+k \tau^{2}\right)^{2}-\tau^{2} \gamma^{2}}\left(\frac{m}{\gamma}+\frac{\tau^{2} k}{\gamma}-\tau\right)\right]^{\alpha} \\
& =\int_{-\infty}^{\infty} \frac{d Q}{2 \pi} \exp \left[i Q x-\frac{D Q^{2}}{4\left(m+k \tau^{2}\right)^{2}-2 \tau^{2} \gamma^{2}}\left(\frac{m^{2}}{k \gamma}-\frac{\tau^{2} \gamma}{k}+\frac{\tau^{2} m}{\gamma}+\tau^{3}\right)\right] \times \\
& \times \frac{d P}{2 \pi} \exp \left[i P v-\frac{m}{4\left(m+k \tau^{2}\right)^{2}-2 \tau^{2} \gamma^{2}}\left(\frac{m}{\gamma}+\frac{\tau^{2} k}{\gamma}-\tau\right)\right] \\
& =\sqrt{\frac{\left(m+k \tau^{2}\right)^{2}-\tau^{2} \gamma^{2}}{\pi D\left(\frac{m^{2}}{k \gamma}-\frac{\tau^{2} \gamma}{k}+\frac{\tau^{2} m}{\gamma}+\tau^{3}\right)} \exp \left[-\frac{m^{2}\left[\left(1+\tau^{2} \omega_{0}^{2}\right)^{2}-\tau^{2} \beta^{2}\right] x^{2}}{D\left(\frac{m^{2}}{k \gamma}-\frac{\tau^{2} \gamma}{k}+\frac{\tau^{2} m}{\gamma}+\tau^{3}\right)}\right] \times} \\
& \times \sqrt{\frac{\left(m+k \tau^{2}\right)^{2}-\tau^{2} \gamma^{2}}{\pi D\left(\frac{m}{\gamma}+\frac{\tau^{2} k}{\gamma}-\tau\right)} \exp \left[-\frac{m^{2}\left[\left(1+\tau^{2} \omega_{0}^{2}\right)^{2}-\tau^{2} \beta^{2}\right] v^{2}}{D\left(\frac{m}{\gamma}+\frac{\tau^{2} k}{\gamma}-\tau\right)}\right]} .
\end{aligned}
$$

A Eq.3-26 é a distribuição estacionária analítica no equilíbrio para a posição e a velocidade da partícula Browniana do sistema modelado. Quando $\tau \rightarrow 0$, temos a distribuição para a partícula quando esta está submetida a um ruído branco. Assim, podemos expressar:

$$
p_{e q}(x, v)=\sqrt{\frac{k \gamma}{\pi D}} \exp \left[-\frac{k \gamma}{D} x^{2}\right] \sqrt{\frac{m \gamma}{\pi D}} \exp \left[-\frac{m \gamma}{D} v^{2}\right]
$$

\subsection{5}

\section{Resumo das estapas do método}

Por fim, podemos resumir a técnica para a obtenção da distribuição de probabilidade estacionária nos seguintes passos:

- calcular a transformada de Laplace da equação de Langevin e obter $\tilde{x}(s)$ e $\tilde{v}(s)$

- expressar a transformada de Laplace da distribuição da Eq. (3-9); 
- eliminar os termos nulos quando $z \rightarrow 0 \Leftrightarrow t \rightarrow \infty$;

- calcular as integrais dos termos remanescentes e obter a distribuição de equilíbrio.

Para o desenvolvimento do nosso trabalho no próximo capítulo, o cálculo da distribuição de probabilidade para a posição e velocidade é útil, em especial, para obter a distribuição para as condições iniciais. Na próxima seção, vamos exemplificar o uso da IJ para calibrar a função geradora de probabilidade do trabalho de um modelo de sistema microscópico diferente do descrito nesta seção.

\section{2}

\section{Função geradora de probabilidade para o trabalho de um sistema micros- cópico fora do equilíbrio}

O modelo estudado consiste de uma partícula Browniana de massa $m$, sob a ação de um potencial harmônico $k$, em contato com um reservatório térmico à temperatura $T$ e submetida a um coeficiente de fricção $\gamma$. De forma semelhante ao modelo ilustrado na Figura 2.1, uma extremidade de uma mola externa $\left(k^{\prime}\right)$ é fixada à partícula, e a outra extremidade é empurrada a uma taxa definida pelo protocolo $L(t)$, movendo-se ao redor de $x_{\text {mola }}=L(t)$. A interação entre o reservatório e a partícula é representada pelo ruído $\eta(t)$. O trabalho externo realizado sobre o sistema é o produto da força aplicada à extremidade móvel da mola $k^{\prime}$ pelo seu deslocamento $d L$.

A equação de Langevin que descreve o modelo é a seguinte:

$$
m \dot{v}(t)=-\gamma v(t)-k x(t)-k^{\prime}(x(t)-L(t))+\eta(t),
$$

na qual o protocolo do trabalho tem a forma

$$
L(t)=L_{0}\left(1-e^{-\frac{t}{\lambda}}\right) .
$$

A força estocástica $\eta(t)$ é um ruído branco Gaussiano. Portanto, o segundo cumulante é o único não nulo, e sua transformada de Laplace é expressa a seguir:

$$
\eta\left(s_{1}\right) \eta\left(s_{2}\right)=\frac{2 \gamma T}{s_{1}+s_{2}} .
$$

O processo pelo qual o sistema evolui percorre os seguintes passos:

- o processo começa no tempo $t=0$ com o sistema inicialmente em equilíbrio térmico com o reservatório à temperatura $T$;

- as condições iniciais da posição e velocidade são obtidas de acordo com a distribuição canônica à temperatura $T, \operatorname{com} L(t=0)=0$; 
- em seguida, a extremidade externa da mola é movida de acordo com o protocolo $L(t)$, de $t=0$ até $t=\tau$. A constante $\lambda$ tem unidade de tempo e, portanto, pode assumir qualquer valor positivo $(\lambda \rightarrow \infty$ corresponde a um processo reversível);

- o processo se encerra em $t=\tau$, podendo ser estendido para o limite $\tau \rightarrow \infty$ (equilíbrio termodinâmico).

A técnica para a obtenção da distribuição de probabilidade, da posição e velocidade, estudada na última seção, será utilizada de forma implícita para obter a função geradora do trabalho. Estas duas abordagens serão úteis para os procedimentos sobre o modelo proposto no quarto capítulo deste trabalho.

\subsection{1}

\section{Função geradora de probabilidade para o trabalho externo}

A função trabalho, que mede o trabalho realizado sobre o sistema no período $0<t<\tau$, é dada por:

$$
\begin{aligned}
W_{\tau} & =\int_{0}^{\tau} F_{e x t} d L=-k^{\prime} \int_{0}^{\tau} d t \frac{d L}{d t}(x(t)-L(t)) \\
& =\Delta U-k^{\prime} \int_{0}^{\tau} d t \frac{d L}{d t} x(t)
\end{aligned}
$$

Na qual, $F_{\text {ext }}=-k^{\prime}(x(t)-L(t))$ e $\Delta U=k^{\prime} \frac{L^{2}(\tau)}{2}$. A relação entre a taxa de variação do protocolo $L(t)$ e a posição $x(t)$ que dá origem ao trabalho irreversível.

Devido ao caráter linear da Eq. (3-28), a solução exata para $x(t)$ é a soma da solução homogênea $x_{h}(t)$, particular $x_{p}(t)$ e da fonte $x_{\eta}(t)$, isto é, $x(t)=x_{h}(t)+x_{p}(t) x_{\eta}(t)$. Logo, podemos expressar a função do trabalho na forma:

$$
\begin{aligned}
W_{\tau} & =\Delta U-k^{\prime} \int_{0}^{\tau} d t \frac{d L}{d t}\left(x_{h}(t)+x_{p}(t)+x_{\eta}(t)\right) . \\
& =\Delta U+\Delta W_{h}^{\tau}+\Delta W_{p}^{\tau}+\Delta W_{\eta}^{\tau}
\end{aligned}
$$

Utilizando o método de função de Green e as técnicas usuais de equações diferenciais, podemos encontrar as soluções para $x(t)[73,74]$. Não vamos nos estender nestes procedimentos para $x_{h}$ e $x_{p}$, visto que são soluções relativamente diretas, e expressaremos suas contribuição para o trabalho implicitamente:

$$
\Delta W_{h+p}^{\tau}=\Delta W_{h}^{\tau}+\Delta W_{p}^{\tau}=C_{1}^{\tau} x_{0}+C_{2}^{\tau} v_{0}+\Delta W_{p}^{\tau},
$$


na qual salientamos a relação de $\Delta W_{h}^{\tau}$ com as condições iniciais da posição e velocidade, $x_{0}$ e $v_{0}$ respectivamente.

A fim de construirmos a função geradora de probabilidade para o trabalho externo, precisamos tomar o valor esperado sobre o ruído térmico (daqui até o fim do trabalho representado por $\langle\bullet\rangle$ ) e sobre as condições inicias (daqui até o fim do trabalho representado por $\bullet$ ). A expressão nesta forma facilitará para a calibragem pela IJ, como é demonstrado mais adiante. Assim, a função geradora toma a forma:

$$
\begin{aligned}
\mathfrak{F}(u) & =\sum_{n=0}^{\infty} \frac{(-i u)^{n}}{n !} \overline{\left\langle W_{\tau}^{n}\right\rangle}=\sum_{n=0}^{\infty} \frac{(-i u)^{n}}{n !} \overline{\left\langle\left(\Delta U+\Delta W_{h}^{\tau}+\Delta W_{p}^{\tau}+\Delta W_{\eta}^{\tau}\right)^{n}\right\rangle} \\
& =\exp \left[-i u\left(\Delta U+W_{p}^{\tau}\right)\right] \sum_{n=0}^{\infty} \frac{(-i u)^{n}}{n !} \overline{\left\langle\left(\Delta W_{h}^{\tau}\right)^{n}\right\rangle} \sum_{n=0}^{\infty} \frac{(-i u)^{n}}{n !} \overline{\left\langle\left(\Delta W_{\eta}^{\tau}\right)^{n}\right\rangle}
\end{aligned}
$$

A solução para $x_{\eta}(t)$ é obtida pela contribuição da fonte na solução por função de Green, a saber [75, 65]:

$$
x_{\eta}(t)=\int_{0}^{t} d t^{\prime} \frac{2 \exp \left[-\theta\left(t-t^{\prime}\right) / 2\right]}{m \sqrt{4 \omega^{2}-\theta^{2}}} \operatorname{sen}\left(\frac{\sqrt{4 \omega^{2}-\theta^{2}}\left(t-t^{\prime}\right)}{2}\right) \eta\left(t^{\prime}\right),
$$

na qual $\theta=\gamma / m$ e $\omega^{2}=\frac{k+k^{\prime}}{m}$. Assim, a contribuição do termo do ruído para o trabalho realizado sobre o sistema é expresso da seguinte forma:

$$
\begin{aligned}
W_{\eta}^{\tau} & =-k^{\prime} \int_{0}^{\tau} d t \frac{d L}{d t} x_{\eta}(t) \\
& =-k^{\prime} \int_{0}^{\tau} d t \frac{L_{0}}{\lambda} e^{-t / \lambda} \int_{0}^{t} d t^{\prime} \frac{2 \exp \left[-\theta\left(t-t^{\prime}\right) / 2\right]}{m \sqrt{4 \omega^{2}-\theta^{2}}} \operatorname{sen}\left(\frac{\sqrt{4 \omega^{2}-\theta^{2}}\left(t-t^{\prime}\right)}{2}\right) \eta\left(t^{\prime}\right)
\end{aligned}
$$

Como já feito na última seção, vamos expressar o termo do ruído utilizando a transformada de Laplace como elemento central, de modo que:

$$
\begin{aligned}
\eta(t) & =\int_{0}^{\infty} \delta\left(t-t_{1}\right) \eta\left(t_{1}\right) d t_{1}=\lim _{\alpha \rightarrow 0^{+}} \int_{0}^{\infty} \int_{-\infty}^{\infty} \frac{d q_{1}}{2 \pi} e^{\left(i q_{1}+\alpha\right)\left(t-t_{1}\right)} \eta\left(t_{1}\right) d t_{1} \\
& =\lim _{\alpha \rightarrow 0^{+}} \int_{-\infty}^{\infty} \frac{d q_{1}}{2 \pi} e^{\left(i q_{1}+\alpha\right) t} \tilde{\eta}\left(i q_{1}+\alpha\right)
\end{aligned}
$$

Substituindo a Eq. (3-37) na Eq. (3-36), obtemos: 


$$
\begin{aligned}
W_{\eta}^{\tau} & =-\frac{2 L_{0} k^{\prime}}{m \lambda} \lim _{\alpha \rightarrow 0^{+}} \int_{-\infty}^{\infty} \frac{d q_{1}}{2 \pi} \tilde{\eta}\left(i q_{1}+\alpha\right) \times \\
& \times \int_{0}^{\tau} d t \int_{0}^{t} d t^{\prime} \frac{e^{\left(i q_{1}+\alpha\right) t^{\prime}-t / \lambda-\theta\left(t-t^{\prime}\right) / 2}}{\sqrt{4 \omega^{2}-\theta^{2}}} \operatorname{sen}\left(\frac{\sqrt{4 \omega^{2}-\theta^{2}}\left(t-t^{\prime}\right)}{2}\right) .
\end{aligned}
$$

O argumento da última linha da Eq. (3-38) é integrável sobre $t$ e $t^{\prime}$ e pode ser expresso como a soma a seguir:

$W_{\eta}^{\tau}=-\lim _{\alpha \rightarrow 0^{+}} \int_{-\infty}^{\infty} \frac{d q_{1}}{2 \pi} \tilde{\eta}\left(i q_{1}+\alpha\right)\left[W_{\eta 1}^{\tau}\left(i q_{1}+\alpha\right)+W_{\eta 2}^{\tau}\left(i q_{1}+\alpha\right) W_{\eta 3}^{\tau}\left(i q_{1}+\alpha\right) W_{\eta 4}^{\tau}\left(i q_{1}+\alpha\right)\right]$.

Na qual,

$$
\begin{aligned}
W_{\eta 1}^{\tau}(s) & =\frac{k^{\prime} L_{0}}{m\left(\theta s+s^{2}+\omega^{2}\right)(s \lambda-1)} e^{\tau(s \lambda-1) / \lambda}, \\
W_{\eta 2}^{\tau}(s) & =-\frac{k^{\prime} L_{0} \lambda^{2}}{m(s \lambda-1)\left(\theta \lambda+1+\omega^{2} \lambda^{2}\right)}, \\
W_{\eta 3}^{\tau}(s) & =-\frac{\left(-\theta \lambda s-\theta^{2} \lambda+2 \lambda \omega^{2}-2 s-\theta\right) k^{\prime} L_{0}}{m \sqrt{4 \omega^{2}-\theta^{2}}\left(\theta s+s^{2}+\omega^{2}\right)\left(\theta \lambda+1+\omega^{2} \lambda^{2}\right)} e^{-\tau(2+\theta \lambda) / 2 \lambda} \operatorname{Sen}\left(\frac{\tau \sqrt{4 \omega^{2}-\theta^{2}}}{2}\right), \\
W_{\eta 4}^{\tau}(s) & =\frac{(s \lambda+\theta \lambda+1) k^{\prime} L_{0}}{m\left(\theta s+s^{2}+\omega^{2}\right)\left(\theta \lambda+1+\omega^{2} \lambda^{2}\right)} e^{-\tau(2+\theta \lambda) / 2 \lambda} \operatorname{Cos}\left(\frac{\tau \sqrt{4 \omega^{2}-\theta^{2}}}{2}\right) .
\end{aligned}
$$

Para calcularmos os momentos $\left\langle\left(\Delta W_{\eta}^{\tau}\right)^{n}\right\rangle$ do trabalho, devemos nos atentar às características da distribuição Gaussiana $\eta(t)$. Daí se conclui que todos os momentos ímpares serão nulos, e os momentos pares remanescentes são produtos do tipo $\left\langle W_{\eta}^{\tau} W_{\eta}^{\tau}\right\rangle$. A integral típica a ser calculada é:

$$
\begin{aligned}
I_{i j} & =\lim _{\alpha \rightarrow 0^{+}} \int_{-\infty}^{\infty} \frac{d q_{1}}{2 \pi} \int_{-\infty}^{\infty} \frac{d q_{2}}{2 \pi}\left\langle\tilde{\eta}\left(i q_{1}+\alpha\right) \tilde{\eta}\left(i q_{2}+\alpha\right)\right\rangle W_{\eta i}^{\tau}\left(i q_{1}+\alpha\right) W_{\eta j}^{\tau}\left(i q_{2}+\alpha\right) \\
& =\lim _{\alpha \rightarrow 0^{+}} \int_{-\infty}^{\infty} \frac{d q_{1}}{2 \pi} \int_{-\infty}^{\infty} \frac{d q_{2}}{2 \pi} \frac{2 \gamma T}{i q_{1}+i q_{2}+2 \epsilon} W_{\eta i}^{\tau}\left(i q_{1}+\alpha\right) W_{\eta j}^{\tau}\left(i q_{2}+\alpha\right) . \quad(3-44)
\end{aligned}
$$

A susbstituição da última linha é devido à expressão do segundo cumulante de $\eta(t)$ dado na Eq. (3-30).

Das dez combinações sem repetições possíveis para a integral $I_{i j}$, apenas os termos $I_{11}, I_{12}$ e $I_{22}$ não são nulos. Assim, o valor esperado dos momentos 
do trabalho $\left\langle\left(\Delta W_{\eta}^{\tau}\right)^{n}\right\rangle$ são as combinações 2 a 2 que podem ser feitas com a soma das integrais $I_{i j}$, como segue:

na qual $n=2 m$.

$$
\left\langle\left(\Delta W_{\eta}^{\tau}\right)^{2 m}\right\rangle=\frac{(2 m) !}{m ! 2^{m}}\left(I_{11}+2 I_{12}+I_{22}\right)^{m},
$$

Assim, por notadamente $\Delta W_{\eta}^{\tau}$ não depender das condições iniciais, podemos escrever:

$$
\sum_{n=0}^{\infty} \frac{(-i u)^{n}}{n !} \overline{\left\langle\left(\Delta W_{\eta}^{\tau}\right)^{n}\right\rangle}=\exp \left[-u^{2} \frac{I_{11}+2 I_{12}+I_{22}}{2}\right] .
$$

Para calcularmos o termo $\overline{\left\langle\left(\Delta W_{h}^{\tau}\right)^{n}\right\rangle}=\overline{\left\langle\left(C_{1}^{\tau} x_{0}+C_{2}^{\tau} v_{0}\right)^{n}\right\rangle}$, devemos considerar as caracterísiticas da distribuição canônica das condições iniciais. Como a função de partição canônica no equilíbrio é dada por

$$
Z=\int_{-\infty}^{\infty} \frac{d p d x}{\hbar} e^{-\beta H(x, p)},
$$

na qual $\beta H(x, p)=\frac{1}{2 T}\left(\frac{(m v)^{2}}{m}+k x^{2}+k^{\prime}(x-L)^{2}\right)$, a distribuição de probabilidade para as condições iniciais também possui características Gaussianas. Logo, os únicos termos relevantes são:

$$
\overline{\left\langle\left(\Delta W_{h}^{\tau}\right)^{n}\right\rangle}=\frac{(2 m) !}{m ! 2^{m}}\left[\left(C_{1}^{\tau}\right)^{2} \overline{\left(x_{0}\right)^{2}}+\left(C_{2}^{\tau}\right)^{2} \overline{\left(v_{0}\right)^{2}}\right],
$$

na qual $\overline{\left(x_{0}\right)^{2}}=\frac{T}{m \omega^{2}}, \overline{\left(v_{0}\right)^{2}}=\frac{T}{m}$ e $n=2 m$. Por notadamente $\Delta W_{h}^{\tau}$ não depender do ruído $\eta(t)$ e os termos cruzados não contribuirem no equilíbrio, podemos escrever:

$$
\sum_{n=0}^{\infty} \frac{(-i u)^{n}}{n !} \overline{\left\langle\left(\Delta W_{h}^{\tau}\right)^{n}\right\rangle}=\exp \left[-u^{2} \frac{\left(C_{1}^{\tau}\right)^{2} \overline{\left(x_{0}\right)^{2}}+\left(C_{2}^{\tau}\right)^{2} \overline{\left(v_{0}\right)^{2}}}{2}\right]
$$

Finalmente, substituindo os resultados das Eqs. (3-46) e (3-49) na Eq.(3$34)$, obtemos a função geradora de probabilidade para o trabalho realizado sobre o sistema:

$$
\mathfrak{F}(u)=\exp \left[-i u\left(\Delta U+W_{p}^{\tau}\right)-u^{2} \frac{I_{11}+2 I_{12}+I_{22}}{2}-u^{2} \frac{\left(C_{1}^{\tau}\right)^{2} \overline{\left(x_{0}\right)^{2}}+\left(C_{2}^{\tau}\right)^{2} \overline{\left(v_{0}\right)^{2}}}{2}\right] .
$$

Os termos omitidos não interferem na descrição do método utilizado e podem ser verificados em [65]. 


\subsection{2}

\section{Igualdade de Jarzynski}

Tomando a definição da energia livre de Helmholtz da Eq. (2-12) e substituindo a função de partição da Eq. (3-47), a energia livre do sistema é obtida e expressa na forma:

$$
F=\left(\frac{k k^{\prime}}{k+k^{\prime}}\right) \frac{L^{2}}{2}-T \ln \left(\frac{2 \pi T}{\bar{h}} \sqrt{\frac{m}{k+k^{\prime}}}\right),
$$

na qual $L$ é a extensão total da mola e, via teorema da equipartição, $T$ é a soma da contribuição da energia cinética e energia potencial elástica no equilíbrio [26].

Após algum algebrismo, substituindo $u=-i / T$ na Eq. (3-50), é verificada a IJ para o sistema, de forma que:

$$
\mathfrak{F}\left(-\frac{i}{T}\right)=\left\langle\exp \left(-\frac{W_{\tau}}{T}\right)\right\rangle=\exp \left(-\frac{\Delta F}{T}\right),
$$

na qual $\Delta F=\left(\frac{k k^{\prime}}{k+k^{\prime}}\right) \frac{L^{2}(\tau)}{2}$ e $L(t=0)=0$.

Mantendo $T$ constante, o trabalho reversível $W_{r}$ e a energia livre $\Delta F$ possuem valores idênticos. Logo,

$$
W_{r}=\Delta F=\left(\frac{k k^{\prime}}{k+k^{\prime}}\right) \frac{L^{2}}{2}
$$

Por fim, podemos expressar o trabalho dissipativo $W_{d}=W_{\tau}-W_{r}$ como a integral das flutuações ao redor da posição instantânea de equilíbrio $x^{e q}=\frac{k^{\prime}}{k+k^{\prime}} L(t)$, como segue:

$$
W_{d}=-k^{\prime} \int_{0}^{\tau} d t \frac{d L}{d t}\left(x(t)-\frac{k^{\prime}}{k+k^{\prime}} L(t)\right) .
$$

As técnicas apresentadas neste capítulo já estão bem consolidadas na literatura. São procedimentos muito úteis para a obtenção da solução de alguns tipos de equações diferenciais estocásticas. Em especial, essas técnicas se mostraram adequadas para o desenvolvimento do trabalho original desta tese, que pode ser conferido no próximo capítulo. 


\section{4}

\section{Modelo não markoviano linear e não linear}

No primeiro capítulo deste trabalho, descrevemos como podemos obter informações de sistemas microscópicos em não-equilíbrio utilizando a igualdade de Jarzynski [8]:

$$
\langle\exp (-\beta W)\rangle=\exp (-\beta \Delta F)
$$

A validade dessa relação tem sido amplamente verificada para sistemas determinísticos e markovianos [18, 76, 61]. Propomos neste capítulo um modelo não markoviano linear estocástico, cuja calibração é feita pela IJ. Além disso, calculamos a produção de entropia fora do equilíbrio para o sistema modelado. Também, apresentamos os resultados da simulação para o modelo não-linear deste sistema. Por último, derivamos um resultado interessante para o trabalho dissipado a partir da IJ.

\section{1 \\ O modelo não linear}

O movimento browniano é um dos mais fundamentais exemplos de propriedadades de transporte que surgem de flutuações próximas do equilíbrio termodinâmico. Contudo, a teoria do movimento browniano de Einstein é um processo tipicamente markoviano. A escala de tempo de dissipação da energia de uma colisão da partícula browniana com as partículas do ambiente é muito maior que o tempo entre duas colisões. Essa característica é que confere ao movimento browniano o perfil randômico, pois, em uma escala de tempo muito maior que a escala de tempo de dissipação da energia das colisões, o movimento da partícula browniana aparenta ser markoviano [77]. Essa abordagem é limitada à análise de somente uma fração dos problemas de flutuações que enfrentamos. Em outras palavras, frequentemente, a partícula, cujo comportamento estamos descrevendo, possui massa similar à massa das partículas do ambiente. Esta propriedade induz uma dinâmica não markoviana ao sistema.

Neste capítulo, propomos um modelo que contemple alguns casos analiticamente solúveis. Apesar de ser similar na abordagem feita em [65], ele contém 
características que induzem o comportamento não markoviano do sistema. Tais características serão melhor definidas mais adiante. A equação diferencial de Langevin, a qual descreve a evolução temporal do nosso sistema, é definida na forma:

$$
\begin{aligned}
m \dot{v}(t) & =-\Upsilon(t)-k_{1} x(t)-k_{2}[x(t)-L(t)]-k_{3} x^{3}(t)+\xi(t) \\
x(t) & =x_{0}+\int_{0}^{t} v\left(t^{\prime}\right) d t^{\prime} \\
L(t) & =L_{0}\left(1-e^{-\frac{t}{\lambda}}\right) \\
\Upsilon(t) & =\int_{0}^{t} d t^{\prime} \phi\left(t-t^{\prime}\right) v\left(t^{\prime}\right) .
\end{aligned}
$$

Na qual $\xi(t)$ é um ruído Gaussiano caracterizado por

$$
\begin{aligned}
\langle\xi(t)\rangle & =0 \\
\left\langle\xi(t) \xi\left(t^{\prime}\right)\right\rangle & =\frac{\gamma T}{\tau} e^{-\frac{\left|t-t^{\prime}\right|}{\tau}}, \\
\phi\left(t-t^{\prime}\right) & =\frac{\left\langle\xi(t) \xi\left(t^{\prime}\right)\right\rangle}{T} .
\end{aligned}
$$

A Eq. (4-2) pode ser vista como uma Equação Generalizada de Langevin [78], na qual a partícula está submetida a um potencial de acordo com a expressão:

$$
U(x)=\frac{k_{1}}{2} x^{2}+\frac{k_{2}}{2}[x-L]^{2}+\frac{k_{3}}{4} x^{4} .
$$

Diferentemente do modelo contido em [65], o último termo é um potencial generalizado, no sentido de que já não se trata mais de um potencial harmônico, substituído neste trabalho por um potencial não harmônico. O segundo termo $\left(k_{2}\right)$ pode ser visto como uma mola externa fixada a uma extremidade da partícula, que se move em torno do ponto de equilíbrio, enquanto a outra extremidade é puxada com uma taxa definida pelo protocolo $L(t)$. Já o primeiro termo é definido como o potencial intrínseco da partícula que está sob a análise.

O ruído gaussiano $\xi(t)$ e a dissipação $\Upsilon\left(t, t^{\prime}\right)$ são as componentes estocásticas associadas ao efeito das colisões da partícula com a vizinhança [77]. Estas componentes que induzem a randomicidade no nosso modelo. A função de correlação temporal de $\xi(t)$ é aproximada pela Eq. (4-7). O efeito das colisões, entre o banho térmico e a partícula browniana, se manifestam em $\xi(t)$ e $\phi\left(t-t^{\prime}\right)$, causando uma dinâmica intrinsicamente não-Markoviana. As constantes $\gamma, \tau$ e $T$ são o coeficiente de fricção, uma constante com dimensão de tempo, que pode ser escolhida sem perda de generalidade, e a temperatura do banho térmico (ambiente), respectivamente.

Por conveniência, vamos expressar o termo espacial da força cúbica 
$k_{3} x^{3}(t)$ da seguinte forma:

$$
\begin{aligned}
k_{3} x^{3}(t) & =k_{3} \lim _{\alpha \rightarrow 0^{+}} \int_{-\infty}^{\infty} \int_{-\infty}^{\infty} \int_{-\infty}^{\infty} \frac{d q_{1}}{2 \pi} \frac{d q_{2}}{2 \pi} \frac{d q_{3}}{2 \pi} e^{\left(i q_{1}+i q_{2}+i q_{3}+3 \alpha\right) t} \times \\
& \times \tilde{x}\left(i q_{1}+\alpha\right) \tilde{x}\left(i q_{2}+\alpha\right) \tilde{x}\left(i q_{3}+\alpha\right) .
\end{aligned}
$$

Na qual $q_{1}, q_{2}, q_{3} \in \mathbb{R}^{*}+$. Esta forma de representar o termo cúbico é mais adequada ao modo que vamos atacar o problema. Isto ficará mais evidente quando calcularmos a solução da equação de Langevin. A dedução completa dessa expressão pode ser verificada no apêndice C.1.

Aplicando a transformada de Laplace à equação e evidenciando $\tilde{x}(s)$, encontramos a solução geral da Eq. (4-2) a seguir:

$$
\begin{aligned}
\tilde{x}(s) & =\frac{x_{0}\left(\gamma+m s^{2} \tau+m s\right)+v_{0}(m s \tau+m)}{R(s)}+ \\
+\quad & \frac{\xi(s)(s \tau+1)}{R(s)}+\frac{k_{2} L_{0}(s \tau+1)}{s(\lambda s+1) R(s)}- \\
-\quad & k_{3} \frac{\lim _{\alpha \rightarrow 0^{+}} \int_{-\infty}^{\infty} \int_{-\infty}^{\infty} \int_{-\infty}^{\infty} \frac{d q_{1}}{2 \pi} \frac{d q_{2}}{2 \pi} \frac{d q_{3}}{2 \pi} \frac{\tilde{x}\left(i q_{1}+\alpha\right) \tilde{x}\left(i q_{2}+\alpha\right) \tilde{x}\left(i q_{3}+\alpha\right)}{s-\left(i q_{1}+i q_{2}+i q_{3}+3 \alpha\right)}(s \tau+1)}{R(s)} .
\end{aligned}
$$

$\mathrm{Na}$ qual

$$
R(s)=(s \tau+1)\left(k_{1}+k_{2}+m s^{2}\right)+\gamma s .
$$

As raízes $\kappa_{1}, \kappa_{2}$ e $\kappa_{3}$ de $R(s)$ existem e podem ser encontradas de forma analítica.

Ainda, podemos expressar a energia do sistema através da seguinte Hamiltoniana:

$$
H=\frac{m v^{2}}{2}+\frac{k_{1} x^{2}}{2}+\frac{k_{2}}{2}(x-L)^{2}+\frac{k_{3} x^{4}}{4} .
$$

O último procedimento desta seção será derivar a expressão da primeira lei da termodinâmica (Eq. (2-3)) para a Eq. (4-2). Para isto, vamos multiplicar esta última por $v(t)$, e deixar em evidência os termos não relacionados com o trabalho externo e o ruído térmico, resultando na expressão:

$$
\left(m \dot{v}(t)+k_{1} x(t)+k_{3} x^{3}(t)\right) v(t)=-\Upsilon(t) v(t)+\xi(t) v(t)-k_{2}[x(t)-L(t)] v(t)
$$




$$
\begin{gathered}
\frac{d}{d t}\left(\frac{m v^{2}(t)}{2}+\frac{k_{1} x^{2}(t)}{2}+\frac{k_{3} x^{4}(t)}{4}\right)=\frac{d Q(t)}{d t}-\frac{d}{d t}\left(\frac{k_{2}}{2}[x(t)-L(t)]^{2}\right)-k_{2}[x(t)-L(t)] \frac{d L(t)}{d t} \\
\frac{d}{d t}\left(\frac{m v^{2}(t)}{2}+\frac{k_{1} x^{2}(t)}{2}+\frac{k_{3} x^{4}(t)}{4}+\frac{k_{2}}{2}[x(t)-L(t)]^{2}\right)=\frac{d Q(t)}{d t}-k_{2}[x(t)-L(t)] \frac{d L(t)}{d t} \\
\frac{d U(t)}{d t}=\frac{d Q(t)}{d t}+\frac{d W(t)}{d t} .
\end{gathered}
$$

Na qual

$$
\begin{gathered}
\frac{d U(t)}{d t}=\frac{d}{d t}\left(\frac{m v^{2}(t)}{2}+\frac{k_{1} x^{2}(t)}{2}+\frac{k_{3} x^{4}(t)}{4}+\frac{k_{2}}{2}[x(t)-L(t)]^{2}\right), \\
\frac{d Q(t)}{d t}=-\Upsilon(t) v(t)+\xi(t) v(t) \\
\frac{d W(t)}{d t}=-k_{2}[x(t)-L(t)] \frac{d L(t)}{d t}
\end{gathered}
$$

são o fluxo da energia interna, o fluxo do calor e o fluxo do trabalho para o sistema de interesse, respectivamente. Esta conclusão segue de forma similar à interpretação da Eq. (2-15) do primeiro capítulo: se não houvesse a ação do protocolo, teríamos somente a contribuição do termo do fluxo do calor na Eq. (4-14). O protocolo $L(t)$ representa uma maneira ordenada e controlada do sistema de interesse trocar energia, por meio do trabalho, com o sistema externo.

\subsection{1}

\section{Etapas do processo termodinâmico}

As etapas do processo termodinâmico são as mesmas utilizadas para a obtenção da função geradora do trabalho, no capítulo anterior. Vamos relembrá-las: partindo do equilíbrio termodinâmico, os seguintes passos do processo são seguidos:

- O processo começa em $t=0$, com $L=0$;

- O sistema está em equilíbrio com um reservatório à temperatura $T$, e suas condições iniciais $\left(x_{0}, v_{0}\right)$ são obtidas através da distribuição de Boltzmann-Gibbs $(L=0)$;

- No tempo $t=0^{+}$uma força externa é aplicada, causando um deslocamento descrito pelo protocolo $L(t)=L_{0}\left(1-e^{-t / \lambda}\right)$, e realiza um trabalho $W$ sobre o sistema até o instante $t=\tau$. (O formato do protocolo $L(t)$ pode ser escolhido sem perda de generalidade, de modo que é possível substituí-lo por formas mais complexas. A forma do ruído branco é 
recuperada quando $\tau \rightarrow 0$, e podemos também estabelecer a condição $\tau \rightarrow \infty)$

- O processo termodinâmico descrito nos ítens anteriores é repetido diversas vezes, medindo-se o trabalho $W$ para cada iteração, e a média $\langle\exp (-\beta W)\rangle$ é calculada sobre o ensemble;

- A energia livre é calculada para os pontos de equilíbrio, nos quais $\Delta F=F(t=\tau)-F(t=0)$.

\subsection{2}

\section{Cumulantes do trabalho $W$}

O trabalho externo realizado pelo sistema é dado por

$$
\begin{aligned}
& W_{\theta}=-k_{2} \int_{0}^{\theta} d t \frac{d L}{d t}(x(t)-L(t)) \\
& W_{\theta}=-k_{2}\left(\int_{0}^{\theta} d t \frac{d L}{d t} x(t)-\int_{0}^{\theta} d t \frac{d L}{d t} L(t)\right) \\
& W_{\theta}=-k_{2} \int_{0}^{\theta} d t \frac{d L}{d t} x(t)+k_{2} \frac{L_{\theta}^{2}}{2} \\
& W_{\theta}=I_{\theta}+\Delta U
\end{aligned}
$$

no qual

$$
\begin{aligned}
I_{\theta} & =-k_{2} \int_{0}^{\theta} d t \frac{d L}{d t} x(t), \\
\Delta U & =k_{2} \frac{L_{\theta}^{2}}{2} .
\end{aligned}
$$

O trabalho é o produto da força externa dependente do tempo, aplicada à extremidade móvel da mola $k_{2}$, pelo deslocamento espacial $d L$ [65]. Por meio do protocolo $L(t)$ é possível ajustar a troca de trabalho $W$ e entropia $S$ com o ambiente. O protocolo utilizado é equivalente a outros já utilizados em abordagens similares [79].

Para obtermos os cumulantes do trabalho $W_{\theta}$, vamos reescrever a integral $I_{\theta}$ deste modo: 


$$
\begin{aligned}
I_{\theta} & =-k_{2} \int_{0}^{\theta} d t \frac{d L}{d t} x(t) \\
I_{\theta} & =-\frac{k_{2} L_{0}}{\lambda} \int_{0}^{\theta} d t \quad e^{-\frac{t}{\lambda}} \int_{0}^{\infty} d t_{1} \quad \delta\left(t-t_{1}\right) x\left(t_{1}\right) \\
I_{\theta} & =-\frac{k_{2} L_{0}}{2 \pi \lambda} \int_{0}^{\theta} d t \quad e^{-\frac{t}{\lambda}} \int_{0}^{\infty} d t_{1} \int_{-\infty}^{\infty} d q_{1} \quad e^{\left(i q_{1}+\epsilon\right)\left(t-t_{1}\right)} x\left(t_{1}\right) \\
I_{\theta} & =-\frac{k_{2} L_{0}}{2 \pi \lambda} \int_{0}^{\theta} d t \quad e^{-\frac{t}{\lambda}} \int_{-\infty}^{\infty} d q_{1} \quad e^{\left(i q_{1}+\epsilon\right) t} \tilde{x}\left(i q_{1}+\epsilon\right) \\
I_{\theta} & =\frac{k_{2} L_{0}}{2 \pi \lambda} \int_{-\infty}^{\infty} d q_{1} \frac{e^{-\theta\left(\frac{1}{\lambda}-i q_{1}-\epsilon\right)}-1}{\frac{1}{\lambda}-i q_{1}-\epsilon} \tilde{x}\left(i q_{1}+\epsilon\right) .
\end{aligned}
$$

Assim, utilizando a Eq. (4-22), podemos facilmente escrever a função geradora de probabilidade (FGP) e a função geradora de cumulantes (FGC) do trabalho $W$, que são expressas respectivamente na forma

$$
G(u) \equiv\left\langle\overline{\exp \left[-i u W_{\theta}\right]}\right\rangle=\sum_{n=1}^{\infty} \frac{(-i u)^{n}}{n !}\left\langle\overline{W_{\theta}^{n}}\right\rangle=e^{-i u \Delta U} \sum_{n=0}^{\infty} \frac{(-i u)^{n}}{n !}\left\langle\overline{I_{\theta}^{n}}\right\rangle
$$

$$
\ln G(u)=\ln \left\langle\overline{\exp \left[-i u W_{\theta}\right]}\right\rangle=-i u \Delta U+\sum_{n=1}^{\infty} \frac{(-i u)^{n}}{n !}\left\langle\overline{I_{\theta}^{n}}\right\rangle
$$

No qual o valor esperado $\langle\bullet\rangle$ é tomado sobre o ruído térmico $\xi(t)$, o qual corresponde a todos os caminhos fora do equilíbrio possíveis para a partícula Browniana. Já a média • é tomada sobre a função de distribuição de probabilidade das condições iniciais $\left(x_{0}\right.$ e $\left.v_{0}\right)$.

\section{2}

\section{Caso linear $\left(k_{3}=0\right)$}

Nesta seção vamos considerar o caso linear, isto é, quando $k_{3}=0$. Primeiramente, vamos obter a função de distribuição de probabilidade das condições iniciais. A distribuição de equilíbrio para a posição $x$ é dada por

$$
\begin{aligned}
p(x, t) & =\langle\delta(x-x(t))\rangle_{\xi} \\
& =\lim _{\epsilon \rightarrow 0} \sum_{m=0}^{\infty} \frac{1}{m !} \int_{-\infty}^{\infty} \frac{d Q}{2 \pi}(-i Q)^{m} e^{i Q x} \int_{-\infty}^{\infty} \prod_{i=1}^{m} \frac{d q_{i}}{2 \pi} e^{\sum_{j=1}^{m}\left(i q_{j}+\epsilon\right) t}\left\langle\prod_{i=1}^{m} \tilde{x}\left(i q_{i}+\epsilon\right)\right\rangle_{\xi}
\end{aligned}
$$

No qual $\langle\bullet\rangle_{\xi}$ é o valor esperado tomado sobre o ruído térmico. Para obtermos a solução estacionária, basta que tomemos $t \rightarrow \infty$ ou a média temporal sobre 
$4-25$.

Para o caso linear, podemos expressar $\tilde{x}(s)$ na forma

$$
\begin{aligned}
\tilde{x}(s) & =\frac{x_{0}\left(\gamma+m s^{2} \tau+m s\right)+v_{0}(m s \tau+m)}{R(s)}+\frac{\xi(s)(s \tau+1)}{R(s)}+ \\
& +\frac{k_{2} L_{0}(s \tau+1)}{s(\lambda s+1) R(s)}
\end{aligned}
$$

Substituindo a Eq. (4-26) na Eq. (4-25), e aplicando o limite $t \rightarrow \infty$, obtemos a seguinte distribuição:

$$
\begin{aligned}
p(x) & =\lim _{t \rightarrow \infty} p(x, t) \\
& =\lim _{t \rightarrow \infty} \lim _{\epsilon \rightarrow 0} \sum_{m=0}^{\infty} \frac{1}{m !} \int_{-\infty}^{\infty} \frac{d Q}{2 \pi}(-i Q)^{m} e^{i Q x} \int_{-\infty}^{\infty} \prod_{i=1}^{m} \frac{d q_{i}}{2 \pi} e^{\sum_{j=1}^{m}\left(i q_{j}+\epsilon\right) t} \times \\
& \times \frac{1}{\prod_{i=1}^{m} R\left(i q_{i}+\epsilon\right)}\left\langle\prod _ { i = 1 } ^ { m } \left( x_{0}\left(\gamma+m\left(i q_{i}+\epsilon\right)^{2} \tau+m\left(i q_{i}+\epsilon\right)\right)+\right.\right. \\
& +v_{0}\left(m\left(i q_{i}+\epsilon\right) \tau+m\right)+\left(\left(i q_{i}+\epsilon\right) \tau+1\right) \tilde{\xi}\left(i q_{i}+\epsilon\right)+ \\
& \left.\left.+\frac{k_{2} L_{0}\left(\left(i q_{i}+\epsilon\right) \tau+1\right)}{\left(i q_{i}+\epsilon\right)\left(\lambda\left(i q_{i}+\epsilon\right)+1\right)}\right)\right\rangle_{\xi}
\end{aligned}
$$

Na qual $p(\star)$ é a função de distribuição estacionária de probabilidade para o caso linear $\left(k_{3}=0\right)$.

Devido à natureza gaussiana do ruído térmico, o valor esperado dos $m$ termos que não desaparecem são aqueles que atendem à condição $m=2 n$. Então, podemos reescrever o argumento da média sobre o ruído na forma

$$
\begin{array}{cl}
\prod_{i=1}^{n} & {\left[\left(x_{0}\left(\gamma+m\left(i q_{i}+\epsilon\right)^{2} \tau+m\left(i q_{i}+\epsilon\right)\right)+\right.\right.} \\
+ & \left.v_{0}\left(m\left(i q_{i}+\epsilon\right) \tau+m\right)+\frac{k_{2} L_{0}\left(\left(i q_{i}+\epsilon\right) \tau+1\right)}{\left(i q_{i}+\epsilon\right)\left(\lambda\left(i q_{i}+\epsilon\right)+1\right)}\right) \times \\
\times & \left(x_{0}\left(\gamma+m\left(i q_{i+1}+\epsilon\right)^{2} \tau+m\left(i q_{i+1}+\epsilon\right)\right)+\right. \\
+ & \left.v_{0}\left(m\left(i q_{i+1}+\epsilon\right) \tau+m\right)+\frac{k_{2} L_{0}\left(\left(i q_{i+1}+\epsilon\right) \tau+1\right)}{\left(i q_{i+1}+\epsilon\right)\left(\lambda\left(i q_{i+1}+\epsilon\right)+1\right)}\right)+ \\
+ & \left.\left(\left(i q_{i}+\epsilon\right) \tau+1\right)\left(\left(i q_{i+1}+\epsilon\right) \tau+1\right) \tilde{\xi}\left(i q_{i}+\epsilon\right) \tilde{\xi}\left(i q_{i+1}+\epsilon\right)\right] .
\end{array}
$$

Por escolhermos que o argumento do produtório seja um produto par a par, o índice $i$ só admite valores ímpares.

Os termos em 4-28 são associados 2 a 2. Desse modo, é necessário fazer a contagem dos diversos acoplamentos possíveis para o argumento. Para este 
tipo de combinação o valor que deve ser utilizado é $\frac{(2 n) !}{n ! 2^{n}}$. Portanto, a integral típica que deve ser calculada será

$$
\begin{array}{ll}
I_{\xi \xi} & =\lim _{t \rightarrow \infty} \lim _{\epsilon \rightarrow 0} \int_{\infty}^{\infty} \frac{d q_{1}}{2 \pi} \frac{d q_{2}}{2 \pi} \frac{e^{i q_{1}+i q_{1}+2 \epsilon}}{R\left(i q_{1}+\epsilon\right) R\left(i q_{2}+\epsilon\right)} \times \\
\times & {\left[\left(x_{0}\left(\gamma+m\left(i q_{i}+\epsilon\right)^{2} \tau+m\left(i q_{i}+\epsilon\right)\right)+\right.\right.} \\
+ & \left.v_{0}\left(m\left(i q_{i}+\epsilon\right) \tau+m\right)+\frac{k_{2} L_{0}\left(\left(i q_{i}+\epsilon\right) \tau+1\right)}{\left(i q_{i}+\epsilon\right)\left(\lambda\left(i q_{i}+\epsilon\right)+1\right)}\right) \times \\
\times \quad & \left(x_{0}\left(\gamma+m\left(i q_{i+1}+\epsilon\right)^{2} \tau+m\left(i q_{i+1}+\epsilon\right)\right)+\right. \\
+ & \left.v_{0}\left(m\left(i q_{i+1}+\epsilon\right) \tau+m\right)+\frac{k_{2} L_{0}\left(\left(i q_{i+1}+\epsilon\right) \tau+1\right)}{\left(i q_{i+1}+\epsilon\right)\left(\lambda\left(i q_{i+1}+\epsilon\right)+1\right)}\right)+ \\
+ & \left.\left(\left(i q_{i}+\epsilon\right) \tau+1\right)\left(\left(i q_{i+1}+\epsilon\right) \tau+1\right) \tilde{\xi}\left(i q_{i}+\epsilon\right) \tilde{\xi}\left(i q_{i+1}+\epsilon\right)\right] .
\end{array}
$$

Após a realização da integral, considerando o segundo cumulante do ruído, a distribuição estacionária obtida para a posição da partícula Browniana será

$$
\begin{aligned}
p(x) & =\sum_{n=0}^{\infty} \frac{1}{(2 n) !} \int_{-\infty}^{\infty} \frac{d Q}{2 \pi}(-i Q)^{2 n} e^{i Q x} \frac{(2 n) !}{n ! 2^{n}} I_{\xi \xi}^{n} \\
& =\int_{-\infty}^{\infty} \frac{d Q}{2 \pi} e^{i Q x} \sum_{n=0}^{\infty} \frac{1}{n !}\left(-\frac{Q^{2}}{2} I_{\xi \xi}\right)^{n} \\
& =\int_{-\infty}^{\infty} \frac{d Q}{2 \pi} e^{i Q x-\frac{Q^{2}}{2} I_{\xi \xi}} \\
p(x) & =\frac{\sqrt{\left(k_{1}+k_{2}\right) m}}{2 \pi T} e^{-\frac{\left(k_{1}+k_{2}\right)}{4 T}\left(x-\frac{k_{2} L}{k_{1}+k_{2}}\right)^{2}} .
\end{aligned}
$$

Os cálculos da distribuição estacionária para a velocidade são similares e, de forma análoga ao resultado da Eq. (3-25) do capítulo anterior, os termos cruzados são nulos. Portanto, a distribuição para a posição e velocidade é o produto de ambas as distribuições isoladamente. Assim, obtemos

$$
p(x, v)=\frac{\sqrt{\left(k_{1}+k_{2}\right) m}}{2 \pi T} e^{-\frac{m v^{2}}{2 T}-\frac{\left(k_{1}+k_{2}\right)}{2 T}\left(x-\frac{k_{2} L}{k_{1}+k_{2}}\right)^{2}} .
$$

Apesar da atuação do termo de memória, a distribução de probabilidade, para a posição e velocidadde, é tipo Boltzmann-Gibbs no equilíbrio. Nas próximas duas seções, utilizaremos a função de distribuição da Eq. (4-31) para calcularmos o primeiro e o segundo cumulante do trabalho. 


\subsection{1}

\section{Primeiro cumulante $\left\langle\overline{I_{\theta}}\right\rangle$}

A fim de obtermos os cumulantes do trabalho $W$, precisamos calcular, primeiramente, o cumulante da posição para o caso linear. Para isso, é preciso encontrar a solução da seguinte integral:

$$
\begin{aligned}
& \left\langle\overline{I_{\theta}}\right\rangle=\left\langle\overline{\frac{k_{2} L_{0}}{2 \pi \lambda} \int_{-\infty}^{\infty} d q_{1} \frac{e^{-\theta\left(\frac{1}{\lambda}-i q_{1}-\epsilon\right)}-1}{\frac{1}{\lambda}-i q_{1}-\epsilon} \tilde{x}\left(i q_{1}+\epsilon\right)}\right\rangle \\
& \left\langle\overline{I_{\theta}}\right\rangle=\frac{k_{2} L_{0}}{2 \pi \lambda} \int_{-\infty}^{\infty} d q_{1} \frac{e^{-\theta\left(\frac{1}{\lambda}-i q_{1}-\epsilon\right)}-1}{\frac{1}{\lambda}-i q_{1}-\epsilon}\left\langle\overline{\tilde{x}\left(i q_{1}+\epsilon\right)}\right\rangle .
\end{aligned}
$$

A expressão para o primeiro cumulante da posição é obtido por:

$$
\begin{aligned}
\left\langle\overline{\tilde{x}\left(i q_{1}+\epsilon\right)}\right\rangle & =\left\langle\frac{\xi\left(i q_{1}+\epsilon\right)\left(\left(i q_{1}+\epsilon\right) \tau+1\right)}{R\left(i q_{1}+\epsilon\right)}+\frac{k_{2} L_{0}\left(\left(i q_{1}+\epsilon\right) \tau+1\right)}{\left(\left(i q_{1}+\epsilon\right)\left(\lambda\left(i q_{1}+\epsilon\right)+1\right) R\left(i q_{1}+\epsilon\right)\right.}\right\rangle \\
& =\frac{\left\langle\xi\left(i q_{1}+\epsilon\right)\right\rangle\left(\left(i q_{1}+\epsilon\right) \tau+1\right)}{R\left(i q_{1}+\epsilon\right)}+\frac{k_{2} L_{0}\left(\left(i q_{1}+\epsilon\right) \tau+1\right)}{\left(\left(i q_{1}+\epsilon\right)\left(\lambda\left(i q_{1}+\epsilon\right)+1\right) R\left(i q_{1}+\epsilon\right)\right.} \\
& =\frac{k_{2} L_{0}\left(\left(i q_{1}+\epsilon\right) \tau+1\right)}{\left(\left(i q_{1}+\epsilon\right)\left(\lambda\left(i q_{1}+\epsilon\right)+1\right) R\left(i q_{1}+\epsilon\right)\right.} .
\end{aligned}
$$

Substituindo o resultado encontrado em 4-33 na Eq. (4-32), e calculando a integral, obtemos o primeiro cumulante do trabalho $W$, que segue

$$
\begin{aligned}
\left\langle\overline{I_{\theta}}\right\rangle= & -\frac{k_{2}^{2} L_{0}^{2} e^{-\frac{\theta}{\lambda}}\left(\kappa_{1} \tau+1\right)\left(e^{\theta \kappa_{1}}-e^{\theta / \lambda}\right)}{m \kappa_{1}\left(\kappa_{1}-\kappa_{2}\right)\left(\kappa_{1}-\kappa_{3}\right)\left(\kappa_{1}^{2} \lambda^{2}-1\right)}- \\
& -\frac{k_{2}^{2} L_{0}^{2} e^{-\frac{\theta}{\lambda}}\left(\kappa_{2} \tau+1\right)\left(e^{\theta \kappa_{2}}-e^{\theta / \lambda}\right)}{m \kappa_{2}\left(\kappa_{2}-\kappa_{1}\right)\left(\kappa_{2}-\kappa_{3}\right)\left(\kappa_{2}^{2} \lambda^{2}-1\right)}- \\
& -\frac{k_{2}^{2} L_{0}^{2} e^{-\frac{\theta}{\lambda}}\left(\kappa_{3} \tau+1\right)\left(e^{\theta \kappa_{3}}-e^{\theta / \lambda}\right)}{m \kappa_{3}\left(\kappa_{3}-\kappa_{1}\right)\left(\kappa_{3}-\kappa_{2}\right)\left(\kappa_{3}^{2} \lambda^{2}-1\right)}- \\
& -\frac{\lambda^{2} k_{2}^{2} L_{0}^{2} e^{-\frac{2 \theta}{\lambda}}\left(e^{\frac{2 \theta}{\lambda}}-1\right)(\lambda-\tau)}{2 m\left(\kappa_{1} \lambda+1\right)\left(\kappa_{2} \lambda+1\right)\left(\kappa_{3} \lambda+1\right)}+ \\
& +\frac{k_{2}^{2} L_{0}^{2} e^{-\frac{\theta}{\lambda}}\left(e^{\theta / \lambda}-1\right)}{m \kappa_{1} \kappa_{2} \kappa_{3}} .
\end{aligned}
$$

\subsection{2}

\section{Segundo cumulante $\left\langle\overline{I_{\theta}^{2}}\right\rangle$}

De modo similar ao cálculo do primeiro cumulante do trabalho $W$, o segundo cumulante é obtido a partir da solução da seguinte integral: 


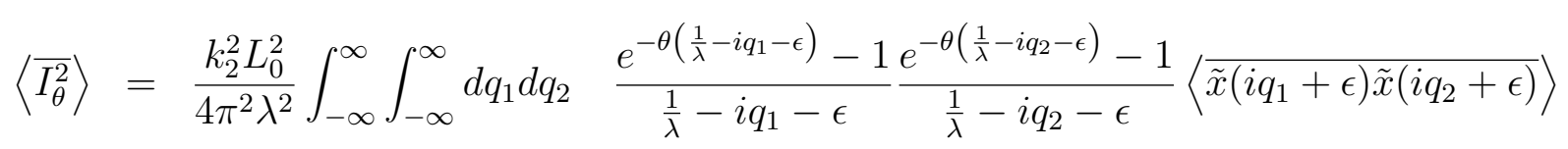

O segundo cumulante da posição é dado pela expressão:

$$
\left\langle\overline{I_{\theta}^{2}}\right\rangle=A_{1}+A_{2}+A_{3}+A_{4}+A_{5}
$$

Os termos $A_{i}$ estão expressos no apêndice B.5 por causa da sua extensão. Contudo, o procedimento é similar ao realizado para o primeiro cumulante: primeiramente calculamos o termo $\left\langle\overline{\tilde{x}\left(i q_{1}+\epsilon\right) \tilde{x}\left(i q_{2}+\epsilon\right)}\right\rangle$, e substituimos o resultado obtido na Eq. (4-35), calculamos a integral e obtemos o segundo cumulante do trabalho $W$.

\subsection{3}

\section{Igualdade de Jarzynski: caso linear}

Devido às características da distribuição gaussiana $\xi(t)$, somente o primeiro e segundo cumulante não são nulos e contribuem para a FGC. Portanto, substituindo $\left\langle\overline{I_{\theta}}\right\rangle$ e $\left\langle\overline{I_{\theta}^{2}}\right\rangle$ na Eq. (4-24), após algumas manipulações algébricas, obtemos:

$$
\ln G(u)=\ln \left\langle\overline{\exp \left[-i u W_{\theta}\right]}\right\rangle=-i u \Delta U-(i u)\left\langle\overline{I_{\theta}}\right\rangle+\frac{(-i u)^{2}}{2}\left\langle\overline{I_{\theta}^{2}}\right\rangle
$$

A IJ deve ocorrer quando $u=-\frac{i}{T}$. Assim, temos

$$
\ln G\left(-\frac{i}{T}\right)=\ln \left\langle\overline{\exp \left[\frac{-W_{\theta}}{T}\right]}\right\rangle=-\frac{k_{2} L_{\theta}^{2}}{2 T}-\frac{1}{T}\left\langle\overline{I_{\theta}}\right\rangle+\frac{1}{2 T^{2}}\left\langle\overline{I_{\theta}^{2}}\right\rangle .
$$

A variação da energia livre é dada por [65],

$$
\Delta F=\frac{L_{\theta}^{2}}{2} \frac{k_{1} k_{2}}{k_{1}+k_{2}} .
$$

E a Igualdade de Jarzynski é, então, escrita na forma

$$
\begin{aligned}
-\frac{k_{2} L_{\theta}^{2}}{2 T}-\frac{1}{T}\left\langle\overline{I_{\theta}}\right\rangle+\frac{1}{2 T^{2}}\left\langle\overline{I_{\theta}^{2}}\right\rangle & =-\frac{L_{\theta}^{2}}{2 T} \frac{k_{1} k_{2}}{k_{1}+k_{2}} \\
-\frac{k_{2} L_{0}^{2}\left(1-e^{-\frac{\theta}{\lambda}}\right)^{2}}{2 T}-\frac{1}{T}\left\langle\overline{I_{\theta}}\right\rangle+\frac{1}{2 T^{2}}\left\langle\overline{I_{\theta}^{2}}\right\rangle & =-\frac{L_{0}^{2}\left(1-e^{-\frac{\theta}{\lambda}}\right)^{2}}{2 T} \frac{k_{1} k_{2}}{k_{1}+k_{2}}
\end{aligned}
$$


Na qual, após substituirmos $\left\langle\overline{I_{\theta}}\right\rangle$ e $\left\langle\overline{I_{\theta}^{2}}\right\rangle$, verificamos exatamente a Igualdade de Jarzynski.

\subsection{4}

\section{Produção de entropia}

No segundo capítulo, apresentamos a conexão entre algumas técnicas da teoria da informação e a análise da termodinâmica de sistemas fora do equilíbrio. No contexto do nosso trabalho, vamos utilizar esta abordargem para calcular os possíveis valores máximos para o trabalho proveniente de um sistema submetido a um processo fora do equilíbrio.

Assim como nos modelos descritos anteriormente, vamos assumir que o sistema de interesse está constantemente em contato com o reservatório térmico à temperatura T. A entropia para o sistema fora do equilíbrio é dada pela Eq. (2-40), e tomando $k_{\beta}=1$, temos

$$
S(t)=-\int d x d v p(x, v, t) \ln p(x, v, t) .
$$

Neste ensejo, a energia interna do sistema de interesse é definida pela seguinte equação:

$$
U(t)=\int d x d v \rho(x, v, t) H(x, v)
$$

Portanto, podemos expressar a energia livre de Helmholtz fora do equilíbrio da seguinte forma:

$$
F(t)=U(t)-T S(t)
$$

A variação da entropia do reservatório é dada pelo valor negativo do fluxo de calor em direção ao sistema de interesse. Logo, podemos escrever:

$$
\Delta S_{R}(t)=-\frac{1}{T} \int_{0}^{t} d t^{\prime} j_{Q}\left(t^{\prime}\right)
$$

na qual $j_{Q}\left(t^{\prime}\right)$ é o fluxo de calor, definido na Eq. (4-16). Relembrando que o fluxo pode ser obtido facilmente multiplicando a Eq. (4-2) por $v(t)$, deixando em evidência a taxa de variação temporal da energia mecânica, e é expresso a seguir [80]:

$$
j_{Q}\left(t^{\prime}\right)=\xi\left(t^{\prime}\right) \dot{x}\left(t^{\prime}\right)-\int_{0}^{t^{\prime}} d t^{\prime \prime} \phi\left(t^{\prime}-t^{\prime \prime}\right) \dot{x}\left(t^{\prime \prime}\right) \dot{x}\left(t^{\prime}\right) .
$$

Já a variação da entropia do sistema de interesse é dado pela integral

$$
\Delta S(t)=-\int d x d v(p(x, v, t) \ln p(x, v, t)-p(x, v, 0) \ln p(x, v, 0)) .
$$

A variação da entropia total, para o sistema de interesse e reservatório térmico combinados, é a soma dos dois respectivos termos da variação da 
entropia definidos anteriormente, notadamente como segue:

$$
\Delta S_{t o t}=\Delta S(t)+\Delta S_{R}(t) .
$$

Relembrando a condição do caso linear $\left(k_{3}=0\right)$, vamos agora calcular de forma exata a variação de entropia do modelo proposto. Para isso, começamos expressando a Eq. (4-2) da seguinte forma:

$$
m \dot{v}(t)=-\int_{0}^{t} d t^{\prime} \phi\left(t-t^{\prime}\right) v\left(t^{\prime}\right)-k_{1} x(t)-k_{2}[x(t)-L(t)]+\xi(t) .
$$

No tempo inicial do protocolo $L(t=0)=0$, o sistema de interesse está em equilíbrio térmico com o reservatório à temperatura $T$. Daí a distribuição de probabilidade para as condições iniciais admite a equivalência $p_{0}\left(x_{0}, v_{0}\right) \equiv p\left(x_{0}, v_{0}, t=0\right)$. Então, de acordo com a Eq. (4-31), a distribuição de probabilidade para as condições iniciais tem características de uma distribuição gaussiana, descrita como segue:

$$
p_{0}\left(x_{0}, v_{0}\right)=\frac{\sqrt{\left(k_{1}+k_{2}\right) m}}{2 \pi T} \mathrm{e}^{-\frac{m v_{0}^{2}}{2 T}-\frac{\left(k_{1}+k_{2}\right) x_{0}^{2}}{2 T}}
$$

Devido à natureza linear da Eq. (4-48), podemos aplicar as ferramentas de função de Green para calcular a solução da equação (de forma similar à aplicação citada no capítulo anterior) [81, 65]. A solução é tomada em duas partes: a solução particular (a qual tem a função estocástica $\xi(t)$ e o protocolo $L(t)$ como os termos de fonte), e a solução homogênea (a qual depende das condições iniciais $x_{0}$ e $v_{0}$ ). As duas soluções combinadas são expressas a seguir:

$$
\begin{aligned}
& x(t)=\int_{0}^{t} d t^{\prime} G\left(t-t^{\prime}\right)\left[\xi\left(t^{\prime}\right)+k_{2} L\left(t^{\prime}\right)\right]+x_{0} f(t)+m v_{0} G(t), \\
& v(t)=\int_{0}^{t} d t^{\prime} \dot{G}\left(t-t^{\prime}\right)\left[\xi\left(t^{\prime}\right)+k_{2} L\left(t^{\prime}\right)\right]+x_{0} \dot{f}(t)+m v_{0} \dot{G}(t) .
\end{aligned}
$$

Na qual a função de Green $G(t)$ e a função auxiliar $f(t)$ são definidas abaixo:

$$
\begin{gathered}
G(t)=\lim _{\epsilon \rightarrow 0} \int_{-\infty}^{\infty} \frac{d q}{2 \pi} \frac{e^{(i q+\epsilon) t}}{\mathcal{R}(i q+\epsilon)} \\
f(t)=\lim _{\epsilon \rightarrow 0} \int_{-\infty}^{\infty} \frac{d q}{2 \pi} \frac{m(i q+\epsilon)+m \tilde{\phi}(i q+\epsilon)}{\mathcal{R}(i q+\epsilon)} e^{(i q+\epsilon) t} .
\end{gathered}
$$


O termo $\mathcal{R}(s)$ pode ser expresso de forma mais compacta como segue:

$$
\begin{aligned}
\mathcal{R}(s) & =m s^{2}+\frac{\gamma s}{1+\tau s}+k_{1}+k_{2}=\frac{m \tau s^{3}+(m+\tau \gamma) s^{2}+\tau\left(k_{1}+k_{2}\right) s+k_{1}+k_{2}}{1+\tau s} \\
& =\frac{m\left(s-\kappa_{1}\right)\left(s-\kappa_{2}\right)\left(s-\kappa_{3}\right)}{1+\tau s} .
\end{aligned}
$$

Assim, $\mathcal{R}(s)$ fica em função das raízes $\kappa_{1}, \kappa_{2}$ e $\kappa_{3}$.

Os coeficientes do polinômio $\mathcal{R}(s)$ são reais e positivos. Portanto, vamos utilizar o discriminante $\Delta$ da conhecida solução para uma equação do terceiro grau [82]. É o discriminante $\Delta$ que determinará se as raízes são todas reais $(\Delta>0)$, todas reais com duas idênticas $(\Delta=0)$ ou uma das raízes é real e duas raízes são complexas, sendo uma o complexo conjugado da outra $(\Delta<0)$. O discriminante é dado pela equação a seguir:

$$
\Delta=\Gamma^{2}-4 \Gamma^{3} \tau-4 \omega^{2}+4 \Gamma \tau(5-3 \Gamma \tau) \omega^{2}-4 \tau^{2}(2+3 \Gamma \tau) \omega^{4}-4 \tau^{4} \omega^{6},
$$

na qual $\Gamma=\gamma / m$ e $\omega^{2}=\left(k_{1}+k_{2}\right) / m$. Quando $\tau=0$, o discriminante toma a forma $\Delta=\Gamma^{2}-4 \omega^{2}$, o qual ocorre quando a força estocástica da Eq. (4-48) é um ruído branco.

Sabendo disso, podemos reescrever a função de Green da Eq. (4-52). Quando o discriminante $\Delta$ é positivo, temos:

$$
\begin{aligned}
G(t) & =\frac{\kappa_{1} \tau-1}{m\left(\kappa_{1}-\kappa_{2}\right)\left(\kappa_{1}-\kappa_{3}\right)} e^{-\kappa_{1} t}+\frac{\kappa_{2} \tau-1}{m\left(\kappa_{2}-\kappa_{1}\right)\left(\kappa_{2}-\kappa_{3}\right)} e^{-\kappa_{2} t}+ \\
& +\frac{\kappa_{3} \tau-1}{m\left(\kappa_{3}-\kappa_{1}\right)\left(\kappa_{3}-\kappa_{2}\right)} e^{-\kappa_{3} t}
\end{aligned}
$$

Se o discriminante for negativo, as raízes tomam a forma $\left(\kappa_{2,3}=\kappa_{R}+ \pm i \kappa_{I}\right)$, na qual $\kappa_{1}=\kappa_{R}$ é real e temos as frequências de oscilação $\operatorname{Im}\left(\kappa_{2}\right)=\operatorname{Im}\left(\kappa_{3}\right)=\kappa_{I}$. Neste caso, a função de Green é dada por:

$$
\begin{aligned}
G(t) & =\frac{\left(1-\kappa_{1} \tau\right) e^{-\kappa_{1} t}}{m\left(\kappa_{I}^{2}+\left(\kappa_{1}-\kappa_{R}\right)^{2}\right)}+\frac{\left(\kappa_{1} \tau-1\right) e^{-\kappa_{R} t}}{m\left(\kappa_{I}^{2}+\left(\kappa_{1}-\kappa_{R}\right)^{2}\right)} \cos \left(\kappa_{I} t\right)+ \\
& +\frac{\left(\kappa_{1}-\kappa_{R}-\kappa_{1} \kappa_{R} \tau+\left(\kappa_{I}^{2}+\kappa_{R}^{2}\right) \tau\right) e^{-\kappa_{R} t}}{m\left(\kappa_{I}^{2}+\left(\kappa_{1}-\kappa_{R}\right)^{2}\right)} \operatorname{sen}\left(\kappa_{I} t\right) .
\end{aligned}
$$

Do mesmo modo, quando o discriminante $\Delta$ for positivo, a função auxiliar $f(t)$ toma a forma: 


$$
\begin{aligned}
f(t) & =\frac{\kappa_{1}^{2} \tau-\kappa_{1}+\Gamma}{m\left(\kappa_{1}-\kappa_{2}\right)\left(\kappa_{1}-\kappa_{3}\right)} e^{-\kappa_{1} t}+\frac{\kappa_{2}^{2} \tau-\kappa_{2}+\Gamma}{m\left(\kappa_{2}-\kappa_{1}\right)\left(\kappa_{2}-\kappa_{3}\right)} e^{-\kappa_{2} t}+ \\
& +\frac{\kappa_{3}^{2} \tau-\kappa_{3}+\Gamma}{m\left(\kappa_{3}-\kappa_{1}\right)\left(\kappa_{3}-\kappa_{2}\right)} e^{-\kappa_{3} t}
\end{aligned}
$$

E se o discriminante for negativo, temos:

$$
\begin{aligned}
f(t) & =\frac{\kappa_{1}^{2} \tau-\kappa_{1}+\Gamma}{m\left(\kappa_{1}-\kappa_{2}\right)\left(\kappa_{1}-\kappa_{3}\right)} e^{-\kappa_{1} t}+\frac{\left(\Gamma+\kappa_{1}+\left(\kappa_{I}+\kappa_{R}\left(\kappa_{T}-2 \kappa_{1}\right) \tau\right)\right) e^{-\kappa_{R} t}}{m\left(\kappa_{I}^{2}+\left(\kappa_{1}-\kappa_{R}\right)^{2}\right)} \cos \left(\kappa_{I} t\right)+ \\
& +\frac{\left(\Gamma+\kappa_{1}+\left(\kappa_{I}^{2}+\kappa_{R}\left(\kappa_{R}-2 \kappa_{1}\right)\right) \tau\right) e^{-\kappa_{R} t}}{m\left(\kappa_{I}^{2}+\left(\kappa_{1}-\kappa_{R}\right)^{2}\right)} \operatorname{sen}\left(\kappa_{I} t\right) .
\end{aligned}
$$

Para compormos a distribuição instantânea $p(x, v, t)$ para a posição e a velocidade, precisamos calcular os valores esperados e as variâncias de $x(t) \mathrm{e}$ $v(t)$. O valor esperado é obtido diretamente, um vez que $\langle\xi(t)\rangle=0$. Assim, podemos escrever:

$$
\begin{aligned}
& \mu_{x}(t)=\langle\overline{x(t)}\rangle=\int_{0}^{t} d t^{\prime} G\left(t-t^{\prime}\right) k_{2} L\left(t^{\prime}\right)+\left\langle x_{0}\right\rangle f(t)+\left\langle v_{0}\right\rangle m G(t)(4-60) \\
& \mu_{v}(t)=\langle\overline{v(t)}\rangle=\int_{0}^{t} d t^{\prime} \dot{G}\left(t-t^{\prime}\right) k_{2} L\left(t^{\prime}\right)+\left\langle\overline{x_{0}}\right\rangle \dot{f}(t)+\left\langle\overline{v_{0}}\right\rangle m \dot{G}(t) .(4-61)
\end{aligned}
$$

Os valores esperados de $\overline{x_{0}}$ e $\overline{v_{0}}$ são nulos devido à natureza gaussiana da distribuição 4-49.

As variâncias são definidas pelas equações:

$$
\begin{aligned}
\sigma_{x x}(t) & =\langle x(t) x(t)\rangle-\mu_{x}(t)^{2}, \\
\sigma_{v v}(t) & =\langle v(t) v(t)\rangle-\mu_{v}(t)^{2}, \\
\sigma_{x v}(t) & =\langle x(t) v(t)\rangle-\mu_{x}(t) \mu_{v}(t) .
\end{aligned}
$$

Deste modo, lidamos com um pouco mais de álgebra, resultando, por fim, nas seguintes expressões: 


$$
\begin{aligned}
\sigma_{x x}(t) & =\int_{0}^{t} d t_{1} d t_{2} G\left(t-t_{1}\right) G\left(t-t_{2}\right)\left\langle\xi\left(t_{1}\right) \xi\left(t_{2}\right)\right\rangle+ \\
& +\left\langle x_{0}^{2}\right\rangle f^{2}(t)+\left\langle v_{0}^{2}\right\rangle m^{2} G^{2}(t) \\
\sigma_{v v}(t) & =\int_{0}^{t} d t_{1} d t_{2} \dot{G}\left(t-t_{1}\right) \dot{G}\left(t-t_{2}\right)\left\langle\eta\left(t_{1}\right) \eta\left(t_{2}\right)\right\rangle+ \\
& +\left\langle x_{0}^{2}\right\rangle \dot{f}^{2}(t)+\left\langle v_{0}^{2}\right\rangle m^{2} \dot{G}^{2}(t) \\
\sigma_{x v}(t) & =\int_{0}^{t} d t_{1} d t_{2} G\left(t-t_{1}\right) \dot{G}\left(t-t_{2}\right)\left\langle\eta\left(t_{1}\right) \eta\left(t_{2}\right)\right\rangle+ \\
& +\left\langle x_{0} v_{0}\right\rangle m\{f(t) \dot{G}(t)+\dot{f}(t) G(t)\} .
\end{aligned}
$$

Coletando os resultados dos valores esperados e das variâncias para a posição e a velocidade, podemos construir a distribuição de probabilidade instantânea, que é dada por [83]:

$$
\begin{aligned}
p(x, v, t) & =\frac{1}{2 \pi \sqrt{\sigma_{x x} \sigma_{v v}-\sigma_{x v}^{2}}} \times \\
& \times \exp \left\{-\frac{1}{2} \frac{\sigma_{v v}\left(x-\mu_{x}\right)^{2}+\sigma_{x x}\left(v-\mu_{v}\right)^{2}-2 \sigma_{x v}\left(x-\mu_{x}\right)\left(v-\mu_{v}\right)}{\sigma_{x x} \sigma_{v v}-\sigma_{x v}^{2}}\right\} .
\end{aligned}
$$

Assim, substituindo as Eqs. (4-49) e 4-68 na definição da variação da entropia do sistema de interesse da Eq.( 4-46), calculamos a integral e obtemos a seguinte expressão na sua forma exata:

$$
S(t)=\frac{1}{2} \ln \left(\sigma_{x x}(t) \sigma_{v v}(t)-\sigma_{x v}^{2}(t)\right)+\ln 2 \pi e .
$$

Devido ao fato de que as variâncias $\sigma_{x x}, \sigma_{x x}$ e $\sigma_{x x}$ não dependem de $L_{0}$ ou $\lambda$, a entropia do sistema de interesse também não depende desses parâmetros neste modelo linear não markoviano. Também, o valor $\sigma_{x x}(t) \sigma_{v v}(t)-\sigma_{x v}^{2}(t)$ é positivo, isto garante que a entropia $S(t)$ seja bem definida. Assim, podemos expressar essa característica do sistema de interesse pela expressão:

$$
\frac{\partial S}{\partial L_{0}}=\frac{\partial S}{\partial \lambda}=0
$$

Este comportamento não usual da entropia é predominantemente devido ao comportamento do termo $\sigma_{x v}(t)$ (transiente), cujo valor é zero no equilíbrio. As correlações da posição e velocidade são estreitamente ligadas ao modos hidrodinâmicos lentos, que surgem em um fluido perturbado pelo movimento de uma partícula browniana, o que justifica a função de memória dissipativa 


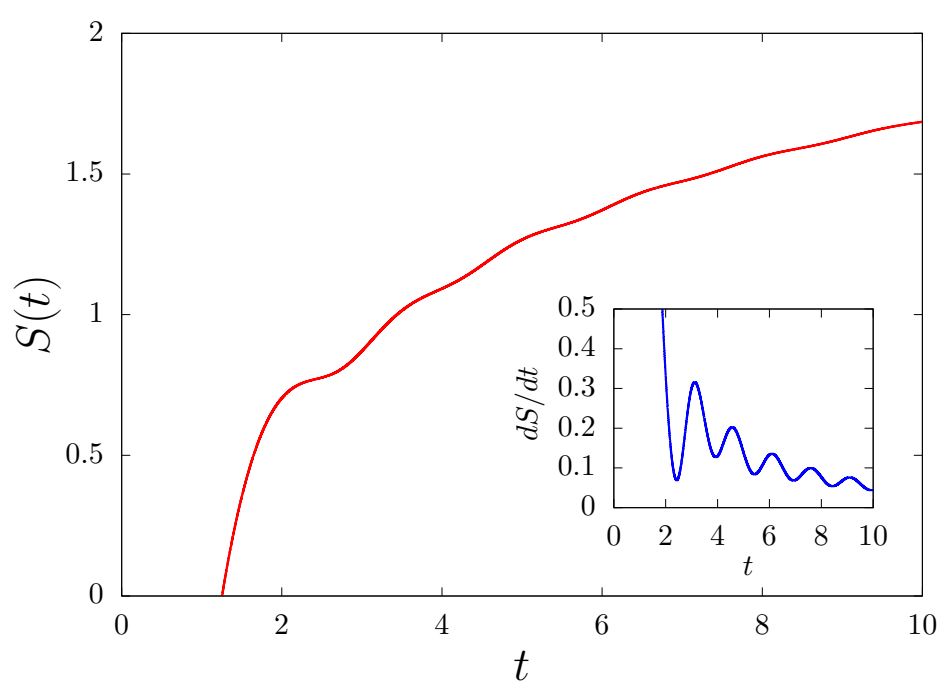

Figura 4.1: Evolução da entropia de uma partícula Browniana com as condições iniciais $x_{0}=v_{0}=0$. A taxa de variação da entropia se aproxima de valores negativos, ainda que fosse esperado um crescimento monotônico da entropia neste caso.

presente neste modelo não markoviano [84]. Na Figura (4.1) podemos observar a evolução da entropia para este sistema em direção do equilíbrio, e o comportamento não usual da flutuação da taxa de variação da entropia. Em todas as simulações do modelo linear, os valores dos parâmetros utilizados foram: $k_{1}=k_{2}=2, \gamma=0,5, \tau=m=1$.

Para o modelo harmônico não-Markoviano linear da Eq. (4-48), com as condições iniciais dadas pela distribuição da Eq. (4-49), pode ser verificado que na ausência do protocolo $\left(L_{0}=0\right)$ o sistema se manterá no estado de equilíbrio e não haverá variação da entropia. Vamos analisar agora se o efeito de um protocolo não nulo é o suficiente para a produção de entropia no sistema combinado (reservatório, sistema de interesse e sistema externo).

Primeiramente, vamos demonstrar que a variação da entropia do sistema de interesse só pode ser produzida por ele mesmo. Embora a entropia possa ser transferida do reservatório para o sistema de interesse, e vice-versa, o reservatório não produz entropia, devido ao fato que se mantém constantemente no estado de equilíbrio. Em contraste com os reservatórios atérmicos, como os reservatórios de Poisson, que produzem entropia continuadamente [85, 86]. Ainda, o sistema externo não produz entropia e, também, não transfere entropia para o sistema de interresse. Daí concluimos que a entropia total (reservatório, sistema de interesse e sistema externo combinados) é igual a entropia produzida no sistema de interesse.

Em segundo lugar, vamos expressar a produção total da entropia nesta forma: 


$$
\Delta S_{t o t}(t)=\Delta S(t)+\Delta S_{R}(t)=\Pi_{S}(t),
$$

na qual $\Pi_{S}(t)$ é a entropia total produzida no sistema de interesse durante o intervalo $0 \rightarrow t$.

A única interferência que pode conduzir o sistema para fora do equilíbrio é a atuação do protocolo da força externa $L(t)$. É somente pela ação do protocolo que se poderia produzir entropia no sistema de interesse, durante a sua evolução temporal.

Como demonstrado na Eq. (4-70), a entropia $S(t)$, calculada para o sistema de interesse, é independente dos parâmetros do protocolo. Então, esta relação também dever ser válida para um processo quase-estático $(\lambda \rightarrow \infty)$. Logo, o sistema de interesse está sempre em equilíbrio, sem nenhuma produção de entropia, o que nos permite escrever:

$$
\Pi_{S}(t \geq 0)=0 .
$$

Por fim, se quisermos produzir entropia durante a atuação do protocolo, é essencial a presença de um potencial não harmônico atuando sobre o sistema de interesse. Analisaremos numericamente esta situação posteriormente, mas antes vamos estudar as oscilações desta entropia.

\subsection{5}

\section{Oscilações da entropia}

A fim de analisarmos o comportamento das Eqs. (4-68) e (4-69), vamos comparar os resultados analíticos para a entropia, detalhados na última seção, com a integração numérica da Eq. 4-48. Para isso, supomos a seguinte situação: o sistema parte das condições iniciais $x_{0}=0$ e $v_{0}=0$, evoluindo de acordo com o protocolo $L(t)$, até atingir o equilíbrio quando $t \rightarrow \infty$. Neste caso, todos os cumulantes inicialmente são nulos e evoluem na direção dos valores de equilíbrio:

$$
\begin{aligned}
\mu_{v}(\infty) & =0 \\
\mu_{x}(\infty) & =L_{0} / 2=0,5 \\
\sigma_{x x}(\infty) & =1,0 \\
\sigma_{v v}(\infty) & =0,25 \\
\sigma_{v x}(\infty) & =0
\end{aligned}
$$

A Figura 4.2 representa o processo de termalização do sistema de interesse. A frequência observada em (a) é a mesma, tanto para $\mu_{x}$ quanto 


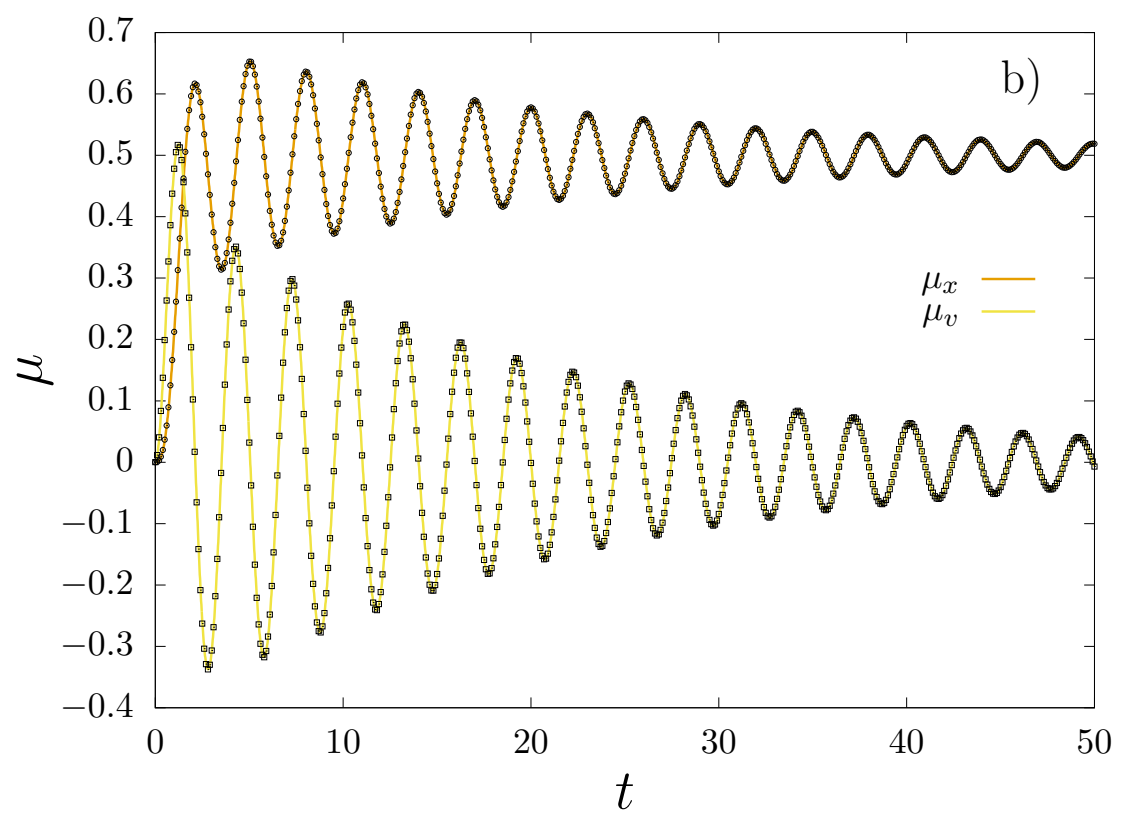

(a)

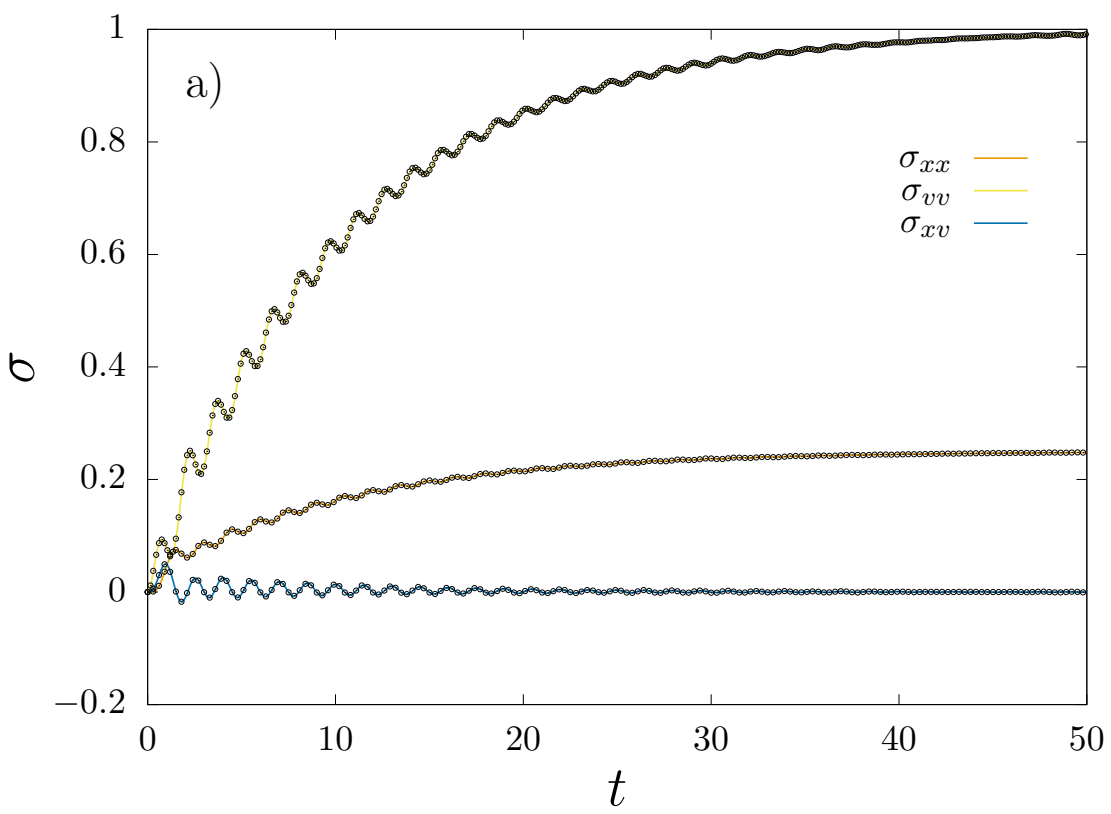

(b)

Figura 4.2: Comparação dos resultados numéricos (pontos) com as predições teóricas (linhas) para os valores esperados $\mu$ (a) e para as variâncias $\sigma$ (b) definidos na última seção. Para as integrações numéricas, foram escolhidos $k_{1}=k_{2}=2, \gamma=0,5$ e $m=\tau=T=\lambda=L_{0}=1$, com $x_{0}=v_{0}=0$, para os valores dos parâmetros. 
para $\mu_{v}$, e seu valor é determinado pela raiz imaginária $\left(\kappa_{I} \simeq 2\right)$. Em (b), a frequência de oscilação é uma combinação de $\kappa_{I}$ e $2 \kappa_{I}$, dominada pela última raiz, e é proporcional à segunda potência dos termos $G(t)$ e $f(t)$, em harmonia com as predições teóricas.

Uma característica interessante do comportamento das variâncias, evoluindo em direção ao equilíbrio, é que as oscilações são consequência direta da função memória $\phi\left(t-t^{\prime}\right)$ e, portanto, do tempo característico $(\tau \neq 0)$. Ainda, as oscilações da variância, e da entropia por sua dependência, representam um fluxo oscilante de informação entre o sistema de interesse e o reservatório térmico. No caso da Figura 4.2, a energia inicial do sistema é nula, isto implica que o calor tenha um fluxo do reservatório em direção ao sistema, causando o aumento da entropia do último. Contudo, deixando o sistema de interesse evoluir por um período suficiente de tempo, o fluxo de informação da entropia inverte seu sentido. A informação é recuperada do reservatório por meio da função memória.

Para que o sistema de interesse produza entropia, é necessário conduzir o sistema para um estado fora do equilíbrio, ou seja, realizar um processo que não seja quase-estático sobre ele. O protocolo apresentado anteriomente para o modelo proposto possui essa característica, a não ser que a taxa do protocolo seja quase-estática (quando $\lambda \rightarrow 0$ ), e isto deveria ser o suficiente para acessar um estado fora do equilíbrio. Entretanto, neste caso isto não ocorre: para um modelo harmônico não markoviano, com as condições iniciais de equilíbrio dada pela distibuição de Gibbs, dado que $L(t=0)=0$, o sistema de interesse permanece no estado de equilíbrio e nenhuma entropia é produzida.

Na Figura (4.3) (a), mostramos a evolução da entropia para uma partícula browniana com condições iniciais fixadas, termalizada a diferentes temperaturas iniciais. O gráfico inserido mostra a evolução da taxa de variação da entropia. Ainda que a taxa de variação tenha um comportamento oscilatório, uma vez que $G(t)$ e $f(t)$ oscilam, a entropia ainda é uma função monotônica, crescendo em direção ao equilíbrio, enquando $d S / d t \geq 0$ para qualquer tempo. Os parâmetros utilizados são $\gamma=0,5, k_{1}=k_{2}=2$ e $T_{f}=m=\lambda=L_{0}=\tau=1$.

O principal efeito da função memória é suprir o presente com informações do passado, disto que se dá a propriedade não markoviana do modelo. Um possível resultado de um modelo não markoviano deveria ser um fluxo reverso de informação, definido como $d S / d t<0$. Este é o caso deste modelo, porém, o modelo não markoviano nem sempre conduz a um fluxo reverso de informação.

Neste modelo, também há outro efeito interessante identificado como "oscilações da entropia", no qual podemos reconhecer as características de um típico comportamento oscilatório da evolução temporal da entropia $S(t)$. As 


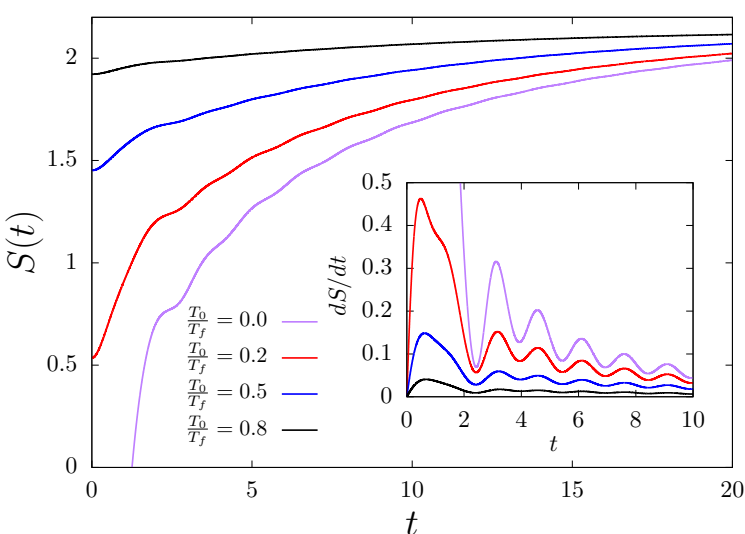

a)

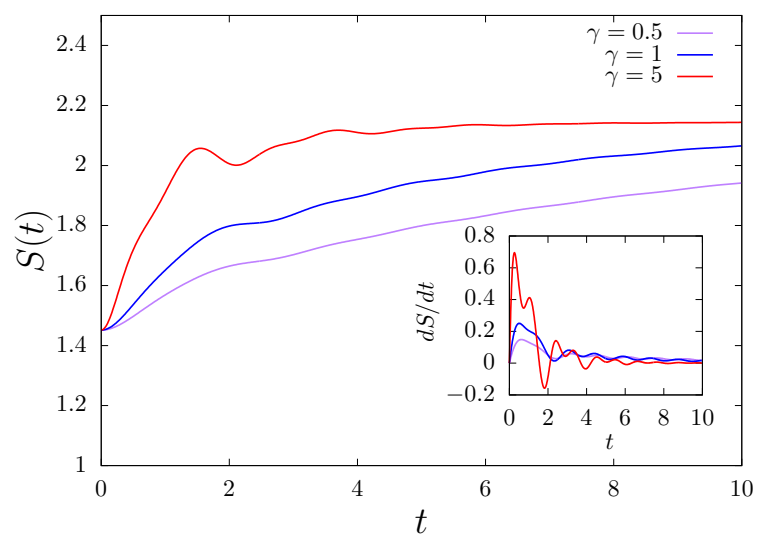

Figura 4.3: A evolução da entropia de uma partícula browniana, com condições iniciais fixadas, a diferentes temperaturas iniciais $T_{0}$. $\mathrm{O}$ gráfico inserido mostra a taxa de variação da entropia. Apesar da oscilação de $g(t)$ e $f(t)$, a entropia permanece sendo uma função monotônica (a). Em (b), apresentamos a evolução da entropia para diferentes valores da constante de amortecimento $\gamma$, enquanto que o gráfico inserido mostra a taxa de variação da entropia. Para valores suficientementes grandes de $\gamma$, o sistema apresenta fluxo reverso de informação, fazendo com que a entropia não seja mais uma função monotônica.

oscilações se apresentam nos regimes de alta e baixa dissipação, sendo ausentes para uma taxa intermediária de intensidade de dissipação.

Analisamos alguns cenários, mostrados na Figura (4.3) (b), no qual o sistema começa termalizado à temperatura $T_{0}$, que pode ser escolhida arbitrariamente. Observamos que, para valores grandes o suficiente para o coeficiente de dissipação $\gamma$, a taxa de variação da entropia se torna negativa, apresentando oscilações com um alacance temporal muito maior que o tempo característico de memória $\tau=1$. A função memória atua como uma bomba de informação, recuperando parcialmente algumas informações perdidas para o reservatório devido ao fluxo reverso de informação $d S / d t<0$. Este efeito diminui gradualmente na medida que o sistema alcaça se aproxima do equilíbrio, o que pode ser visto claramente no gráfico inserido na Figura (4.3) (b).

O espectro da variação da entropia da Figura(4.3) (b) pode ser observado 


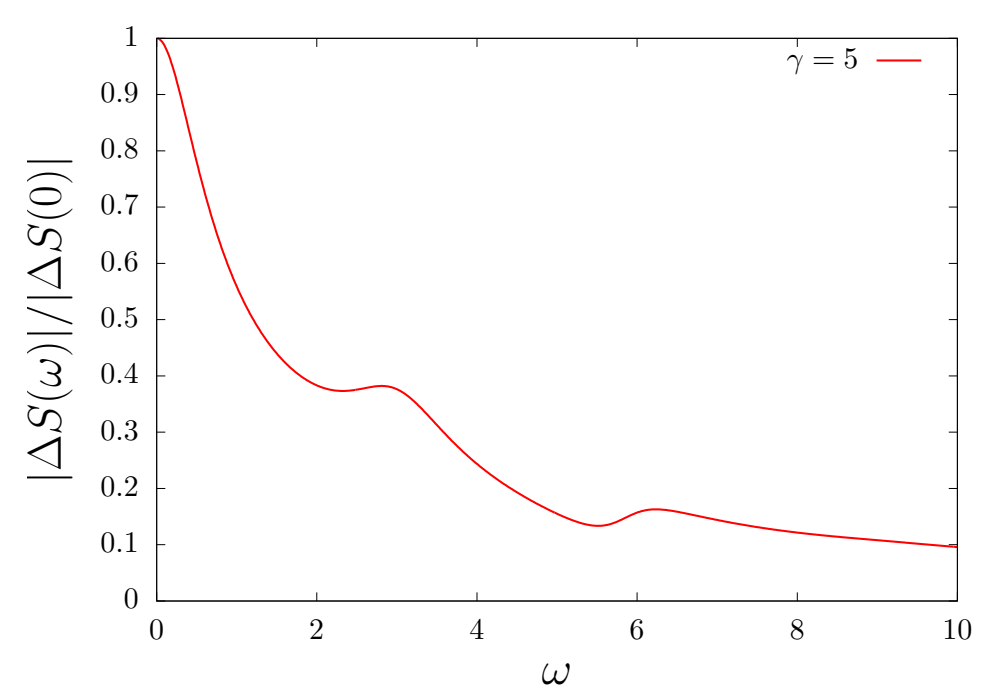

Figura 4.4: O espectro da entropia da Figura (4.3) (b) apresenta um pico acentuado em $\omega=0$, o que sugere o quase constante comportamento de $S(t)$.

na Figura (4.4), no qual são visíveis os picos pertencentes à série harmônica gerada por $\kappa_{I}=2,945$, em aproximadamente 3 e 6 . Os valores reais dos picos não são múltiplos exatos de $\kappa_{i}$. A análise espectral da entropia como uma função do tempo revela o comportamento oscilante das variâncias explicitamente. No Apêndice C.4, estudamos o comportamento das raízes $\kappa_{i}$.

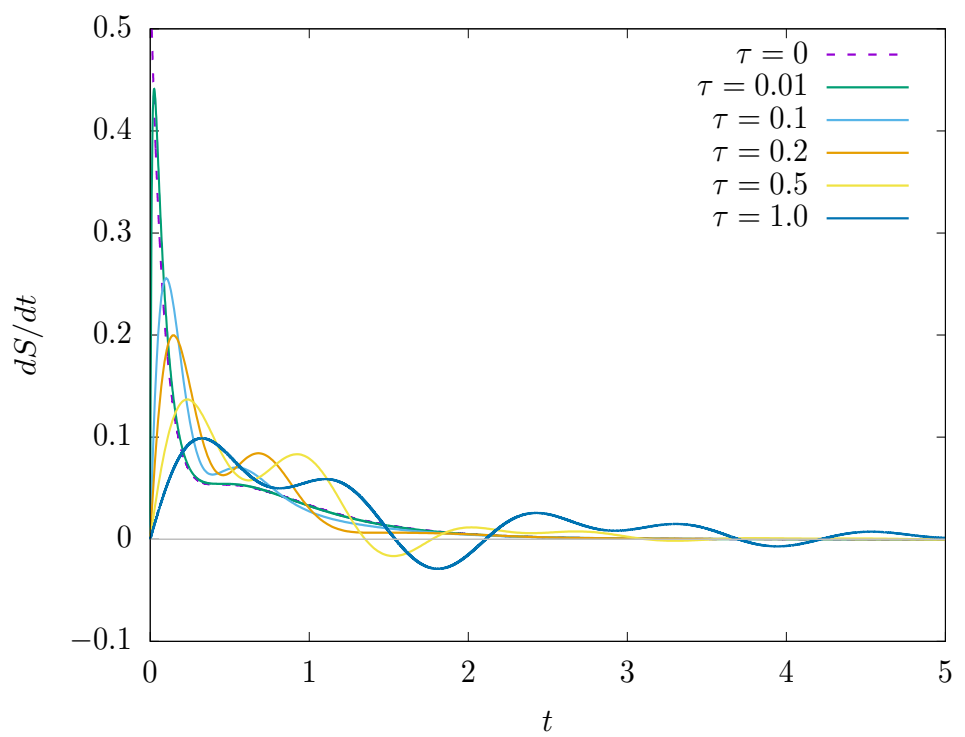

Figura 4.5: Comportamento da derivada temporal da entropia. Os parâmetros utilizados são $\tau=0,0.01,0.1,0.2,0.5,1.0, \gamma=5$ e $m=1$. Observe que para $\tau=0$, temos $d S / d t>0$, portanto, não há fluxo reverso de informação..

A presença das oscilações devido a $\kappa_{I}$ não garantem que haja o fluxo reverso de informação. Há outros fatores que contribuem para que a entropia 
descresça ou não. A fim de entendermos este ponto, vamos analisar o gráfico da Figura (4.5). Por exemplo, se $\tau=0$, o ruído Gaussiano será um ruído branco, e a entropia não diminuirá independentemente de $\kappa_{I} \neq 0$, considerando o protocolo aplicado a este modelo. O fluxo reverso de informação é a manifestação direta do acoplamento do núcleo de memória não markoviano com um regime de alta dissipação.

Como mostrado na Figura (4.5), testamos a existência do fluxo reverso de informação para diversos valores, acima e abaixo de $\tau_{c}$. Nós observamos que: para $\tau=0$, como esperado, nenhum fluxo reverso de informação é apresentado; para $\tau>\tau_{c}=0,05$, observamos muitas ocorrências de fluxo reverso de informação. Entretanto, para $\tau=0,2$, não há fluxo reverso de informação. Portanto, mesmo para grandes valores do coefiente de dissipação, ou equivalentemente para grandes valores de $\tau$, a ocorrência do fluxo reverso não é garantida.

Na próxima seção, vamos estudar o caso não linear $\left(k_{3} \neq 0\right)$, no qual a função memória também tem um papel central para a recuperação parcial de informação que inicialmente é perdida em forma de calor para o reservatório, ainda que o processo seja irreversível.

\section{3}

\section{Análise numérica para o caso não linear $\left(k_{3} \neq 0\right)$}

A análise do modelo não linear foi realizada pela simulação computacional do processo. O modelo linear foi generalizado adicionando o termo do potencial quártico $\left(k_{3} x^{4} / 4\right)$ à interação, evoluindo de acordo com a Eq. (4-2). A análise demonstrada nesta seção serve para embasar uma possível futura comparação com o resultado analítico do caso não linear. Os valores escolhidos para os parâmetros obedecem a relação $k_{3} / k_{1}^{2} \ll 1$. Assim, o potencial quártico pode ser considerado uma pequena correção para o potencial harmônico.

Os valores dos parâmetros utilizados para executar as simulações do processo são: $m=1,0, k_{1}=2,0, k_{2}=2,0, k_{3}=0,005, L_{0}=1,0, \tau=$ $1,0, T=1,0, \gamma=0,5$ e $\lambda=1,0$. O número de execuções do processo foi de 200.000 repetições, conduzidas de acordo com o protocolo $L(t)$. Os resultados apresentados a seguir são as médias tomadas das repetições da simulação. As condições iniciais são obtidas da distribuição de probabilidade da Eq. (4-49).

\subsection{1}

\section{Análise do calor}

Podemos separar o calor total $(Q(t))$ trocado com o reservatório até o tempo $t$ em duas partes: calor injetado $J_{i n j}(t)$ e o calor dissipado $J_{\text {diss }}(t)$, 
definidos na sequência:

$$
\begin{aligned}
Q(t) & =\Delta J(t) \\
& =J_{i n j}(t)+J_{\text {diss }}(t) .
\end{aligned}
$$

Na qual

$$
\begin{aligned}
J_{i n j}(t) & =\int_{0}^{t} d t^{\prime} \xi\left(t^{\prime}\right) v\left(t^{\prime}\right) \\
J_{\text {diss }}(t) & =-\int_{0}^{t} d t^{\prime} \int_{0}^{t^{\prime}} d t^{\prime \prime} \phi\left(t^{\prime}-t^{\prime \prime}\right) v\left(t^{\prime \prime}\right) v\left(t^{\prime}\right) .
\end{aligned}
$$

Na Figura (4.6) é possível observar o comportamento médio das funções $J_{i n j}(t)$ (linha azul contínua) e $J_{d i s s}(t)$ (linha tracejada vermelha) durante a evolução do processo nas simulações.

Na Figura (4.7), podemos observar certa tendência da troca de calor total $(\Delta J(t))$ saturar em torno de um valor negativo. Isto acontece devido à dinâmica do trabalho realizado sobre o sistema de interesse durante o protocolo: parte do trabalho é transferido para o reservatório na forma de calor.

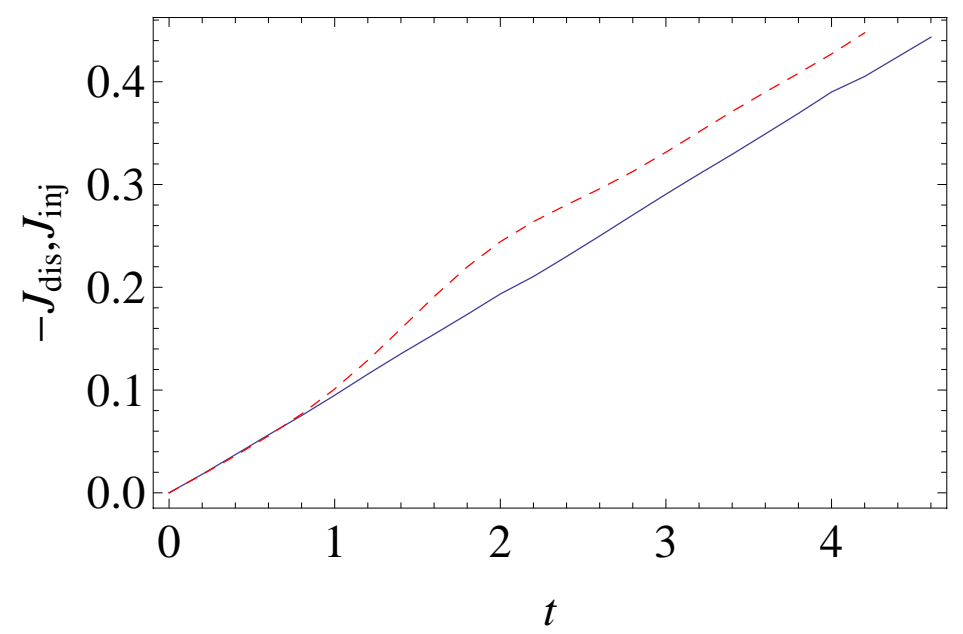

Figura 4.6: Calor injetado (linha azul contínua) e calor dissipado (linha tracejada vermelha) durante o protocolo $L(t)$.

Também podemos obter a distribuição de probabilidade para o calor injetado $p\left(J_{i n j}\right)$, durante a atuação do protocolo $L(t)$. Como mostrado na Figura (4.8), o decaimento exponencial revela uma relação com o teorema de flutuação para o calor injetado, análogo à relação da Eq. (2-36) derivada no segundo capítulo deste trabalho, a saber [87]: 


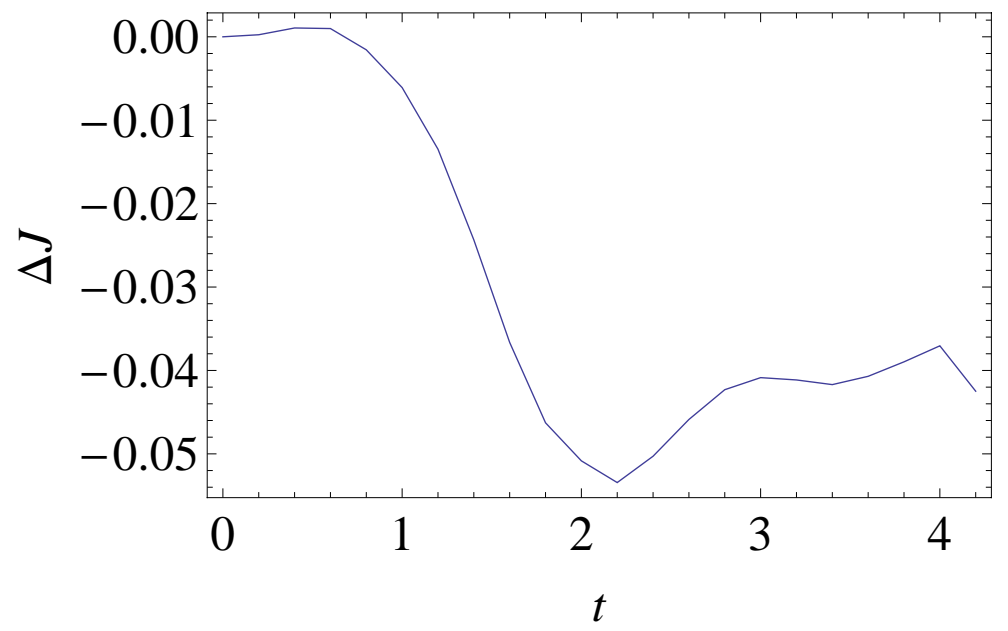

Figura 4.7: Calor total absorvido $\left(J_{\text {inj }}(t)+J_{\text {diss }}(t)\right)$ pelo sistema durante o protocolo $L(t)$.

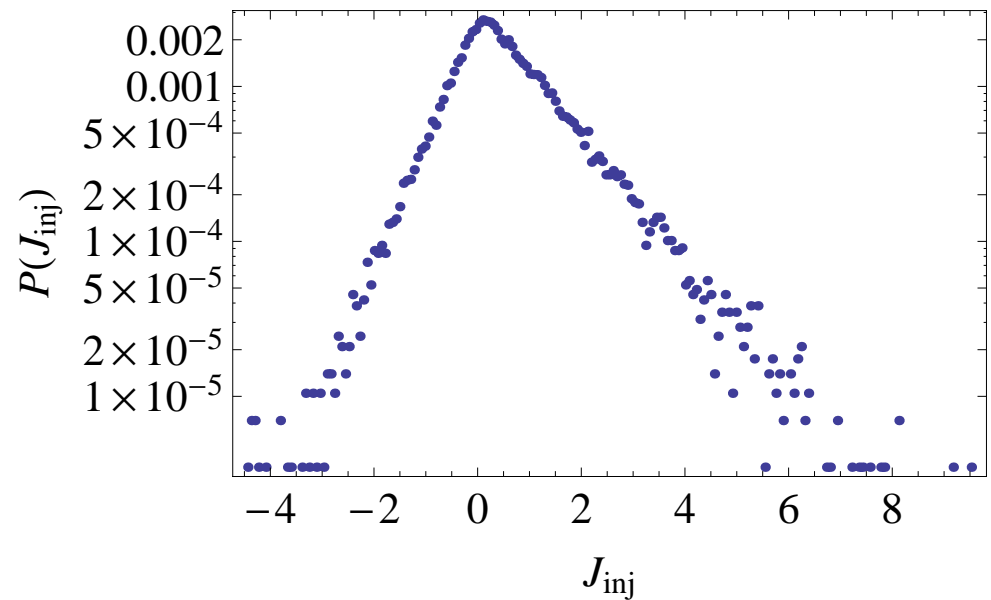

Figura 4.8: A distribuição de probabilidade para o calor injetado $J_{i n j}$.

$$
\ln \left(\frac{p\left(J_{i n j}\right)}{p\left(-J_{i n j}\right)}\right) \propto J_{i n j}
$$

O comportamento de proporcionalidade da Eq. (4-76) é verificado na Figura (4.9). Esta característica da distribuição já foi observada para sistemas com diferentes configurações, e podem ser conferidas em [61, 86].

\subsection{2}

\section{Análise do trabalho}

A distribuição de probabilidade para o trabalho tem uma notável assimetria em relação a $W=0$, como pode ser verificado na Figura (4.10). Por isso, claramente o valor esperado do trabalho externo é positivo $\left(\left\langle W_{\text {ext }}\right\rangle>0\right)$, pois a distribuição é enviesada na direção positiva do eixo do trabalho $W$. Este resultado é esperado porque corrobora com o processo conduzido pelo protocolo: o trabalho é realizado ao se esticar a mola $(L(0)=0 \rightarrow L(t)>0)$. 


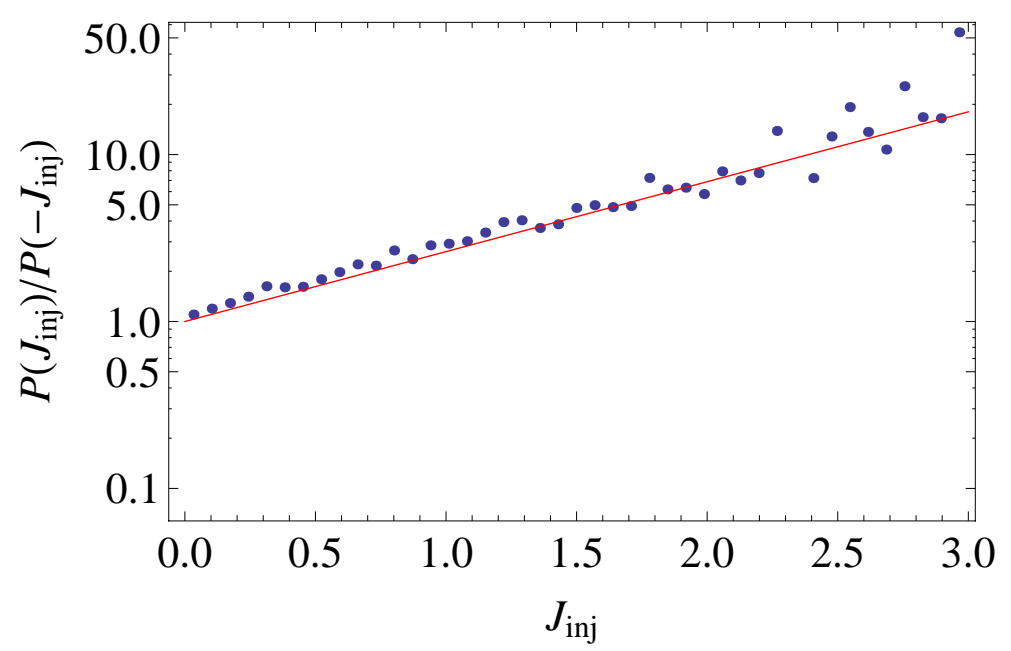

Figura 4.9: As flutuações do calor injetado obedecem ao respectivo teorema de flutuação.

A distribuição da Figura (4.10) tem características de uma gaussiana. Este mesmo comportamento pode ser encontrado no modelo similar descrito no terceiro capítulo deste trabalho [65]. A distribuição também revela uma relação com o respectivo teorema de flutuação, expresso na Eq. (2-37), a saber $[50]$ :

$$
\ln \left(\frac{p(W)}{p(-W)}\right) \propto W .
$$

Esta proporcionalidade pode ser verificada na Figura (4.14) e corrobora o comportamento gaussiano da distribuição do trabalho.

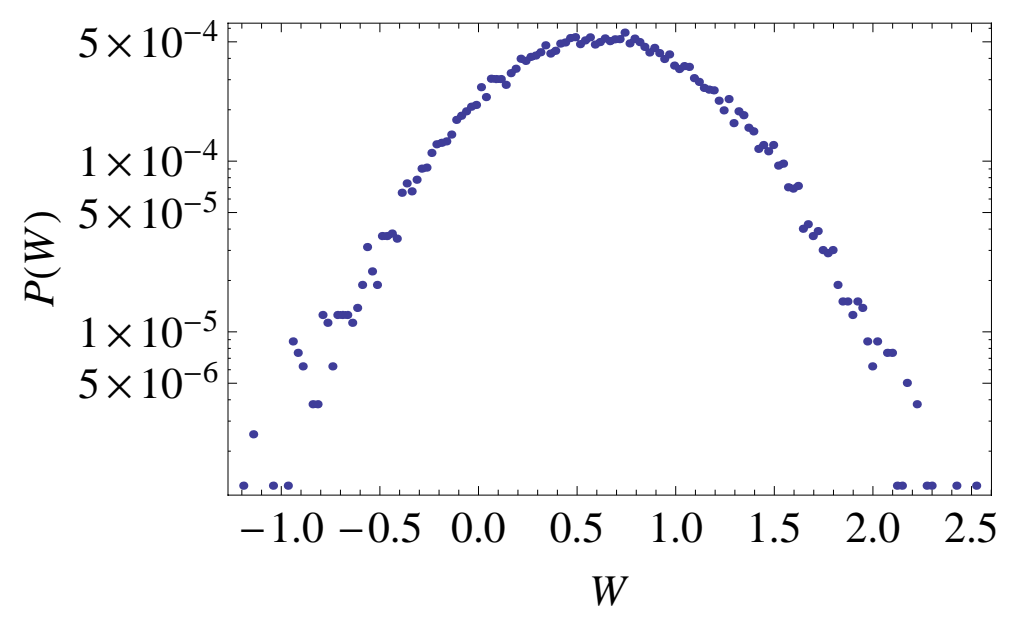

Figura 4.10: Distribuição de probabilidade para o trabalho realizado por um sistema externo, de acordo com o protocolo $L(t)$. 


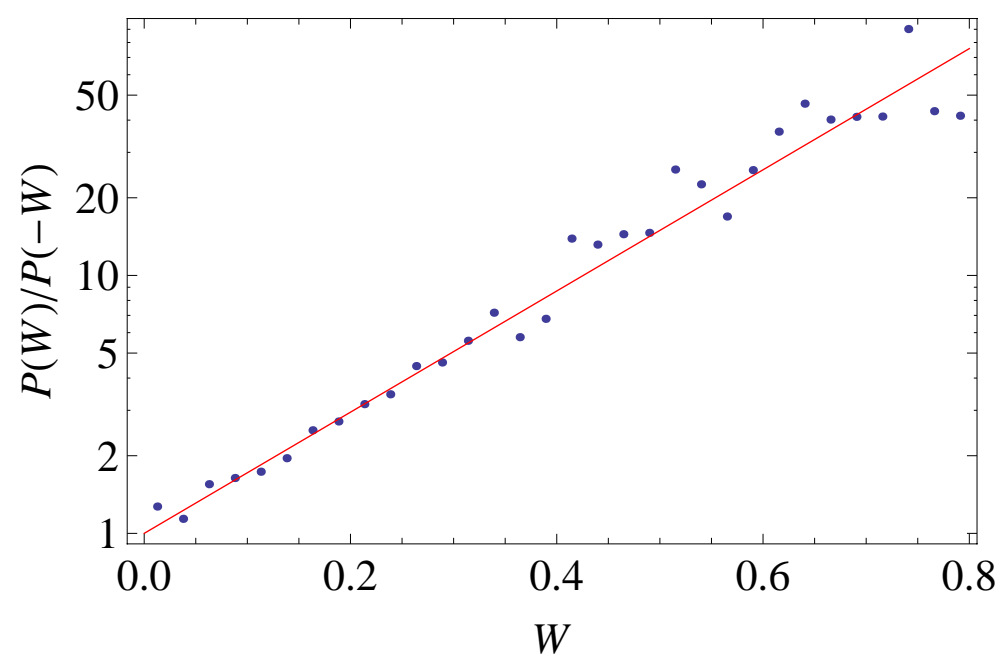

Figura 4.11: Relação de flutuação obtida para o trabalho realizado por um sistema externo, de acordo com o protocolo $L(t)$.

\subsection{3}

\section{Análise da entropia}

Na Figura (4.12), a evolução da entropia fora do equilíbrio $S(t)$ pode ser verificada. Podemos observar que o comportamento bimodal das oscilações contidas na predição teórica para o modelo linear, descritas anteriormente, também são apresentadas. No modelo não linear, a presença de um fraco acoplamento do potencial quártico $\left(k_{3} / k_{1}^{2} \ll 1\right)$ suscita uma série harmônica de picos sobre o espectro das oscilações da entropia. Isto pode ser verificado na Figura (4.13).

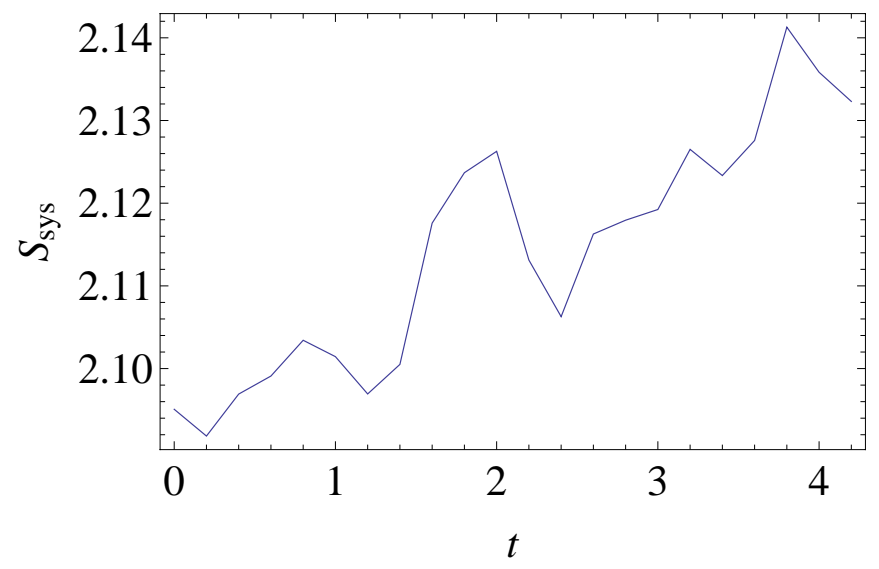

Figura 4.12: Evolução da entropia instantânea $S(t)$ para o modelo não linear. A produção da entropia fica caracterizada pela inclinação da curva. 


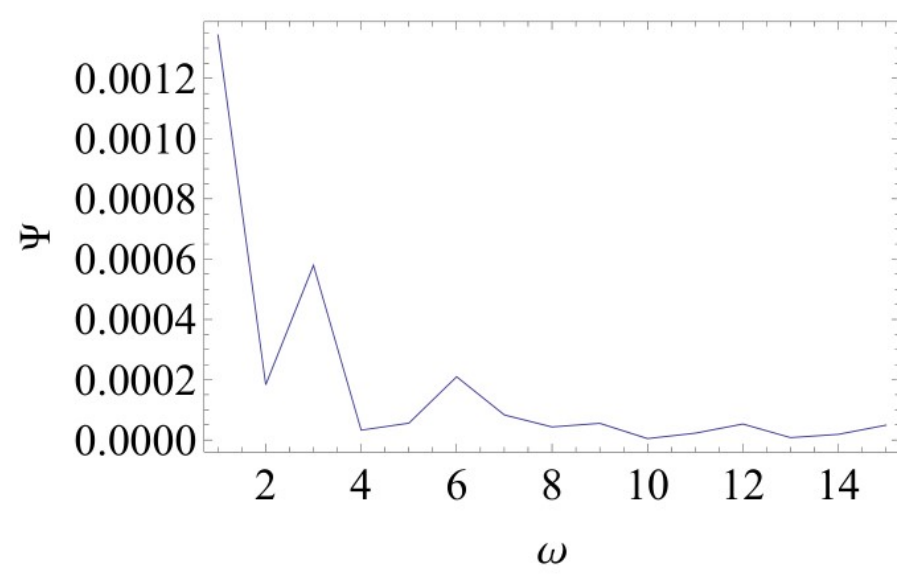

Figura 4.13: Análise espectral do comportamento de $S(t)$ para o modelo não linear.

\section{4}

\section{Estados de "almoço grátis"}

O último resultado apresentado neste trabalho é o chamado "almoço grátis", em alusão ao termo popularizado pelo economista e estatístico Milton Friedman (free lunch). No âmbito da estatística de sistemas fora do equilíbrio, representa os estados associados à energia tomada sem nenhum "custo" do reservatório. A definição ficará mais evidente com o exemplo que descreveremos na sequência.

Primeiramente, vamos relembrar uma importante relação derivada no segundo capítulo deste trabalho:

$$
\left\langle e^{-\beta W_{\text {diss }}}\right\rangle_{n-e q}=1
$$

Na qual $W_{\text {diss }}=W-\Delta F$ é o trabalho dissipado microscópico. Em virtude da igualdade na Eq. (4-78), esta relação é independente do protocolo realizado sobre o sistema. Então, podemos escolher um protocolo que tipicamente leva o sistema para muito longe dos estados de equilíbrio, produzindo valores numericamente grandes para o trabalho dissipado. De modo que:

$$
\beta\left\langle W_{\text {diss }}\right\rangle \gg 1 \Rightarrow 0<e^{-\beta W_{\text {diss }}} \ll 1 .
$$

A probabilidade de se obter a medida $e^{-\beta W_{\text {diss }}} \ll 1$ em um experimento desse tipo é muito alta (quase 1). Esta característica sugere um questionamento: como a IJ se mantém neste contexto? Isto acontece porque este procedimento suscita alguns casos muito improváveis, nos quais $W_{\text {diss }}<0 \Rightarrow$ $e^{-\beta W_{\text {diss }}}>1$. Estes estados são chamados de "almoço grátis" (AG) [88].

Podemos construir um caso simples de um AG ao analisarmos a expansão de um sistema formado por um gás ideal ( $N$ partículas), confinado por paredes termicamente condutoras, constantemente em contato com um reservatório 


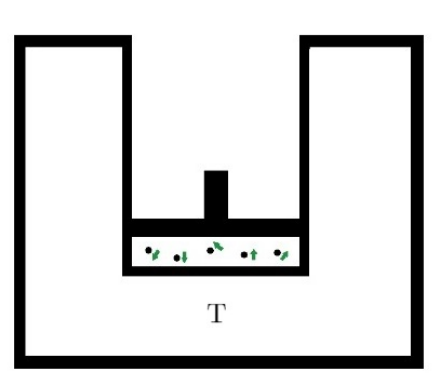

(a)

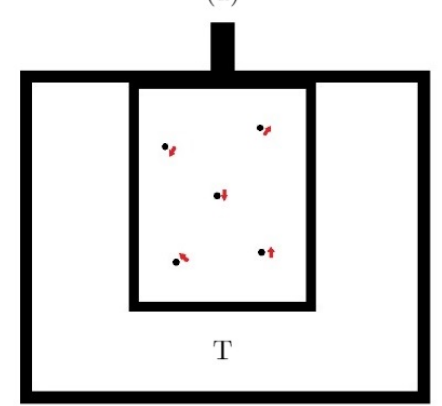

(d)

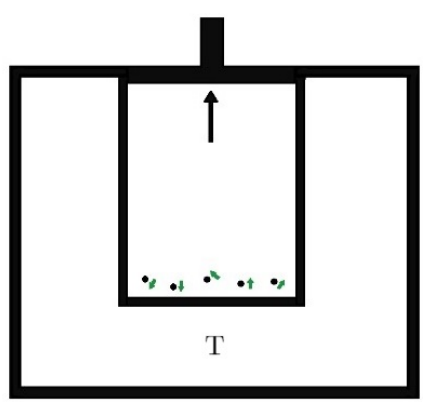

(b)

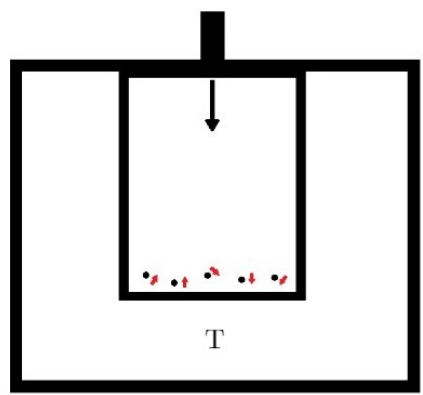

(e)

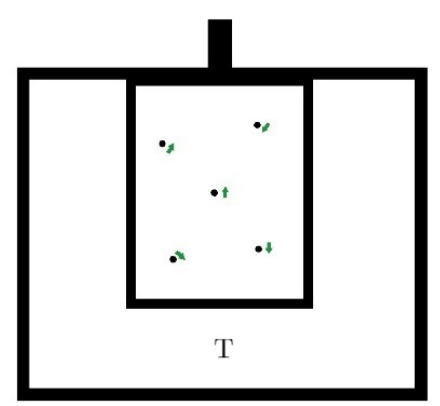

(c)

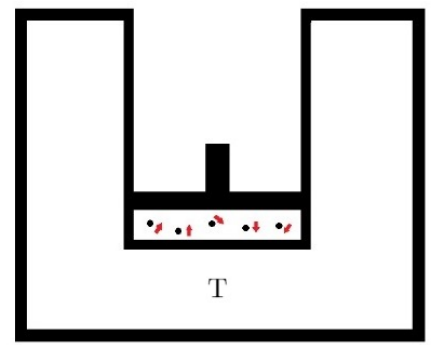

(f)

Figura 4.14: O processo de expansão está ilustrado em (a), (b) e (c) e o processo de compressão, em (d), (e) e (f). Os últimos descrevem a etapa em que surgem os estados de "almoço grátis".

térmico à temperatura $T=\beta^{-1}$, e um pistão isolado termicamente, controlado por um dado protocolo externo. Vamos chamar o processo de expansão deste sistema de $X$.

O gás está inicialmente $(t=0)$ com um volume $V_{i}=V_{0}$, e em um estado de equilíbrio macroscópico $M_{0}$. Escolhemos um microestado particular, $\mu_{0}=\left\{\mathbf{r}_{0}^{N}, \mathbf{p}_{0}^{N}\right\} \in M_{0}$, como a condição inicial. Note que $M_{0}$ está em um estado de equilíbrio macroscópico à volume $V_{0}$. Então, em $t=0$ o pistão é movido rapidamente, à velocidade $v_{p}$, da sua posição inicial até a sua posição final, em $t=\tau_{1}$ (mais rápido do que qualquer partícula do gás e, por esta razão, nenhuma das partículas do gás tem contato com o pistão). O volume inicial é alterado de acordo com a proporção $V\left(\tau_{1}\right)=V_{f}=100 V_{0}$, mas o pistão não realiza nenhum trabalho sobre o sistema.

Após a última etapa descrita, é concedido ao sistema um tempo $\tau_{2}$ que seja suficiente para que ele atinja o equilíbrio térmico com o reservatório (com $V_{f}$ constante), de modo que $\tau_{2} \gg 1 \gg \tau_{1}$. Em $t=\tau_{1}+\tau_{2}$, o sistema está no microestado $\mu_{f}=\left\{\mathbf{r}_{t=\tau_{1}+\tau_{2}}^{N}, \mathbf{p}_{t=\tau_{1}+\tau_{2}}^{N}\right\}=\left\{\mathbf{r}^{* N}, \mathbf{p}^{* N}\right\} \in M_{f}$, no qual $M_{f}$ é um estado de equilíbrio macroscópico, cujos valores da temperatura e volume são $\left(V=100 V_{0}, T\right)$.

Em uma segunda fase, o protocolo utilizado para o processo segue as seguintes etapas: 
- o gás está inicialmente em equilíbrio térmico $\left(100 V_{0}, T\right)$, no estado macroscópico $M_{f}$;

- de $t=0$ até $t=\tau_{2}$, o pistão é mantido na posição inicial;

- de $t=\tau_{2}$ até $t=\tau_{2}+\tau_{1}$, o pistão é movido rapidamente, à velocidade oposta $-v_{p}$, de tal modo que o volume final seja $V_{0}$.

O sistema não recebe nenhuma contribuição do trabalho na primeira fase, mas, em geral, uma quantidade enorme de trabalho deve ser feita sobre o sistema na segunda fase, a fim de comprimir violentamente o sistema $\left(100 V_{0} \rightarrow V_{0}\right.$. $)$. Então, o trabalho dissipado, $W_{\text {diss }}=W_{\text {ext }}-\left(F\left(V_{0}\right)-F\left(100 V_{0}\right)\right)$, também será muito grande.

Para a segunda fase, escolhemos um estado microscópico inicial $\mu_{i}^{\prime}=$ $\overline{\mu_{f}} \equiv\left\{\mathbf{r}^{* N},-\mathbf{p}^{* N}\right\}$, que está em equilíbrio térmico no começo do protocolo. Este microestado é obtido invertendo todas as velocidades das partículas do gás, e está no mesmo estado de equilíbrio macroscópico $M_{f}$ (devido ao princípio do balanço detalhado) [89]. Consequentemente, o gás irá percorrer a trajetória reversa, definida no segundo capítulo deste trabalho (Figura (2.7)), e espontaneamente evoluir na direção do estado final $\mu_{f}^{\prime}=\overline{\mu_{0}} \equiv\left\{\mathbf{r}_{0}^{N},-\mathbf{p}_{0}^{N}\right\}$. Devido à esta configuração para a trajetória reversa, durante a segunda fase o gás também não terá nenhum contato com o pistão, pois tanto as partículas do gás quanto o pistão estão retrocedendo as trajetórias da expansão $X$. Então, dada a condição inicial $\mu_{i}^{\prime}=\overline{\mu_{f}}$, o trabalho externo também será nulo na segunda fase, $W_{\text {ext }}=0$. O microestado final $\mu_{f}^{\prime}=\overline{\mu_{0}}$ está no estado de equilíbrio macroscópico $M_{0}$, cujos valores da temperatura e volume são $\left(V_{0}, T\right)$.

Finalmente, para este processo, o trabalho dissipado pelo sistema pode ser facilmente calculado:

$$
W_{F L, \text { disip }}=0-\Delta F=T \Delta S=-N T \ln \left(\frac{V}{V_{0}}\right)=-N T \ln 100,
$$

produzindo a expressão

$$
e^{-\beta W_{F L, d i s s i p}}=e^{N \ln 100}=100^{N} \gg 1,
$$

a qual revela uma grande contribuição ao valor esperado da quantidade $e^{-\beta W}$.

Os microestados similares a $\mu_{i}^{\prime}$ são extremamente raros, e a sua contribuição para as médias instantâneas pode ser pequena, mas o efeito sobre o comportamento da entropia pode ser considerável, devido à indução do decrescimento da entropia instantânea. 


\section{5}

\section{Conclusões e perspectivas}

A estrutura desta tese corresponde ao esforço de ser um trabalho autocontido, dentro dos conhecimentos de um graduado em Física. Com este objetivo, os dois capítulos iniciais foram escritos para servirem de base conceitual e descrição das técnicas necessárias para o desenvolvimento do trabalho original, que se dá no quarto capítulo.

Desse modo, acreditamos que no segundo capítulo conseguimos descrever a importância da Igualdade de Jarzinsky no âmbito dos Teoremas de Flutuação, demonstrando uma forma dessa relação ser obtida, e, também, mostramos alguns resultados interessantes neste contexto. Como já citado, existem outros diversos teoremas de flutuação, com diversas aplicações, mas acreditamos que, para o objetivo deste trabalho, a demonstração da IJ é o suficiente para aguçar a curiosidade do leitor.

No terceiro capítulo, apresentamos os desenvolvimentos que influenciaram na criação do trabalho original, contido no quarto capítulo. Descrevemos o modelo de evolução temporal pela equação de Langevin, assim como as técnicas utilizadas para calcular a distribuição de probabilidade das condições iniciais e os cumulantes do trabalho. Ainda, demonstramos o uso da IJ como calibre para a validade do modelo.

O quarto capítulo encerra os resultados originais desta tese. Analisamos os aspectos do modelo linear (harmônico) e não linear (potencial quártico) de um sistema massa-mola, sujeito a um protocolo de trabalho externo. Demonstramos a IJ para o modelo linear não markoviano proposto, e estudamos as decorrências do fluxo do calor, do trabalho e da entropia. Obtemos uma característica muito interessante neste cenário: partindo de uma distribuição de equilíbrio, independentemente da escolha do protocolo, nenhuma entropia é produzida no sistema de interesse para o caso linear.

Para o caso não linear, a análise numérica do comportamento da distribuição de probabilidade do calor injetado revela uma esperada assimetria, e obedece à proporcionalidade do respectivo teorema de flutuação. Do mesmo modo, a distribuição para o trabalho externo, realizado sobre o sistema de interesse, obedece à proporcionalidade do teorema de Crooks.

O comportamento da entropia, no caso não linear, apresenta um aspecto 
interessante: o núcleo de memória não markoviano atua de forma que tende a recuperar as informações perdidas, do sistema de interesse para o reservatório, durante a execução do protocolo.

O espectro da entropia, tanto o caso linear quanto o caso não linear, apresenta uma série harmônica de picos. A presença do termo não linear restaura a "normalidade" da evolução da entropia e sua produção durante a atuação do protocolo. Neste caso, observamos que a produção de entropia, no sistema de interesse, não é nula.

Como perspectiva para novos desenvolvimentos, no contexto deste trabalho, o cálculo analítico para a primeira ordem do caso não linear (em progresso) será útil para futuras comparações com os resultados numéricos obtidos. Ainda, a utilização de outras ferramentas para induzir um processo não-Markoviano (derivadas fracionárias, por exemplo), e a utilização de dimensões não inteiras para as equações que modelam o sistema, podem revelar novas características do comportamento das flutuações fora do equilíbrio descritas. 


\section{Referências bibliográficas}

[1] EVANS, D. J.; SEARLES, D. J.. The fluctuation theorem. Advances in Physics, 51 (7): 1529-1585, 2002.

[2] EINSTEIN, A.. On the theory of the brownian movement. Ann. Phys, 19 (4): 371-381, 1906.

[3] SMOLUCHOWSKI, M.. On the mean path of molecules of gas and its relationship to the theory of diffusion. Bullet Intern Acad Cracovie, p. 202-213, 1906.

[4] PERRIN, J.. Atoms (dl hammick, trans.). New York. Van Norstrand Company, 1913.

[5] MARCONI, U. M. B.; PUGLISI, A.; RONDONI, L. ; VULPIANI, A.. Fluctuation-dissipation: response theory in statistical physics. Physics reports, 461 (4-6): 111-195, 2008.

[6] BOCHKOV, G.; KUZOVLEV, Y. E.. General theory of thermal fluctuations in nonlinear systems. Zh. Eksp. Teor. Fiz, 72: 238-243, 1977.

[7] EVANS, D. J.; COHEN, E. G. D. ; MORRISS, G. P.. Probability of second law violations in shearing steady states. Physical review letters, 71 (15): 2401, 1993.

[8] JARZYNSKI, C.. Nonequilibrium equality for free energy differences. Physical Review Letters, 78 (14): 2690, 1997.

[9] JARZYNSKI, C.. Equalities and inequalities: Irreversibility and the second law of thermodynamics at the nanoscale. Annu. Rev. Condens. Matter Phys., 2 (1): 329-351, 2011.

[10] LAVERY, R.; LEBRUN, A.; ALLEMAND, J.-F.; BENSIMON, D. ; CROQUETTE, V.. Structure and mechanics of single biomolecules: experiment and simulation. Journal of Physics: Condensed Matter, 14 (14): R383, 2002.

[11] JAYNES, E. T.. Information theory and statistical mechanics. Physical review, 106 (4): 620, 1957. 
[12] KURCHAN, J.. Fluctuation theorem for stochastic dynamics. Journal of Physics A: Mathematical and General, 31 (16): 3719, 1998.

[13] SEKIMOTO, K.. Stochastic energetics, volumen 799. Springer, 2010.

[14] GALLAVOTTI, G.; COHEN, E. G. D.. Dynamical ensembles in nonequilibrium statistical mechanics. Physical review letters, 74 (14): 2694 , 1995.

[15] LEBOWITZ, J. L.; SPOHN, H.. A gallavotti-cohen-type symmetry in the large deviation functional for stochastic dynamics. Journal of Statistical Physics, 95 (1-2): 333-365, 1999.

[16] CROOKS, G. E.. Path-ensemble averages in systems driven far from equilibrium. Physical review E, 61 (3): 2361, 2000.

[17] BOCHKOV, G.; KUZOVLEV, Y. E.. Nonlinear fluctuation-dissipation relations and stochastic models in nonequilibrium thermodynamics: I. generalized fluctuation-dissipation theorem. Physica A: Statistical Mechanics and its Applications, 106 (3): 443-479, 1981.

[18] CROOKS, G. E.. Nonequilibrium measurements of free energy differences for microscopically reversible markovian systems. Journal of Statistical Physics, 90 (5-6): 1481-1487, 1998.

[19] ADIB, A. B.. Entropy and density of states from isoenergetic nonequilibrium processes. Physical Review E, 71 (5): 056128, 2005.

[20] JARZYNSKI, C.. Equalities and inequalities: irreversibility and the second law of thermodynamics at the nanoscale. In: TIME, p. 145172. Springer, 2013.

[21] CALLEN, H. B.. Thermodynamics and an introduction to thermostatistics, 1998.

[22] REX, A.; FINN, C.. Finn's Thermal Physics. CRC Press, 2017.

[23] CHENG, Y.-C.. Macroscopic and Statistical Thermodynamics: Expanded English. World Scientific Publishing Company, 2006.

[24] WEI, B.-B.; PLENIO, M. B.. Relations between dissipated work in non-equilibrium process and the family of rényi divergences. New Journal of Physics, 19 (2): 023002, 2017.

[25] SALINAS, S. R.. Introdução a física estatística vol. 09. Edusp, 1997. 
[26] HUANG, K.. Statistical mechanics. stme, p. 512, 1987.

[27] JARZYNSKI, C.. Nonequilibrium work theorem for a system strongly coupled to a thermal environment. Journal of Statistical Mechanics: Theory and Experiment, 2004 (09): P09005, 2004.

[28] ROGEL-SALAZAR, J.. Statistical mechanics, 3rd edn., by rk pathria and pd beale: Scope: textbook. level: postgraduate or advanced undergraduate, 2011.

[29] GOLDSTEIN, H.; POOLE, C. ; SAFKO, J.. Classical mechanics, 2002.

[30] JARZYNSKI, C.. Equilibrium free energies from nonequilibrium processes. arXiv preprint cond-mat/9802155, 1998.

[31] SEKIMOTO, K.. Langevin equation and thermodynamics. Progress of Theoretical Physics Supplement, 130: 17-27, 1998.

[32] KIRKWOOD, J. G.. Statistical mechanics of fluid mixtures. The Journal of Chemical Physics, 3 (5): 300-313, 1935.

[33] SCHURR, J. M.; FUJIMOTO, B. S.. Equalities for the nonequilibrium work transferred from an external potential to a molecular system. analysis of single-molecule extension experiments. The Journal of Physical Chemistry B, 107 (50): 14007-14019, 2003.

[34] CASELLA, G.; BERGER, R. L.. Statistical inference, volumen 2. Duxbury Pacific Grove, CA, 2002.

[35] LAGE, E.. Mecânica Avançada: EJS Lage, volumen 41. U. Porto Edições-Universidade do Porto, 2015.

[36] MURUGESHAN, R.; KIRUTHIGA, S.. Modern physics. S. Chand Publishing, 2017.

[37] IMPARATO, A.; PELITI, L.. Work distribution and path integrals in general mean-field systems. EPL (Europhysics Letters), 70 (6): 740, 2005.

[38] SEIFERT, U.. Entropy production along a stochastic trajectory and an integral fluctuation theorem. Physical review letters, 95 (4): 040602, 2005.

[39] CUENDET, M. A.. Statistical mechanical derivation of jarzynski's identity for thermostated non-hamiltonian dynamics. Physical review letters, 96 (12): 120602, 2006. 
[40] CHELLI, R.; MARSILI, S.; BARDUCCI, A. ; PROCACCI, P.. Generalization of the jarzynski and crooks nonequilibrium work theorems in molecular dynamics simulations. Physical Review E, 75 (5): 050101, 2007.

[41] GE, H.; JIANG, D.. Generalized jarzynski's equality of multidimensional inhomogeneous diffusion processes. J Stat Phys, 131: 675-689, 2008.

[42] LIPHARDT, J.; DUMONT, S.; SMITH, S. B.; TINOCO, I. ; BUSTAMANTE, C.. Equilibrium information from nonequilibrium measurements in an experimental test of jarzynski's equality. Science, 296 (5574): 1832-1835, 2002.

[43] BLICKLE, V.; SPECK, T.; HELDEN, L.; SEIFERT, U. ; BECHINGER, C.. Thermodynamics of a colloidal particle in a time-dependent nonharmonic potential. Physical review letters, 96 (7): 070603, 2006.

[44] DOUARChE, F.; CILIBERTO, S.; Petrosyan, A. ; RABBiosi, I.. An experimental test of the jarzynski equality in a mechanical experiment. EPL (Europhysics Letters), 70 (5): 593, 2005.

[45] MOSSA, A.; DE LORENZO, S.; HUGUET, J. M. ; RITORT, F.. Measurement of work in single-molecule pulling experiments. The Journal of chemical physics, 130 (23): 234116, 2009.

[46] JARZYNSKIA, C.. Nonequilibrium work relations: foundations and applications. The European Physical Journal B, 64 (3-4): 331-340, 2008.

[47] RONDONI, L.; MEJÍA-MONASTERIO, C.. Fluctuations in nonequilibrium statistical mechanics: models, mathematical theory, physical mechanisms. Nonlinearity, 20 (10): R1, 2007.

[48] SEIFERT, U.. Stochastic thermodynamics: principles and perspectives. The European Physical Journal B, 64 (3-4): 423-431, 2008.

[49] CLEUREN, B.; VAN DEN BROECK, C. ; KAWAI, R.. Fluctuation and dissipation of work in a joule experiment. Physical review letters, 96 (5): 050601, 2006.

[50] CROOKS, G. E.. Entropy production fluctuation theorem and the nonequilibrium work relation for free energy differences. Physical Review E, 60 (3): 2721, 1999. 
[51] COLLIN, D.; RITORT, F.; JARZYNSKI, C.; SMITH, S. B.; TINOCO, I. ; BUSTAMANTE, C.. Verification of the crooks fluctuation theorem and recovery of rna folding free energies. Nature, 437 (7056): 231234, 2005.

[52] DOUARCHE, F.; JOUBAUD, S.; GARNIER, N. B.; PETROSYAN, A. ; CILIBERTO, S.. Work fluctuation theorems for harmonic oscillators. Physical review letters, 97 (14): 140603, 2006.

[53] TOLMAN, R. C.. The principle of microscopic reversibility. Proceedings of the National Academy of Sciences of the United States of America, 11 (7): 436, 1925.

[54] QIAN, H.. Relative entropy: Free energy associated with equilibrium fluctuations and nonequilibrium deviations. Physical Review E, 63 (4): 042103, 2001.

[55] COVER, T. M.; THOMAS, J. A.. Elements of information theory. John Wiley \& Sons, 2012.

[56] JARZYNSKI, C.. Rare events and the convergence of exponentially averaged work values. Physical Review E, 73 (4): 046105, 2006.

[57] UHLENBECK, G. E.; FORD, G. ; MONTROLL, E. W.. Lectures in statistical mechanics; with an appendix on quantum statistics of interacting particles. Technical report, American Mathematical Society Providence RI, 1961.

[58] KAWAI, R.; PARRONDO, J. M. ; VAN DEN BROECK, C.. Dissipation: The phase-space perspective. Physical review letters, 98 (8): 080602, 2007.

[59] PARK, S.; SCHULTEN, K.. Calculating potentials of mean force from steered molecular dynamics simulations. The Journal of chemical physics, 120 (13): 5946-5961, 2004.

[60] RUELLE, D.. Statistical mechanics: Rigorous results. World Scientific, 1999.

[61] MORGADO, W. A.; QUEIRÓS, S. M. D.. Thermostatistics of small nonlinear systems: Gaussian thermal bath. Physical Review E, 90 (2): 022110, 2014.

[62] J EVANS, D.; P MORRISS, G.. Statistical mechanics of nonequilbrium liquids. ANU Press, 2007. 
[63] LANDO, S. K.. Lectures on generating functions, volumen 23. American Mathematical Soc., 2003.

[64] SOARES-PINTO, D. O.; MORGADO, W. A. M.. Brownian dynamics, time-averaging and colored noise. Physica A: Statistical Mechanics and its Applications, 365 (2): 289-299, 2006.

[65] MORGADO, W.; SOARES-PINTO, D.. Exact nonequilibrium work generating function for a small classical system. Physical Review E, 82 (2): 021112, 2010.

[66] VAN KAMPEN, N. G.. Stochastic processes in physics and chemistry, volumen 1. Elsevier, 1992.

[67] BIRKHOFF, G. D.. Proof of the ergodic theorem. Proceedings of the National Academy of Sciences, 17 (12): 656-660, 1931.

[68] SCHIFF, J. L.. The Laplace transform: theory and applications. Springer Science \& Business Media, 2013.

[69] CALLEN, H. B.; WELTON, T. A.. Irreversibility and generalized noise. Physical Review, 83 (1): 34, 1951.

[70] BUDINI, A. A.; CÁCERES, M. O.. Functional characterization of generalized langevin equations. Journal of Physics $A$ : Mathematical and General, 37 (23): 5959, 2004.

[71] VIGNAT, C.. A generalized isserlis theorem for location mixtures of gaussian random vectors. Statistics \& probability letters, 82 (1): 67-71, 2012.

[72] MORGADO, W.; SOARES-PINTO, D.. Exact time-averaged thermal conductance for small systems: Comparison between direct calculation and green-kubo formalism. Physical Review E, 79 (5): 051116 , 2009.

[73] WYLD, H.. Methods for physics, 1993.

[74] ZILL, D. G.; CULLEN, M.. Differential equations. Brooks/Cole, Cengage Learning., 2009.

[75] DUFFY, D. G.. Green's functions with applications. CRC Press, 2015.

[76] KUTVONEN, A.; ALA-NISSILA, T. ; PEKOLA, J.. Entropy production in a non-markovian environment. Physical Review E, 92 (1): 012107, 2015. 
[77] SANJUÁN, M. A.. Nonequilibrium statistical physics: a modern perspective. Contemporary Physics, 59: 316-317, 2018.

[78] HÄNGGI, P.. Generalized langevin equations: A useful tool for the perplexed modeller of nonequilibrium fluctuations? In: STOCHASTIC DYNAMICS, p. 15-22. Springer, 1997.

[79] HOROWITZ, J.; JARZYNSKI, C.. Comparison of work fluctuation relations. Journal of Statistical Mechanics: Theory and Experiment, 2007 (11): P11002, 2007.

[80] DHAR, A.. Heat transport in low-dimensional systems. Advances in Physics, 57 (5): 457-537, 2008.

[81] ARFKEN, G. B.; WEBER, H. J.. Mathematical methods for physicists, 1999.

[82] LIMA, E. L.; OTHERS. A equação do terceiro grau. Matemática Universitária, 5: 9-23, 1987.

[83] HAMEDANI, G.; TATA, M.. On the determination of the bivariate normal distribution from distributions of linear combinations of the variables. The American Mathematical Monthly, 82 (9): 913-915, 1975.

[84] VAN KAMPEN, N.; OPPENHEIM, I.. Brownian motion as a problem of eliminating fast variables. Physica A: Statistical Mechanics and its Applications, 138 (1-2): 231-248, 1986.

[85] KANAZAWA, K.; SAGAWA, T. ; HAYAKAWA, H.. Heat conduction induced by non-gaussian athermal fluctuations. Physical Review E, 87 (5): 052124, 2013.

[86] MORGADO, W. A.; QUEIRÓS, S. M. D.. Thermostatistics of small nonlinear systems: Poissonian athermal bath. Physical Review E, 93 (1): 012121, 2016.

[87] JARZYNSKI, C.; WÓJCIK, D. K.. Classical and quantum fluctuation theorems for heat exchange. Physical review letters, 92 (23): 230602, 2004.

[88] PELlegrinI, F.; PANIZON, E.; SANTORO, G. E. ; TOSATTI, E.. Thermally assisted lubricity and negative work tails in sliding friction. Physical Review B, 99 (7): 075428, 2019. 
[89] DE GROOT, S. R.; MAZUR, P.. Non-equilibrium thermodynamics. Courier Corporation, 2013.

[90] REITH, D.; PÜTZ, M. ; MÜLLER-PLATHE, F.. Deriving effective mesoscale potentials from atomistic simulations. Journal of computational chemistry, 24 (13): 1624-1636, 2003. 


\section{A.1}

\section{Considerações sobre a hamiltoniana de interação}

No âmbito da dinâmica molecular, o potencial de força média é uma aproximação das interações das $N$ partículas, de um fluido, atuando sobre uma partícula específica, mantida fixa em relação às partículas remanescentes que compõem o sistema [32]. Geralmente, este potencial é utilizado como linha condutora para otimizar o potencial efetivo entre as partículas do sistema [90]. No contexto deste trabalho, vamos escrever o potencial de força média na forma:

$$
\mathcal{H}^{\dagger}(\boldsymbol{x} ; \lambda) \equiv \mathcal{H}(\boldsymbol{x} ; \lambda)-\beta^{-1} \ln \left(\frac{\int d y \exp \left[-\beta\left\{\mathcal{H}_{\text {res }}(\boldsymbol{y})+h_{\text {int }}(\boldsymbol{x}, \boldsymbol{y})\right\}\right]}{\int d y \exp \left[-\beta \mathcal{H}_{\text {res }}(\boldsymbol{y})\right]}\right) .
$$

Assim, a função de partição do sistema de interesse e a função de partição do sistema de interesse e reservatório térmico combinados, são, respectivamente:

$$
Z_{\lambda}=\int d \boldsymbol{x} \exp \left[-\beta \mathcal{H}^{\dagger}(\boldsymbol{x} ; \lambda)\right]
$$

$\mathrm{e}$

$$
Y_{\lambda}=\int d \boldsymbol{x} d \boldsymbol{y} \exp \left[-\beta\left\{\mathcal{H}(\boldsymbol{x} ; \lambda)+\mathcal{H}_{\text {res }}(\boldsymbol{y})+h_{\text {int }}(\boldsymbol{x}, \boldsymbol{y})\right\}\right] .
$$

Daí, podemos escrever:

$$
Y_{\lambda}=Z_{\lambda} \int d y \exp \left[-\beta \mathcal{H}_{\text {res }}(\boldsymbol{y})\right] .
$$

Portanto, sem recorrer à suposição de uma fraca interação da Hamiltoniana $h_{\text {int }}(\boldsymbol{x}, \boldsymbol{y})$, temos o mesmo resultado da Eq. (2-27), a saber:

$$
\frac{Y_{B}}{Y_{A}}=\frac{Z_{B}}{Z_{A}} .
$$


B

\section{B.1}

Derivação da expressão de $\tilde{x}(s)$ e $\tilde{v}(s)$

Para o modelo idealizado, temos a seguinte equação:

$$
m v(t)=m v_{0}-\gamma \int_{0}^{t} v\left(t^{\prime}\right) d t^{\prime}-k x_{0} t-k \int_{0}^{t} \int_{0}^{t^{\prime}} v\left(t^{\prime \prime}\right) d t^{\prime \prime} d t^{\prime}+\int_{0}^{t} \eta\left(t^{\prime}\right) d t^{\prime}
$$

Aplicando a transformada de Laplace sobre a Eq. (B-1), obtemos:

$$
m \tilde{v}(s)=\frac{m v_{0}}{s}-\frac{\gamma \tilde{v}(s)}{s}-\frac{k\left(x_{0}+\tilde{v}(s)\right)}{s^{2}}+\frac{\tilde{\eta}(s)}{m s},
$$

na qual $\tilde{f}(s)=\int_{0}^{\infty} d t e^{-s t} f(t), x_{0}=x(0)$ e $v_{0}=v(0)$.

Tomando o termo $\tilde{v}(s)$ em evidência, separando a parte das condições iniciais da parte do ruído térmico, temos a relação:

$$
\tilde{v}(s)=\frac{s v_{0}-w_{0}^{2} x_{0}}{s(s+\beta)+w_{0}^{2}}+\frac{m^{-1} s}{s(s+\beta)+w_{0}^{2}} \tilde{\eta}(s),
$$

na qual $w_{0}=k / m$ e $\beta=\gamma / m$. A relação para $\tilde{x}(s)$ é derivada diretamente utilizando a relação $x(t)=x_{0}+\int_{0}^{t} v\left(t^{\prime}\right) d t^{\prime}$.

\section{B.2}

\section{A distribuição de equilíbrio para a posição $x$}

Podemos expressar a evolução temporal da distribuição de probabilidade para a posição em função do valor esperado da transformada de Laplace de $x(t)$. Os procedimentos são os seguintes: 


$$
\begin{aligned}
p(x, t)= & \langle\delta(x-x(t))\rangle_{\xi} \\
= & \int_{-\infty}^{\infty} \frac{d Q}{2 \pi}\left\langle e^{i Q(x-x(t))}\right\rangle_{\xi} \\
= & \int_{-\infty}^{\infty} \frac{d Q}{2 \pi} e^{i Q x}\left\langle e^{-i Q x(t)}\right\rangle_{\xi} \\
= & \sum_{m=0}^{\infty} \frac{1}{m !} \int_{-\infty}^{\infty} \frac{d Q}{2 \pi}(-i Q)^{m} e^{i Q x}\left\langle x^{m}(t)\right\rangle_{\xi} \\
= & \sum_{m=0}^{\infty} \frac{1}{m !} \int_{-\infty}^{\infty} \frac{d Q}{2 \pi}(-i Q)^{m} e^{i Q x} \int_{0}^{\infty} d t_{1} \delta\left(t-t_{1}\right) \ldots \int_{0}^{\infty} d t_{m} \delta\left(t-t_{m}\right)\left\langle x\left(t_{1}\right) \ldots x\left(t_{m}\right)\right\rangle_{\xi} \\
= & \lim _{\epsilon \rightarrow 0} \sum_{m=0}^{\infty} \frac{1}{m !} \int_{-\infty}^{\infty} \frac{d Q}{2 \pi}(-i Q)^{m} e^{i Q x} \times \\
& \times \int_{0}^{\infty} d t_{1} \int_{-\infty}^{\infty} \frac{d q_{1}}{2 \pi} e^{\left(i q_{1}+\epsilon\right)\left(t-t_{1}\right)} \ldots \int_{0}^{\infty} d t_{m} \int_{-\infty}^{\infty} \frac{d q_{m}}{2 \pi} e^{\left(i q_{m}+\epsilon\right)\left(t-t_{m}\right)}\left\langle x\left(t_{1}\right) \ldots x\left(t_{m}\right)\right\rangle_{\xi} \\
= & \lim _{\epsilon \rightarrow 0} \sum_{m=0}^{\infty} \frac{1}{m !} \int_{-\infty}^{\infty} \frac{d Q}{2 \pi}(-i Q)^{m} e^{i Q x} \times \\
& \times \int_{-\infty}^{\infty} \frac{d q_{1}}{2 \pi} e^{\left(i q_{1}+\epsilon\right)(t)} \ldots \int_{-\infty}^{\infty} \frac{d q_{m}}{2 \pi} e^{\left(i q_{m}+\epsilon\right)(t)}\left\langle\tilde{x}\left(i q_{1}+\epsilon\right) \ldots \tilde{x}\left(i q_{m}+\epsilon\right)\right\rangle_{\xi} \\
= & \lim _{\epsilon \rightarrow 0} \sum_{m=0}^{\infty} \frac{1}{m !} \int_{-\infty}^{\infty} \frac{d Q}{2 \pi}(-i Q)^{m} e^{i Q x} \int_{-\infty}^{\infty} \prod_{i=1}^{m} \frac{d q_{i}}{2 \pi} e^{\sum_{j=1}^{m}\left(i q_{j}+\epsilon\right) t}\left\langle\prod_{i=1}^{m} \tilde{x}\left(i q_{i}+\epsilon\right)\right\rangle_{\xi} .
\end{aligned}
$$

\section{B.3}

\section{Cálculo da contribuição de $B(s) B(s)$}

Para evitar algebrismos desnecessários para esta demonstração, vamos calcular o resíduo desta contribuição com o ruído branco. Utilizando o ruído colorido, os cálculos são similares.

$$
\begin{aligned}
& \int_{-\infty}^{\infty} \int_{-\infty}^{\infty} \frac{d q_{i}}{2 \pi} \frac{d q_{j}}{2 \pi} \frac{z}{z-i\left(q_{i}+q_{j}+\bullet\right)} B\left(i q_{i}+\epsilon\right) B\left(i q_{j}+\epsilon\right)\left\langle\eta\left(i q_{i}+\epsilon\right) \eta\left(i q_{j}+\epsilon\right)\right\rangle= \\
= & \int_{-\infty}^{\infty} \int_{-\infty}^{\infty} \frac{d q_{i}}{2 \pi} \frac{d q_{j}}{2 \pi} \frac{z}{z-i\left(q_{i}+q_{j}+\square\right)} \frac{m^{-2}}{\left(q_{i}+i \kappa_{+}\right)\left(q_{i}+i \kappa_{-}\right)\left(q_{j}+i \kappa_{+}\right)\left(q_{j}+i \kappa_{-}\right)} \frac{D}{i\left(q_{i}+q_{j}\right)+2 \epsilon} \\
= & \frac{z}{z-i(0+\bullet)} \int_{-\infty}^{\infty} \frac{d q_{j}}{2 \pi} \frac{m^{-2} D}{\left(q_{j}-i \kappa_{+}\right)\left(q_{j}-i \kappa_{-}\right)\left(q_{j}+i \kappa_{+}\right)\left(q_{j}+i \kappa_{-}\right)} \\
= & \frac{D}{2 \gamma k} \frac{z}{z-i(0+\bullet)} .
\end{aligned}
$$




\section{B.4}

\section{Modelo não linear (correção até a ordem $\mathcal{O}\left(k_{3}^{1}\right)$ )}

Devido à forma da força proporcional a $x^{3}(t)$, o modelo descrito na Eq. (4-2) pode ser entendido como uma equação de recorrência de múltiplas ordens de $k_{3}$. Para isso, a correção de primeira ordem da função de distribuição de probalidade para o caso não linear deve ser utilizada. Assim, temos a seguinte forma para a correção da distribuição para a primeira ordem de $k_{3}$ :

$$
\begin{aligned}
p_{0}(x) & +k_{3} p_{1}(x) \approx \lim _{t \rightarrow \infty} \lim _{\epsilon \rightarrow 0} \sum_{m=0}^{\infty} \frac{1}{(2 m) !} \int_{-\infty}^{\infty} \frac{d Q}{2 \pi}(-i Q)^{2 m} e^{i Q x} \int_{-\infty}^{\infty} \prod_{i=1}^{2 m} \frac{d q_{i}}{2 \pi} e^{\sum_{j=1}^{2 m}\left(i q_{j}+\epsilon\right) t} \times \\
& \times \frac{1}{\prod_{i=1}^{2 m} R\left(i q_{i}+\epsilon\right)}\left\langle\prod_{i=1}^{2 m} \tilde{\xi}\left(i q_{i}+\epsilon\right)\right\rangle_{\xi} \\
& -\lim _{t \rightarrow \infty} \lim _{\epsilon \rightarrow 0} \lim _{\alpha \rightarrow 0^{+}} \sum_{m=0}^{\infty} \frac{1}{(2 m) !} \int_{-\infty}^{\infty} \frac{d Q}{2 \pi}(-i Q)^{2 m} e^{i Q x} \int_{-\infty}^{\infty} \prod_{i=1}^{2 m} \frac{d q_{i}}{2 \pi} e^{\sum_{j=1}^{2 m}\left(i q_{j}+\epsilon\right) t} \times \\
& \times \frac{1}{\prod_{i=1}^{2 m} R\left(i q_{i}+\epsilon\right)} \frac{k_{3}(2 m)}{R\left(i q_{-1}+\alpha\right) R\left(i q_{-2}+\alpha\right) R\left(i q_{-3}+\alpha\right)} \times \\
& \times \int_{-\infty}^{+\infty} \frac{d q_{-1}}{2 \pi} \frac{d q_{-2}}{2 \pi} \frac{d q_{-3}}{2 \pi} \frac{\left\langle\tilde{\xi}\left(i q_{-1}+\alpha\right) \tilde{\xi}\left(i q_{-2}+\alpha\right) \tilde{\xi}\left(i q_{-3}+\alpha\right) \prod_{i=2}^{2 m} \tilde{\xi}\left(i q_{i}+\epsilon\right)\right\rangle_{\xi}}{\left(i q_{1}+\epsilon\right)-\left(i q_{-1}+i q_{-2}+i q_{-3}+3 \alpha\right)}
\end{aligned}
$$




\section{B.5}

\section{Expressão dos termos $A_{i}$ do segundo cumulante}

$$
\begin{aligned}
& A_{1}=\left(\mathrm{e}^{-2 \frac{\theta}{\lambda}}-2 \mathrm{e}^{\frac{\left(\kappa_{1} \lambda-1\right) \theta}{\lambda}}+1\right) T L_{0}^{2}{k_{2}}^{2} m^{-2} \tau^{-2} \kappa_{1}{ }^{-1} \times \\
& \times\left(\kappa_{1}^{2} \lambda^{2}-1\right)^{-1}\left(\kappa_{1}^{2}-\kappa_{2}^{2}\right)^{-1}\left(\kappa_{1}^{2}-\kappa_{3}^{2}\right)^{-1}- \\
& -\left(\mathrm{e}^{-2 \frac{\theta}{\lambda}}-2 \mathrm{e}^{\frac{\left(\kappa_{2} \lambda-1\right) \theta}{\lambda}}+1\right) T L_{0}^{2}{k_{2}}^{2} m^{-2} \tau^{-2} \kappa_{2}{ }^{-1} \\
& \times\left(\kappa_{2}^{2} \lambda^{2}-1\right)^{-1}\left(\kappa_{1}^{2}-\kappa_{2}^{2}\right)^{-1}\left(\kappa_{2}^{2}-\kappa_{3}^{2}\right)^{-1}+ \\
& +\left(\mathrm{e}^{-2 \frac{\theta}{\lambda}}-2 \mathrm{e}^{\frac{\left(\kappa_{3} \lambda-1\right) \theta}{\lambda}}+1\right) T L_{0}{ }^{2}{k_{2}}^{2} m^{-2} \tau^{-2} \kappa_{3}{ }^{-1} \times \\
& \times\left(\kappa_{3}^{2} \lambda^{2}-1\right)^{-1}\left(\kappa_{1}^{2}-\kappa_{3}^{2}\right)^{-1}\left(\kappa_{2}^{2}-\kappa_{3}^{2}\right)^{-1}- \\
& \text { - }\left(\mathrm{e}^{-2 \frac{\theta}{\lambda}}-1\right) T L_{0}{ }^{2} k_{2}{ }^{2} \lambda^{5} m^{-2} \tau^{-2}\left(\kappa_{1}{ }^{2} \lambda^{2}-1\right)^{-1}\left(\kappa_{2}{ }^{2} \lambda^{2}-1\right)^{-1}\left(\kappa_{3}{ }^{2} \lambda^{2}-1\right)^{-1}+ \\
& +k_{2}^{2} L_{0}^{2} T\left(\mathrm{e}^{\frac{\left(\kappa_{1} \lambda-1\right) \theta}{\lambda}}-1\right)^{2}\left(1+\tau \kappa_{1}\right) m^{-2} \tau^{-2} \\
& \times\left(\kappa_{1} \lambda-1\right)^{-2}\left(\kappa_{1}-\kappa_{2}\right)^{-2}\left(\kappa_{1}-\kappa_{3}\right)^{-2} \kappa_{1}^{-1}- \\
& -2 k_{2}^{2} L_{0}^{2} T\left(\mathrm{e}^{\frac{\left(\kappa_{2} \lambda-1\right) \theta}{\lambda}}-1\right)\left(\mathrm{e}^{\frac{\left(\kappa_{1} \lambda-1\right) \theta}{\lambda}}-1\right) \times \\
& \times \frac{\left(2+\tau \kappa_{2}+\tau \kappa_{1}\right)}{m^{2} \tau^{2}\left(\kappa_{2} \lambda-1\right)\left(\kappa_{1} \lambda-1\right)\left(\kappa_{2}-\kappa_{3}\right)\left(\kappa_{1}-\kappa_{2}\right)\left(\kappa_{1}-\kappa_{3}\right)\left(\kappa_{1}+\kappa_{2}\right)}+ \\
& +2 k_{2}^{2} L_{0}^{2} T\left(\mathrm{e}^{\frac{\left(\kappa_{3} \lambda-1\right) \theta}{\lambda}}-1\right)\left(\mathrm{e}^{\frac{\left(\kappa_{1} \lambda-1\right) \theta}{\lambda}}-1\right) \times \\
& \times \frac{\left(2+\tau \kappa_{3}+\tau \kappa_{1}\right)}{m^{2} \tau^{2}\left(\kappa_{3} \lambda-1\right)\left(\kappa_{1} \lambda-1\right)\left(\kappa_{2}-\kappa_{3}\right)\left(\kappa_{1}-\kappa_{2}\right)\left(\kappa_{1}-\kappa_{3}\right)^{2}\left(\kappa_{1}+\kappa_{3}\right)}+ \\
& +k_{2}{ }^{2} L_{0}{ }^{2} T\left(\mathrm{e}^{\frac{\left(\kappa_{2} \lambda-1\right) \theta}{\lambda}}-1\right)^{2}\left(1+\tau \kappa_{2}\right) m^{-2} \tau^{-2} \times \\
& \times\left(\kappa_{2} \lambda-1\right)^{-2}\left(\kappa_{1}-\kappa_{2}\right)^{-2}\left(\kappa_{2}-\kappa_{3}\right)^{-2} \kappa_{2}{ }^{-1}- \\
& -2 k_{2}^{2} L_{0}^{2} T\left(\mathrm{e}^{\frac{\left(\kappa_{3} \lambda-1\right) \theta}{\lambda}}-1\right)\left(\mathrm{e}^{\frac{\left(\kappa_{2} \lambda-1\right) \theta}{\lambda}}-1\right)\left(2+\tau \kappa_{3}+\tau \kappa_{2}\right) m^{-2} \tau^{-2} \times \\
& \times\left(\kappa_{3} \lambda-1\right)^{-1}\left(\kappa_{2} \lambda-1\right)^{-1}\left(\kappa_{1}-\kappa_{3}\right)^{-1}\left(\kappa_{1}-\kappa_{2}\right)^{-1}\left(\kappa_{2}-\kappa_{3}\right)^{-2}\left(\kappa_{2}+\kappa_{3}\right)^{-1}+ \\
& +k_{2}^{2} L_{0}^{2} T\left(\mathrm{e}^{\frac{\left(\kappa_{3} \lambda-1\right) \theta}{\lambda}}-1\right)^{2}\left(1+\tau \kappa_{3}\right) m^{-2} \tau^{-2}\left(\kappa_{3} \lambda-1\right)^{-2}\left(\kappa_{1}-\kappa_{3}\right)^{-2} \times \\
& \times\left(\kappa_{2}-\kappa_{3}\right)^{-2} \kappa_{3}^{-1} \\
& A_{2}=k_{2}^{2} L_{0}^{2} T\left(\mathrm{e}^{-\frac{\theta}{\lambda}}-1\right)^{2}\left(k_{1}+k_{2}\right)^{-1} \\
& A_{3}+A_{4}=2 k_{2}{ }^{2} L_{0}{ }^{2} T\left(\mathrm{e}^{\frac{\left(\kappa_{1} \lambda-1\right) \theta}{\lambda}}-1\right)\left(\mathrm{e}^{-\frac{\theta}{\lambda}}-1\right)\left(1+\tau \kappa_{1}\right) m^{-1} \tau^{-1} \times \\
& \times\left(\kappa_{1} \lambda-1\right)^{-1}\left(\kappa_{1}-\kappa_{2}\right)^{-1}\left(\kappa_{1}-\kappa_{3}\right)^{-1} \kappa_{1}^{-1}- \\
& -2 k_{2}{ }^{2} L_{0}{ }^{2} T\left(\mathrm{e}^{\frac{\left(\kappa_{2} \lambda-1\right) \theta}{\lambda}}-1\right)\left(\mathrm{e}^{-\frac{\theta}{\lambda}}-1\right)\left(1+\tau \kappa_{2}\right) m^{-1} \tau^{-1} \times \\
& \times\left(\kappa_{2} \lambda-1\right)^{-1}\left(\kappa_{1}-\kappa_{2}\right)^{-1}\left(\kappa_{2}-\kappa_{3}\right)^{-1} \kappa_{2}^{-1}+ \\
& +2 k_{2}{ }^{2} L_{0}{ }^{2} T\left(\mathrm{e}^{\frac{\left(\kappa_{3} \lambda-1\right) \theta}{\lambda}}-1\right)\left(\mathrm{e}^{-\frac{\theta}{\lambda}}-1\right)\left(1+\tau \kappa_{3}\right) m^{-1} \tau^{-1} \times \\
& \times\left(\kappa_{3} \lambda-1\right)^{-1}\left(\kappa_{1}-\kappa_{3}\right)^{-1}\left(\kappa_{2}-\kappa_{3}\right)^{-1} \kappa_{3}{ }^{-1}+ \\
& +2 k_{2}{ }^{2} L_{0}{ }^{2} T\left(\mathrm{e}^{-\frac{\theta}{\lambda}}-1\right)^{2} m^{-1} \tau^{-1} \kappa_{1}{ }^{-1} \kappa_{2}{ }^{-1} \kappa_{3}{ }^{-1}
\end{aligned}
$$




$$
\begin{aligned}
& A_{5}=2 k_{2}^{2} L_{0}^{2} T\left(\mathrm{e}^{\frac{\left(\kappa_{1} \lambda-1\right) \theta}{\lambda}}-1\right)\left(\mathrm{e}^{-\frac{\theta}{\lambda}}-1\right)\left(1+\tau \kappa_{1}\right)\left(k_{1}+k_{2}\right) m^{-2} \tau^{-2} \times \\
& \times\left(\kappa_{1} \lambda-1\right)^{-1} \kappa_{1}^{-2}\left(\kappa_{1}-\kappa_{2}\right)^{-1}\left(\kappa_{1}-\kappa_{3}\right)^{-1} \kappa_{2}{ }^{-1} \kappa_{3}{ }^{-1}- \\
& -2 k_{2}{ }^{2} L_{0}{ }^{2} T\left(\mathrm{e}^{\frac{\left(\kappa_{2} \lambda-1\right) \theta}{\lambda}}-1\right)\left(\mathrm{e}^{-\frac{\theta}{\lambda}}-1\right)\left(1+\tau \kappa_{2}\right)\left(k_{1}+k_{2}\right) m^{-2} \tau^{-2} \times \\
& \times\left(\kappa_{2} \lambda-1\right)^{-1} \kappa_{2}^{-2}\left(\kappa_{1}-\kappa_{2}\right)^{-1}\left(\kappa_{2}-\kappa_{3}\right)^{-1} \kappa_{1}{ }^{-1} \kappa_{3}{ }^{-1}+ \\
& +2 k_{2}^{2} L_{0}^{2} T\left(\mathrm{e}^{\frac{\left(\kappa_{3} \lambda-1\right) \theta}{\lambda}}-1\right)\left(\mathrm{e}^{-\frac{\theta}{\lambda}}-1\right)\left(1+\tau \kappa_{3}\right)\left(k_{1}+k_{2}\right) m^{-2} \tau^{-2} \times \\
& \times\left(\kappa_{3} \lambda-1\right)^{-1} \kappa_{3}{ }^{-2}\left(\kappa_{1}-\kappa_{3}\right)^{-1}\left(\kappa_{2}-\kappa_{3}\right)^{-1} \kappa_{1}{ }^{-1} \kappa_{2}{ }^{-1}+ \\
& +k_{2}{ }^{2} L_{0}^{2} T\left(\mathrm{e}^{-\frac{\theta}{\lambda}}-1\right)^{2}\left(k_{1}+k_{2}\right) m^{-2} \tau^{-2} \kappa_{1}{ }^{-2} \kappa_{2}{ }^{-2} \kappa_{3}{ }^{-2}+ \\
& +k_{2}{ }^{2} L_{0}{ }^{2} T\left(\mathrm{e}^{\frac{\left(\kappa_{1} \lambda-1\right) \theta}{\lambda}}-1\right)^{2}\left(1+\tau \kappa_{1}\right)^{2}\left(m \kappa_{1}{ }^{2}+k_{1}+k_{2}\right) m^{-2} \tau^{-2} \times \\
& \times\left(\kappa_{1} \lambda-1\right)^{-2} \kappa_{1}^{-2}\left(\kappa_{1}-\kappa_{2}\right)^{-2}\left(\kappa_{1}-\kappa_{3}\right)^{-2}- \\
& -2 k_{2}^{2} L_{0}^{2} T\left(\mathrm{e}^{\frac{\left(\kappa_{2} \lambda-1\right) \theta}{\lambda}}-1\right)\left(\mathrm{e}^{\frac{\left(\kappa_{1} \lambda-1\right) \theta}{\lambda}}-1\right)\left(1+\tau \kappa_{2}\right)\left(1+\tau \kappa_{1}\right)\left(m \kappa_{2} \kappa_{1}+k_{1}+k_{2}\right) \times \\
& \times m^{-2} \tau^{-2}\left(\kappa_{2} \lambda-1\right)^{-1} \kappa_{2}^{-1} \kappa_{1}^{-1}\left(\kappa_{1} \lambda-1\right)^{-1}\left(\kappa_{1}-\kappa_{2}\right)^{-2}\left(\kappa_{2}-\kappa_{3}\right)^{-1}\left(\kappa_{1}-\kappa_{3}\right)^{-1}+ \\
& +2 k_{2}^{2} L_{0}^{2} T\left(\mathrm{e}^{\frac{\left(\kappa_{3} \lambda-1\right) \theta}{\lambda}}-1\right)\left(\mathrm{e}^{\frac{\left(\kappa_{1} \lambda-1\right) \theta}{\lambda}}-1\right)\left(1+\tau \kappa_{3}\right)\left(1+\tau \kappa_{1}\right)\left(m \kappa_{3} \kappa_{1}+k_{1}+k_{2}\right) \times \\
& \times m^{-2} \tau^{-2}\left(\kappa_{3} \lambda-1\right)^{-1} \kappa_{3}{ }^{-1} \kappa_{1}{ }^{-1}\left(\kappa_{1} \lambda-1\right)^{-1}\left(\kappa_{1}-\kappa_{3}\right)^{-2}\left(\kappa_{2}-\kappa_{3}\right)^{-1}\left(\kappa_{1}-\kappa_{2}\right)^{-1}+ \\
& +k_{2}{ }^{2} L_{0}{ }^{2} T\left(\mathrm{e}^{\frac{\left(\kappa_{2} \lambda-1\right) \theta}{\lambda}}-1\right)^{2}\left(1+\tau \kappa_{2}\right)^{2}\left(m \kappa_{2}{ }^{2}+k_{1}+k_{2}\right) m^{-2} \tau^{-2} \times \\
& \times\left(\kappa_{2} \lambda-1\right)^{-2} \kappa_{2}^{-2}\left(\kappa_{1}-\kappa_{2}\right)^{-2}\left(\kappa_{2}-\kappa_{3}\right)^{-2}- \\
& -2 k_{2}^{2} L_{0}^{2} T\left(\mathrm{e}^{\frac{\left(\kappa_{3} \lambda-1\right) \theta}{\lambda}}-1\right)\left(\mathrm{e}^{\frac{\left(\kappa_{2} \lambda-1\right) \theta}{\lambda}}-1\right)\left(1+\tau \kappa_{3}\right)\left(1+\tau \kappa_{2}\right)\left(m \kappa_{3} \kappa_{2}+k_{1}+k_{2}\right) \times \\
& \times m^{-2} \tau^{-2}\left(\kappa_{3} \lambda-1\right)^{-1} \kappa_{3}{ }^{-1} \kappa_{2}{ }^{-1}\left(\kappa_{2} \lambda-1\right)^{-1}\left(\kappa_{1}-\kappa_{3}\right)^{-1}\left(\kappa_{2}-\kappa_{3}\right)^{-2}\left(\kappa_{1}-\kappa_{2}\right)^{-1}+ \\
& +k_{2}{ }^{2} L_{0}{ }^{2} T\left(\mathrm{e}^{\frac{\left(\kappa_{3} \lambda-1\right) \theta}{\lambda}}-1\right)^{2}\left(1+\tau \kappa_{3}\right)^{2}\left(m \kappa_{3}{ }^{2}+k_{1}+k_{2}\right) m^{-2} \tau^{-2} \times \\
& \times\left(\kappa_{3} \lambda-1\right)^{-2} \kappa_{3}^{-2}\left(\kappa_{1}-\kappa_{3}\right)^{-2}\left(\kappa_{2}-\kappa_{3}\right)^{-2} \text {. }
\end{aligned}
$$


C

C.1

Representação de $x^{3}(t)$ na forma integral

$$
\begin{aligned}
x^{3}(t) & =\int_{0}^{\infty} \delta\left(t-t_{1}\right) x\left(t_{1}\right) d t_{1} \int_{0}^{\infty} \delta\left(t-t_{2}\right) x\left(t_{2}\right) d t_{2} \int_{0}^{\infty} \delta\left(t-t_{3}\right) x\left(t_{3}\right) d t_{3} \\
& =\lim _{\alpha \rightarrow 0^{+}} \int_{0}^{\infty} \int_{-\infty}^{\infty} \frac{d q_{1}}{2 \pi} e^{\left(i q_{1}+\alpha\right)\left(t-t_{1}\right)} x\left(t_{1}\right) d t_{1} \int_{0}^{\infty} \int_{-\infty}^{\infty} \frac{d q_{2}}{2 \pi} e^{\left(i q_{2}+\alpha\right)\left(t-t_{2}\right)} x\left(t_{2}\right) d t_{2} \times \\
& \times \int_{0}^{\infty} \int_{-\infty}^{\infty} \frac{d q_{3}}{2 \pi} e^{\left(i q_{3}+\alpha\right)\left(t-t_{3}\right)} x\left(t_{3}\right) d t_{3}, \\
& =\lim _{\alpha \rightarrow 0^{+}} \int_{-\infty}^{\infty} \frac{d q_{1}}{2 \pi} e^{\left(i q_{1}+\alpha\right) t} \tilde{x}\left(i q_{1}+\alpha\right) \times \int_{-\infty}^{\infty} \frac{d q_{2}}{2 \pi} e^{\left(i q_{2}+\alpha\right) t} \tilde{x}\left(i q_{2}+\alpha\right) \times \\
& \times \int_{-\infty}^{\infty} \frac{d q_{3}}{2 \pi} e^{\left(i q_{3}+\alpha\right) t} \tilde{x}\left(i q_{3}+\alpha\right), \\
& =\lim _{\alpha \rightarrow 0^{+}} \int_{-\infty}^{\infty} \int_{-\infty}^{\infty} \int_{-\infty}^{\infty} \frac{d q_{1}}{2 \pi} \frac{d q_{2}}{2 \pi} \frac{d q_{3}}{2 \pi} e^{\left(i q_{1}+i q_{2}+i q_{3}+3 \alpha\right) t} \times \\
& \times \tilde{x}\left(i q_{1}+\alpha\right) \tilde{x}\left(i q_{2}+\alpha\right) \tilde{x}\left(i q_{3}+\alpha\right) .
\end{aligned}
$$

\section{2}

\section{Transformada de Laplace para cada termo da Equação de Langevin}

$$
\begin{aligned}
\mathcal{L}\{m \dot{v}(t)\} & =s \tilde{v}(s)-v_{0} \\
\mathcal{L}\left\{-\int_{0}^{t} d t^{\prime} \phi\left(t-t^{\prime}\right) v\left(t^{\prime}\right)\right\} & =\tilde{\phi}(s) \tilde{v}(s) \\
\mathcal{L}\left\{-k_{1} x(t)-k_{2} x(t)\right\} & =-k_{1} \tilde{x}(s)-k_{2} \tilde{x}(s) \\
\mathcal{L}\left\{k_{2} L(t)\right\} & =\frac{k_{2}}{s(1+s \lambda)}, \\
\mathcal{L}\left\{-k_{3} x^{3}(t)\right\} & =-k_{3} \lim _{\alpha \rightarrow 0^{+}} \int_{-\infty}^{\infty} \int_{-\infty}^{\infty} \int_{-\infty}^{\infty} \frac{d q_{1}}{2 \pi} \frac{d q_{2}}{2 \pi} \frac{d q_{3}}{2 \pi} \times \\
& \times \frac{\tilde{x}\left(i q_{1}+\alpha\right) \tilde{x}\left(i q_{2}+\alpha\right) \tilde{x}\left(i q_{3}+\alpha\right)}{s-\left(i q_{1}+i q_{2}+i q_{3}+3 \alpha\right)}, \\
\tilde{v}(s) & =s \tilde{x}(s)-x_{0}, \\
\tilde{\phi}(s) & =\frac{\gamma T}{s \tau+1} .
\end{aligned}
$$




\section{C.3}

\section{Transformada de Laplace do segundo cumulante do ruído Gaussiano}

A seguir, vamos demonstrar os cálculos da transformada de Laplace do ruído térmico. Partindo, da expressão

podemos fazer

$$
\left\langle\eta(t) \eta\left(t^{\prime}\right)\right\rangle=\frac{D}{2 \tau} e^{\frac{-\left|t-t^{\prime}\right|}{\tau}}
$$

$$
\begin{aligned}
\left\langle\eta\left(s_{1}\right) \eta\left(s_{2}\right)\right\rangle & =\frac{D}{2 \tau}\left(\int_{0}^{\infty} \int_{0}^{t} e^{-\frac{\left(t-t^{\prime}\right)}{\tau}} e^{-s_{1} t} e^{-s_{2} t^{\prime}} d t^{\prime} d t+\int_{0}^{\infty} \int_{t}^{\infty} e^{-\frac{\left(t^{\prime}-t\right)}{\tau}} e^{-s_{1} t} e^{-s_{2} t^{\prime}} d t^{\prime} d t\right) \\
& =\frac{1}{s_{2}-1 / \tau}\left(\int_{0}^{\infty}\left[e^{-\left(s_{2}+1 / \tau\right) t}-e^{-\left(s_{1}+s_{2}\right) t}\right] d t\right)+\frac{1}{s_{2}+1 / \tau} \int_{0}^{\infty} e^{-\left(s_{1}+s_{2}\right) t} d t \\
& =\frac{D \tau}{2\left(s_{1} \tau+1\right)\left(s_{2} \tau+1\right)}+ \\
& +\frac{D}{\left(s_{1}+s_{2}\right)\left(s_{1} \tau+1\right)\left(s_{2} \tau+1\right)} .
\end{aligned}
$$

Quando $\tau \rightarrow$ 0, a Eq. (C-10) é reduzida ao ruído branco:

$$
\frac{D}{s_{1}+s_{2}}
$$

\section{C.4 \\ Comportamento dos $\kappa_{i}$}

A fim de entendermos a natureza da função de Green $g(t)$ e a função auxiliar $f(t)$, nós devemos estudar a natureza das raízes do numerador $R(s)$, as quais nós referirmos como $\kappa_{i}$, isto é

$$
m \tau\left(s-\kappa_{1}\right)\left(s-\kappa_{2}\right)\left(s-\kappa_{3}\right)=m \tau s^{3}+m s^{2}+\left(\Gamma+\tau \nu^{2}\right) s+\nu^{2},
$$

na qual $\Gamma=\gamma / m$ e $\nu^{2}=\left(k_{1}+k_{2}\right) / m$. Nós podemos utilizar o discriminante de $R(s)$, que nós referimos como $\Delta$, que é expresso a seguir:

$$
\Delta=\Gamma^{2}-4 \Gamma^{3} \tau-4 \nu^{2}+4 \Gamma \tau(5-3 \Gamma \tau) \nu^{2}-4 \tau^{2}(2+3 \Gamma \tau) \nu^{4}-4 \tau^{4} \nu^{6}
$$

Se nós tomarmos $\tau=0$, o discriminante se torna $\Delta=\Gamma^{2}-4 \nu^{2}$, que é o resultado usual para um sistema com ruído branco.

Podemos obter os valores de $\kappa_{i}$ exatamente através da solução para o polinômio do terceiro grau. Deste modo, temos as seguintes expressões para as raízes: 


$$
\begin{aligned}
& \kappa_{1}=-\frac{1}{3 \tau}\left\{1-\frac{2-6 \Gamma \tau-6 \Gamma^{2} \tau^{2}+2^{1 / 3}\left(-2+9 \Gamma \tau-18 \tau^{2} \nu^{2}+\sqrt{-27 \tau^{2} \Delta}\right)^{2 / 3}}{2^{2 / 3}\left(-2+9 \Gamma \tau-18 \tau^{2} \nu^{2}+\sqrt{-27 \tau^{2} \Delta}\right)^{1 / 3}}\right\} \\
& \kappa_{2}=-\frac{1}{3 \tau}\left\{1+\frac{2-6 \Gamma \tau-6 \Gamma^{2} \tau^{2}-(-2)^{1 / 3}\left(-2+9 \Gamma \tau-18 \tau^{2} \nu^{2}+\sqrt{-27 \tau^{2} \Delta}\right)^{2 / 3}}{2^{2 / 3}\left(2-9 \Gamma \tau+18 \tau^{2} \nu^{2}-\sqrt{-27 \tau^{2} \Delta}\right)^{1 / 3}}\right\} \quad(\mathrm{C}-14) \\
& \kappa_{3}=-\frac{1}{3 \tau}\left\{1-(-1)^{2 / 3} \frac{2-\Gamma \tau-6 \Gamma^{2} \tau^{2}+(-1)^{2 / 3}(2)^{1 / 3}\left(-2+9 \Gamma \tau-18 \tau^{2} \nu^{2}+\sqrt{-27 \tau^{2} \Delta}\right)^{2 / 3}}{2^{2 / 3}\left(-2+9 \Gamma \tau-18 \tau^{2} \nu^{2}+\sqrt{-27 \tau^{2} \Delta}\right)^{1 / 3}}\right\},
\end{aligned}
$$

na qual nós utilizamos $\Delta$ como discriminante para simplificar. A dependência entre as variáveis $(\Gamma, \nu$ e $\tau$ ) é extremamente não trivial e não é muito claro os regimes que podemos obter pelas soluções. Nós demonstramos alguns valores típicos para alguns exemplos na Figura (C.1).
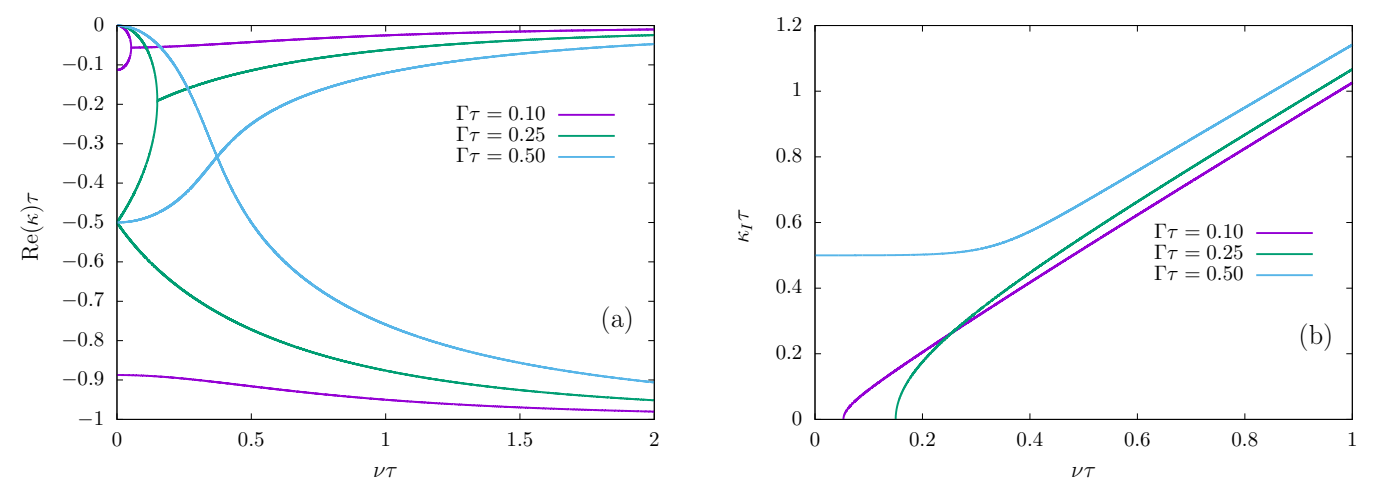

Figura C.1: Comportamento da parte real das raízes $\kappa$ (a) e parte imaginária das raízes $\kappa\left(\kappa_{I}\right)$ (b) como uma função de $\nu$ para diferentes valores de $\Gamma$ (na escala de $\tau$ ). Note que a parte real é sempre negativa e que $\kappa_{I}$ cresce para os valores de $\nu$ suficientemente grandes.

Apesar da complexidade citada anteriormente, algumas informações gerais podem ser extraídas das raízes. A componente real dos $\kappa$ s sempre serão negativas, indicando que as soluções sempre se aproximarão de um limite e não divergirão. Dado que todos os coeficientes do polinômio são reais e positivos, o sinal do discriminante determinará a natureza das raízes. Estamos interessados em determinar os casos em que todas as raízes sã reais $(\Delta \geq 0)$ e o caso com oscilações, no qual duas raízes são o complexo conjugado uma da outra $(\Delta<0)$. Com este objetivo, nós criamos umaa ilustração que engloba todos os possíveis sinais de $\Delta$, reduzindo a dois parâmetros: escolhendo $\tau$ como a escala de tempo e utilizando os parâmetros adimensionais $\nu \tau$ e $\Gamma \tau$ ou escolhendo $1 / \nu$ como a escala de tempo e utilizando os parâmetros adimensionais $\nu \tau$ e $\Gamma / \nu$. Os dois casos são mostrados na Figura (C.2). 

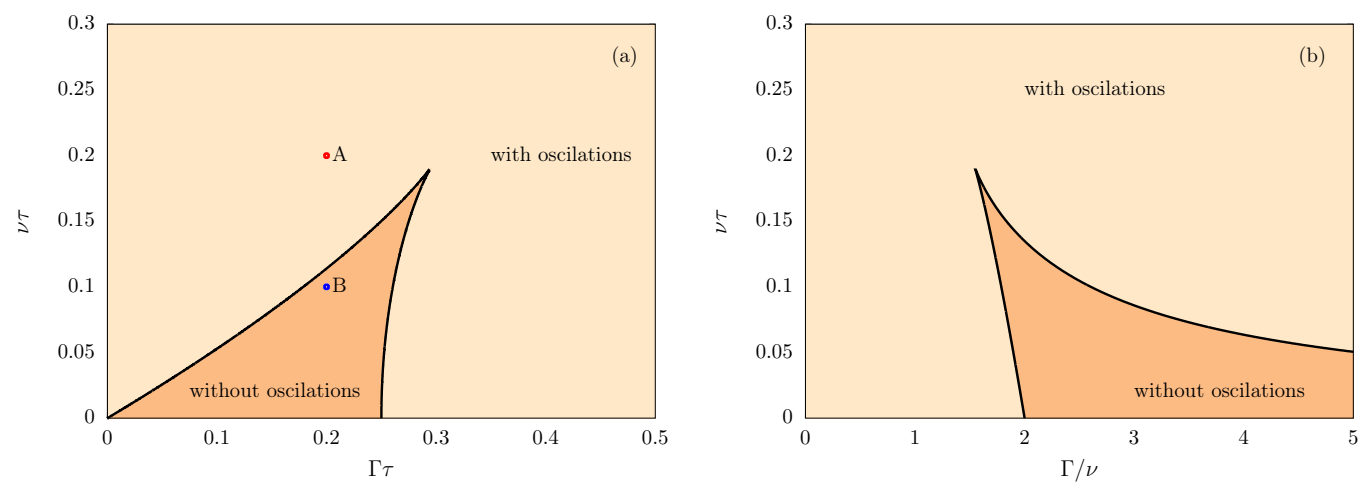

Figura C.2: Destacamos os valores dos parâmetros do sistema para os quais as oscilações podem ser observadas. Para um valor suficientemente grande de $\tau$, o discriminante sempre será negativo, daí o sistema apresentará oscilações.

Podemos escrever a função de Green, com o discriminante positivo, da seguinte forma:

$$
\begin{aligned}
g(t) & =\frac{\left(\kappa_{1} \tau-1\right) e^{-\kappa_{1} t}}{m \tau\left(\kappa_{1}-\kappa_{2}\right)\left(\kappa_{1}-\kappa_{3}\right)}+\frac{\left(\kappa_{2} \tau-1\right) e^{-\kappa_{2} t}}{m \tau\left(\kappa_{2}-\kappa_{1}\right)\left(\kappa_{2}-\kappa_{3}\right)}+ \\
& +\frac{\left(\kappa_{3} \tau-1\right) e^{-\kappa_{3} t}}{m \tau\left(\kappa_{3}-\kappa_{1}\right)\left(\kappa_{3}-\kappa_{2}\right)}
\end{aligned}
$$

Já para o discriminante negativo $\left(\kappa_{2,3}=\kappa_{R}+ \pm \mathrm{i} \kappa_{I}\right)$ surgem oscilações com frequência $\operatorname{Im}\left(\kappa_{2}\right)=\operatorname{Im}\left(\kappa_{3}\right)=\kappa_{I}$, de modo que

$$
\begin{aligned}
g(t) & =\frac{\left(1-\kappa_{1} \tau\right) e^{-\kappa_{1} t}}{m \tau\left(\kappa_{I}^{2}+\left(\kappa_{1}-\kappa_{R}\right)^{2}\right)}+\frac{\left(\kappa_{1} \tau-1\right) e^{-\kappa_{R} t}}{m \tau\left(\kappa_{I}^{2}+\left(\kappa_{1}-\kappa_{R}\right)^{2}\right)} \cos \left(\kappa_{I} t\right)+(\mathrm{C}-16) \\
& +\frac{\left(\kappa_{1}-\kappa_{R}-\kappa_{1} \kappa_{R} \tau+\left(\kappa_{I}^{2}+\kappa_{R}^{2}\right) \tau\right) e^{-\kappa_{R} t}}{m \tau\left(\kappa_{I}^{2}+\left(\kappa_{1}-\kappa_{R}\right)^{2}\right)} \operatorname{sen}\left(\kappa_{I} t\right) .
\end{aligned}
$$

A função auxiliar para o discriminante positivo é

$$
\begin{aligned}
f(t) & =\frac{\left(\kappa_{1}^{2} \tau-\kappa_{1}+\Gamma\right) e^{-\kappa_{1} t}}{m \tau\left(\kappa_{1}-\kappa_{2}\right)\left(\kappa_{1}-\kappa_{3}\right)}+\frac{\left(\kappa_{2}^{2} \tau-\kappa_{2}+\Gamma\right) e^{-\kappa_{2} t}}{m \tau\left(\kappa_{2}-\kappa_{1}\right)\left(\kappa_{2}-\kappa_{3}\right)}+ \\
& +\frac{\left(\kappa_{3}^{2} \tau-\kappa_{3}+\Gamma\right) e^{-\kappa_{3} t}}{m \tau\left(\kappa_{3}-\kappa_{1}\right)\left(\kappa_{3}-\kappa_{2}\right)}
\end{aligned}
$$

e para o discriminante negativo 


$$
\begin{aligned}
f(t) & =\frac{\kappa_{1}^{2} \tau-\kappa_{1}+\Gamma}{m \tau\left(\kappa_{1}-\kappa_{2}\right)\left(\kappa_{1}-\kappa_{3}\right)} e^{-\kappa_{1} t}+\frac{\left(\Gamma+\kappa_{1}+\left(\kappa_{I}+\kappa_{R}\left(\kappa_{T}-2 \kappa_{1}\right) \tau\right)\right) e^{-\kappa_{R} t}}{m \tau\left(\kappa_{I}^{2}+\left(\kappa_{1}-\kappa_{R}\right)^{2}\right)} \cos \left(\kappa_{I} t\right)+ \\
& +\frac{\left(\Gamma+\kappa_{1}+\left(\kappa_{I}^{2}+\kappa_{R}\left(\kappa_{R}-2 \kappa_{1}\right)\right) \tau\right) e^{-\kappa_{R} t}}{m \tau\left(\kappa_{I}^{2}+\left(\kappa_{1}-\kappa_{R}\right)^{2}\right)} \operatorname{sen}\left(\kappa_{I} t\right) .
\end{aligned}
$$

Na Figura C.3 nós mostramos ambos os regimes.

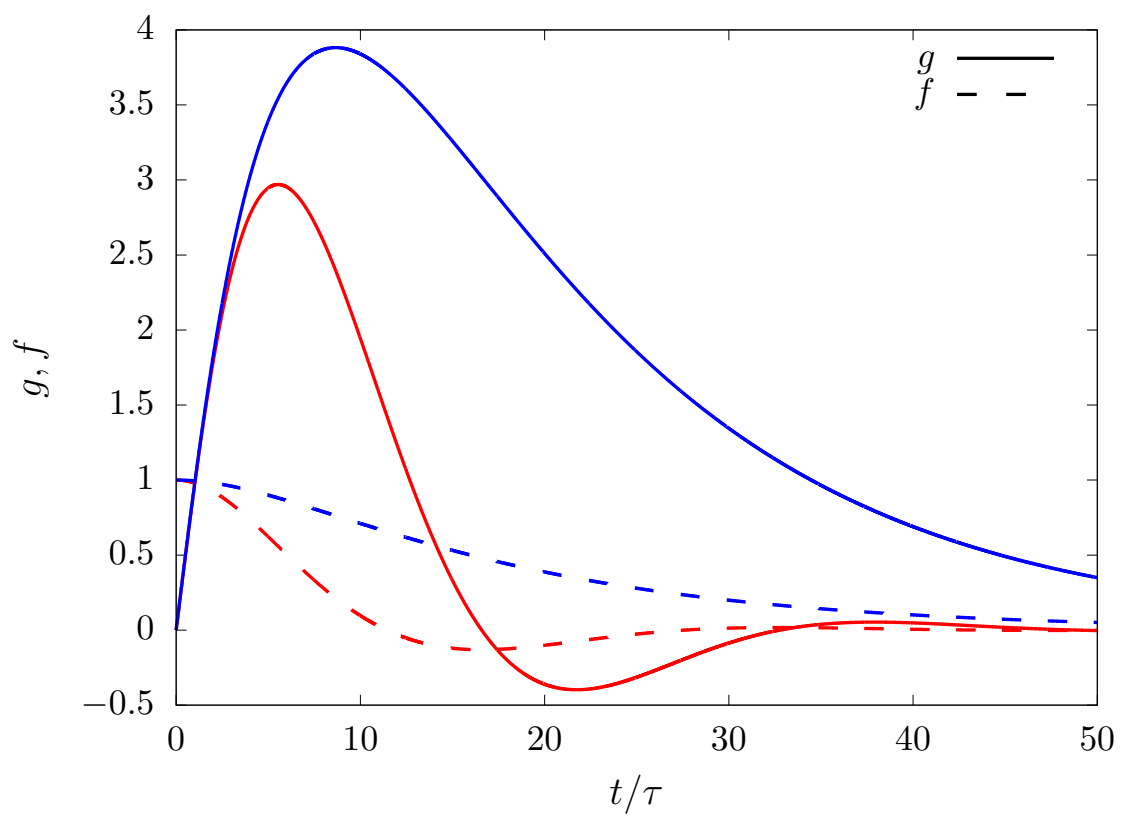

Figura C.3: Destacamos o comportamento da função de Green $g(t)$ e a função auxiliar $f(t)$ para os pontos A e B da Figura C.2. Quando o discriminante é positivo (linhas azuis), as funções sempre decaem. Quando os discriminante é negativo (linhas vermelhas), as funções apresentam um comportamento oscilatório. 